\title{
Measurement of the $W$ Boson Mass at the Collider Detector at Fermilab from a Fit to the Transverse Momentum Spectrum of the Muon.
}

by

\author{
Ian E. Vollrath
}

A thesis submitted in conformity with the requirements

for the degree of Doctor of Philosophy

Graduate Department of Physics

University of Toronto

Copyright (C) 2007 by Ian E. Vollrath 


\begin{abstract}
Measurement of the $W$ Boson Mass at the Collider Detector at Fermilab from a Fit to the Transverse Momentum Spectrum of the Muon.

\author{
Ian E. Vollrath \\ Doctor of Philosophy \\ Graduate Department of Physics \\ University of Toronto
}

2007

This thesis describes a measurement of the $W$ boson mass from a fit to the transverse momentum spectrum of the muon in $W$ decay. In past measurements this technique was used as a cross-check, however, now presents the best method in terms of systematic uncertainty. We discuss all sources of systematic uncertainty with emphasis on those to which the muon $p_{T}$ measurement is particularly sensitive, specifically, those associated with modelling the production and decay of $W$ bosons. The data were collected with the CDF II detector between March 2002 and September 2003 and correspond to an integrated luminosity of $(191 \pm 11) \mathrm{pb}^{-1}$. We measure the $W$ mass to be $(80.316 \pm$ $\left.0.066_{\text {stat. }} \pm 0.051_{\text {syst. }}\right) \mathrm{GeV} / c^{2}=(80.316 \pm 0.083) \mathrm{GeV} / c^{2}$.
\end{abstract}




\section{Dedication}

This thesis is dedicated to my parents. 


\section{Acknowledgements}

I would like to thank very much my supervisor William Trischuk for his support and guidance.

I would also like to thank the other members of the $W$ mass group: Oliver Stelzer-Chilton, Ashutosh Kotwal, Chris Hays, and Larry Nodulman.

Many thanks to my family who have continuously encouraged me to pursue my interests to the fullest.

Thanks to all my friends who have made my time as a graduate student enjoyable. 


\section{Contents}

1 Introduction $\quad 1$

2 Theoretical Background $\quad 5$

$2.1 W$ Boson Production at the Tevatron . . . . . . . . . . . . 10

2.2 Measuring $M_{W} \ldots \ldots \ldots \ldots \ldots \ldots$

3 The CDF II Detector and the Fermilab Tevatron $\quad 18$

3.1 The Tevatron Accelerator Chain . . . . . . . . . . . . . 18

3.1.1 Proton Production and Acceleration . . . . . . . . . . 18

3.1.2 Antiproton Production and Acceleration . . . . . . . . . . 19

3.1.3 The Tevatron ...................... 19

3.2 The CDF II Detector . . . . . . . . . . . . . . 21

3.2.1 The CDF Coordinate System and Transverse Quantities . . . . 21

3.2.2 Charged Particle Tracking Systems . . . . . . . . . . . 23

3.2 .3 Calorimeter Systems _. . . . . . . . . . . . . 26

3.2 .4 Muon Chambers ................... 28

3.2.5 Cherenkov Luminosity Counter . . . . . . . . . . . 31

3.2.6 Data Acquisition System . . . . . . . . . . . . 31

4 Event Generation $\quad 34$

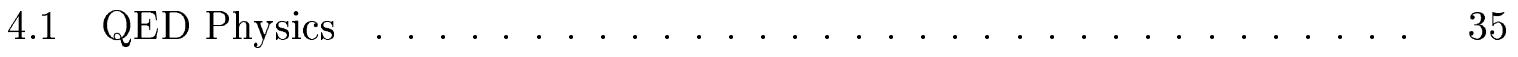


4.1 .1 QED with WGRAD $\ldots \ldots \ldots \ldots \ldots \ldots \ldots$

4.1 .2 Mass Shifts . . . . . . . . . . . . . . . 37

4.1.3 Including QED Effects in the Fit Templates . . . . . . . . . 42

4.1 .4 Second Photon . . . . . . . . . . . . . . . 45

$4.1 .5 \quad$ QED Radiation for $Z \rightarrow l l \ldots \ldots \ldots \ldots \ldots \ldots$

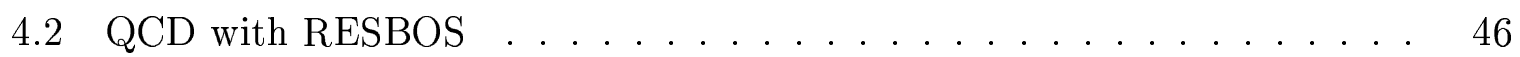

$4.2 .1 \quad W$ Boson Generation . . . . . . . . . . . . . . . . 48

$4.2 .2 \quad Z$ Boson Generation . . . . . . . . . . . . . 48

4.2.3 Impact of QCD Processes on the $W$ Mass $\ldots \ldots \ldots \ldots \ldots$

4.2.4 Systematic Uncertainties from RESBOS . . . . . . . . . . 52

4.3 QED/QCD Correlations . . . . . . . . . . . . . . 53

4.4 Parton Distribution Functions . . . . . . . . . . . . . . . . 54

5 Event Selection and Backgrounds $\quad 57$

5.1 Event Selection . . . . . . . . . . . . . . . . . . 57

$5.1 .1 \quad J / \psi \rightarrow \mu \mu$ Data Sample . . . . . . . . . . . . 58

$5.1 .2 \Upsilon \rightarrow \mu \mu$ Data Sample. . . . . . . . . . . . . . . 59

5.1.3 High $p_{T}$ Muon Samples: $W \rightarrow \mu \nu$ and $Z \rightarrow \mu \mu \ldots \ldots \ldots$

5.1 .4 Selection Efficiencies $\ldots \ldots \ldots \ldots$. . . . . . . . 62

5.2 Backgrounds . . . . . . . . . . . . . . . . 63

$5.2 .1 \quad Z \rightarrow \mu \mu$ and $W \rightarrow \tau \nu \ldots \ldots \ldots \ldots \ldots \ldots \ldots$

5.2 .2 Kaon Background . . . . . . . . . . . . . . . 64

5.2 .3 Hadronic Jets . . . . . . . . . . . . . . . . . . 66

5.2 .4 Cosmic Background . . . . . . . . . . . . . . 67

5.2 .5 Combined Background $\ldots \ldots \ldots \ldots \ldots$

6 Fast Detector Simulation $\quad 70$

6.1 Material Modelling . . . . . . . . . . . . . . . . . . . 71 
6.2 COT Simulation . . . . . . . . . . . . . . . . 71

6.3 Muon Acceptance Modelling . . . . . . . . . . . . . . . . . . 74

6.4 Hadronic Recoil . . . . . . . . . . . . . . . . . . . . . 74

6.4.1 Muon Removal Correction . . . . . . . . . . . . . 76

6.4.2 Determining the Recoil Model Parameters . . . . . . . . . . 77

$\begin{array}{llr}7 & \text { Detector Calibration } & 81\end{array}$

7.1 COT Momentum Scale from $J / \psi \rightarrow \mu \mu \ldots \ldots \ldots \ldots$. . . . 82

7.1 .1 Fitting templates . . . . . . . . . . . . . 82

$7.1 .2 \quad J / \psi$ Analysis $\ldots \ldots \ldots \ldots \ldots \ldots \ldots$

7.1.3 Systematic Uncertainties on the $J / \psi$ Scale . . . . . . . . 86

7.2 COT Momentum Scale from $\Upsilon(1 S) \rightarrow \mu \mu \ldots \ldots \ldots$

7.2 .1 Fitting templates $\ldots \ldots \ldots \ldots \ldots \ldots$

$7.2 .2 \Upsilon$ Analysis . . . . . . . . . . . . . . . . 89

7.2.3 Systematic Uncertainties on the $\Upsilon$ Scale . . . . . . . . . 91

7.3 Combined Momentum Scale . . . . . . . . . . . . . . . . . . 91

7.4 Momentum Scale Cross-check with $Z \rightarrow \mu \mu \ldots \ldots \ldots \ldots$

$\begin{array}{llr}8 & \text { Results } & 95\end{array}$

8.1 Fitting Data to Monte Carlo . . . . . . . . . . . . . . . . 95

8.2 Correlations between Mass Fits . . . . . . . . . . . . . 96

8.3 Systematic Uncertainties on the $p_{T}$ Fit $\ldots \ldots \ldots \ldots$

8.4 Result . . . . . . . . . . . . . . . . . . . 99

8.5 Cross-checks . . . . . . . . . . . . . . . . . . 100

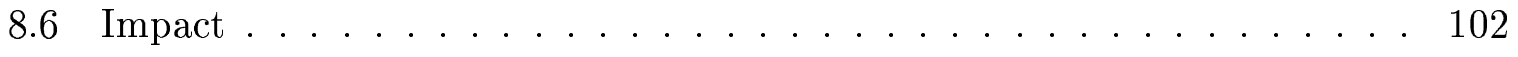

8.7 Conclusion . . . . . . . . . . . . . . . . . . . . . 104

$\begin{array}{ll}\text { Bibliography } & 104\end{array}$ 


\section{List of Tables}

2.1 The particles of the Standard Model. . . . . . . . . . . . 5

2.2 Masses of Standard Model particles. . . . . . . . . . . . . 6

4.1 Shifts in the $W$ mass due to various QED processes. . . . . . . . . . 39

4.2 Systematic uncertainties due to $\delta_{s} \ldots \ldots \ldots \ldots$. . . . . . . 42

4.3 Shifts in the $W$ mass due to sampling a histogram that contains WGRAD final state photons. . . . . . . . . . . . . . 45

4.4 Shifts in the fitted $Z$ mass for LO and FSR via sampling a $Q^{2}$-scaled WGRAD histogram. . . . . . . . . . . . . 46

4.5 Summary of QED and fitter systematic uncertainties for the mass fit in the muon and electron channels. . . . . . . . . . . . . 47

4.6 Normalization for the additional contributions to the $Z$ lineshape of the $\gamma$-pole and $Z / \gamma$ interference. . . . . . . . . . . . 51

4.7 Systematic uncertainties due to varying the $g$ parameters in RESBOS about their nominal values. . . . . . . . . . . . . 54

$4.8 Z$ mass shifts from sampling a boosted QED histogram. . . . . . . . 55

5.1 Data samples used in the $W$ mass analysis. . . . . . . . . . 58

7.1 Systematic uncertainties on the $J / \psi \rightarrow \mu \mu$ momentum scale $\ldots . . . \quad 88$

7.2 Systematic uncertainties on the $\Upsilon(1 S) \rightarrow \mu \mu$ momentum scale . . . . . 92

8.1 Measured correlations between $W$ mass fit types in the muon channel. . . 97 
8.2 Systematic uncertainties on the $W$ mass for the $p_{T}^{\mu}$ method. . . . . . . 98

8.3 Cross-checks of the $W$ mass measurement. . . . . . . . . . . . . 102

8.4 Current world's best $W$ mass measurements. . . . . . . . . . . . . . 103 


\section{List of Figures}

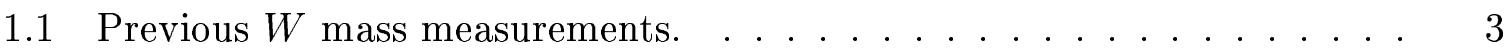

2.1 First order radiative corrections to the $W$ propagator. . . . . . . . 8

2.2 Higgs mass constraint from $M_{W}$ and $M_{\text {top }} \ldots \ldots \ldots \ldots \ldots$

2.3 Expected Higgs mass from $\chi^{2}$ minimization of Standard Model fits. . . . 9

2.4 Leading order production and decay of a $W$ boson at the Tevatron. . . . 10

2.5 Higher order processes contributing to $W$ boson production. . . . . . . . 11

2.6 Cartoon of $W$ boson decay illustrating the hadronic recoil. . . . . . . 15

2.7 Sensitivities of the transverse momentum and transverse mass fits for the $W$ mass. . . . . . . . . . . . . . . . . . . 17

3.1 Fermilab accelerator complex. . . . . . . . . . . . . . . . . 20

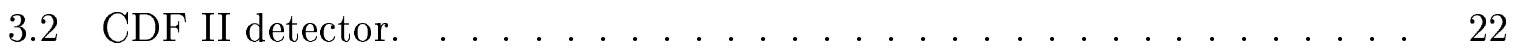

3.3 Tracking systems at CDF. . . . . . . . . . . . . . . 23

3.4 Portion of the COT illustrating the arrangement of superlayers. . . . . 25

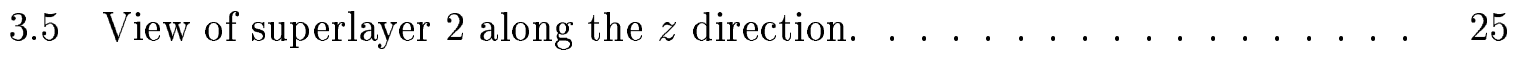

3.6 A wedge of the central calorimeter. . . . . . . . . . . . . 27

3.7 Schematic drawing of the muon detectors located behind the central calorimeter. .............................. 29

3.8 Muon chamber in the CMU. . . . . . . . . . . . . . . . . . 30

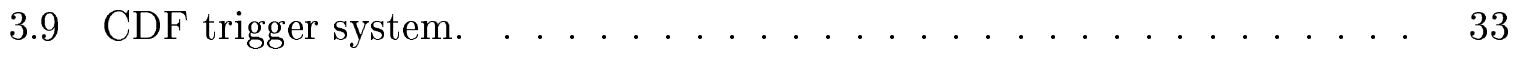


4.1 Effect of $\mathcal{O}(\alpha)$ electroweak corrections on $M_{T}^{W}$ and $p_{T}^{l}$ distributions. . . . 38

4.2 Shifts in the $W$ mass as a function of $\delta_{s} \ldots \ldots \ldots$. . . . . . . 41

4.3 Projection of $\sqrt[3]{y}$ for muons and electrons. . . . . . . . . . . . . 43

4.4 Projection of $\sqrt{\Delta R}$ for muons and electrons. . . . . . . . . . . . 44

4.5 Helicity amplitudes from RESBOS and DYRAD. . . . . . . . . . 49

4.6 Drell-Yan and $Z / \gamma$ interference contributions to $Z$ production from RES-

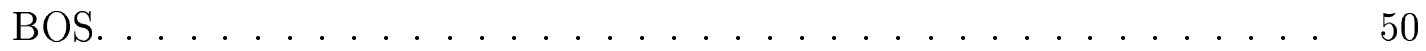

4.7 Comparison of NLO QCD with NLO QCD plus resummation in the $M_{T}^{W}$

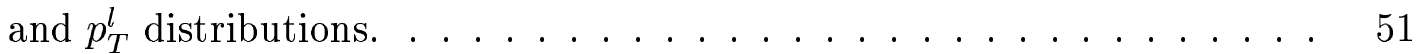

$4.8 W p_{T}$ spectrum generated by RESBOS for various $g_{2}$ values. . . . . . 53

4.9 Changes in $M_{W}$ for 20 error PDFs. . . . . . . . . . . 56

5.1 Muon identification efficiency as a function of $u_{\|} \ldots \ldots 2$

$5.2 t_{0}$ distributions of cosmics and $W \rightarrow \mu \nu$ in the $d_{0}$ sideband region. . . . 65

5.3 Calorimeter isolation for $W \rightarrow \mu \nu$ events and $Z \rightarrow \mu \mu$ with QCD added. 66

$5.4 \quad \mathbb{H}_{T}$ distribution for $W \rightarrow \mu \nu$ data and $W$ Monte Carlo with QCD added. 67

$5.5 \quad t_{0}$ distributions of $W$ data, $Z$ data, and cosmic ray muons. . . . . . . 68

5.6 The $p_{T}$ spectra of all the backgrounds to $W \rightarrow \mu \nu . \ldots \ldots$. . . . . 69

6.1 Double Gaussian multiple scattering model fit to MUSCAT data. . . . . 73

6.2 Schematic diagram showing $u_{\|}$and $u_{\perp}$ directions. . . . . . . 75

6.3 Mean EM and hadronic energy in each tower surrounding the muon track. 76

6.4 Definition of the $\eta-\xi$ coordinate system used for the recoil model. . . . 77

6.5 Mean and RMS $p_{T}^{\eta}$-balance plots for $Z \rightarrow \mu \mu$. . . . . . . . 78

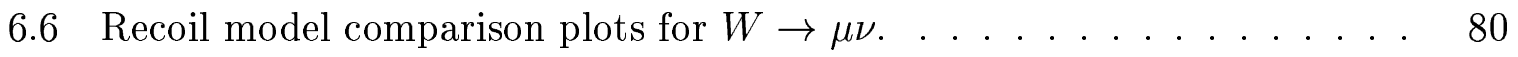

7.1 Di-muon $p_{T}$ and sum curvature distributions after tuning PYTHIA. . . . 83

7.2 Invariant mass of $J / \psi \rightarrow \mu \mu \ldots \ldots \ldots \ldots$

7.3 $\Delta p / p$ vs. $\Delta \cot \theta$ between the $\mu^{+}$and $\mu^{-}$in $J / \psi$ decay. . . . . . . 85 
$7.4 \Delta p / p$ vs. mean curvature for $J / \psi$ events after material scaling. . . . . . 86

$7.5 \Delta p / p$ vs. $\cot \theta$ before corrections. . . . . . . . . . 87

$7.6 \Delta p / p$ vs. muon $\phi \ldots \ldots \ldots \ldots \ldots \ldots \ldots \ldots$

7.7 Upsilon $1 S, 2 S$, and $3 S$ resonances. . . . . . . . . . . . 90

7.8 Momentum scales obtained from $\Upsilon(1 S) \rightarrow \mu \mu$ events. . . . . . . . . . 90

$7.9 Z \rightarrow \mu \mu$ invariant mass fit. . . . . . . . . . . . 94

8.1 Illustration of the $p_{T}^{\mu}$ templates generated by the fast simulation. . . . . 96

8.2 Correlations between $W$ mass fit types. . . . . . . . . . . . . . 97

$8.3 W$ mass from a fit to the $p_{T}^{\mu}$ spectrum. . . . . . . . . . . 100

8.4 The $\log$ likelihood of the fit to the $p_{T}^{\mu}$ spectrum. . . . . . . . . 101

8.5 Signed $\chi$ plot of the fit to the $p_{T}^{\mu}$ spectrum. . . . . . . . . . . . 101 


\section{Chapter 1}

\section{Introduction}

One of the greatest scientific achievements in human history was the realization that the world around us is made from a relatively small number of fundamental constituents. This was perhaps first encapsulated in Mendeleev's periodic table of the elements, which allowed us to write down on one page the material that made up stars and nebulae, to sand and viruses. This achievement satisfied a profound desire of human beings to find simple structure in a chaotic world. However, this idea was not new; the ancient Greeks believed everything was made of four simple elements: earth, air, water, and fire. The fundamental approach has not changed for over two thousand years, the only difference is that we now have a different model and have tested it extensively, so we believe it is a more accurate description of the world. This model, which is called the Standard Model of particle physics [1], is analogous to the periodic table, except that it applies to the fundamental constituents of matter, things like electrons and photons, and more esoteric objects like quarks and Higgs bosons. Not only does it describe matter at the most fundamental level, it describes the interactions of that matter, based on three of the four known fundamental forces; the strong, weak, and electromagnetic forces. Unfortunately, we know the model is incomplete since the fourth fundamental force, gravity, has never successfully been incorporated [1]. 
The Standard Model consists of point-like fundamental particles, quarks and leptons, that are subject to the strong, weak, and electromagnetic forces. These forces do not manifest themselves as fields, rather, their impact is felt by the exchange of other pointlike particles, the so-called force carriers. These are the $W$ and $Z$ bosons for the weak force, gluons for the strong force, and the photon for the electromagnetic force. These particles, like all others, are characterized by certain fundamental properties such as mass, spin, and electric charge, among others. The topic of this thesis is the measurement of the mass of the $W$ boson, one of the mediators of the weak force. One may ask why we are interested in measuring the mass of this particular particle? Furthermore, since the mass has already been precisely measured many times before, why do it again? The reason is that a measurement of the $W$ mass, combined with the measurement of the mass of one of the quarks in the Standard Model (the top quark) constrains the mass of the as yet undiscovered Higgs boson. The Higgs boson is the last missing particle of the Standard Model that must exist for the model to work, yet it so far has eluded experimental detection. The Higgs boson is an artifact of a broken symmetry in the model that is responsible for generating particle masses. The importance of discovering the Higgs cannot be overstated, as its discovery will fill in one of the remaining gaps in the Standard Model and will usher in a new era in particle physics.

The primary method by which we investigate the nature of fundamental particles and their interactions is by colliding particles together at high energies and observing what comes out of these collisions, using particle detectors. The data used in this thesis were collected using the CDF II detector at the Fermilab Tevatron. Currently, the Fermilab Tevatron is the highest energy particle collider in the world and is unique in its ability to investigate physics at the energy frontier. Unfortunately, it appears as though direct observation of the Higgs boson is just beyond the reach of the Tevatron, so the best we can do right now is try to pinpoint what mass it might have based on other measured quantities. We believe the Higgs will be discovered in the next few years by the Large 
Hadron Collider (LHC) at CERN, which will provide the highest energy collisions in the world (more than seven times that of the Tevatron). The measurement of the $W$ mass will still be an important endeavour once we have discovered the Higgs and measured its mass, as it will then provide an incisive test of the Standard Model.

The $W$ boson was discovered along with the $Z$ boson in 1982 at the UA1/2 experiments at CERN [2]. Since then its mass has been measured numerous times. Some recent results are shown in Figure 1.1. The current world average value is $(80.392 \pm 0.029)$ $\mathrm{GeV} / c^{2}[3]$.

\section{W-Boson Mass [GeV]}

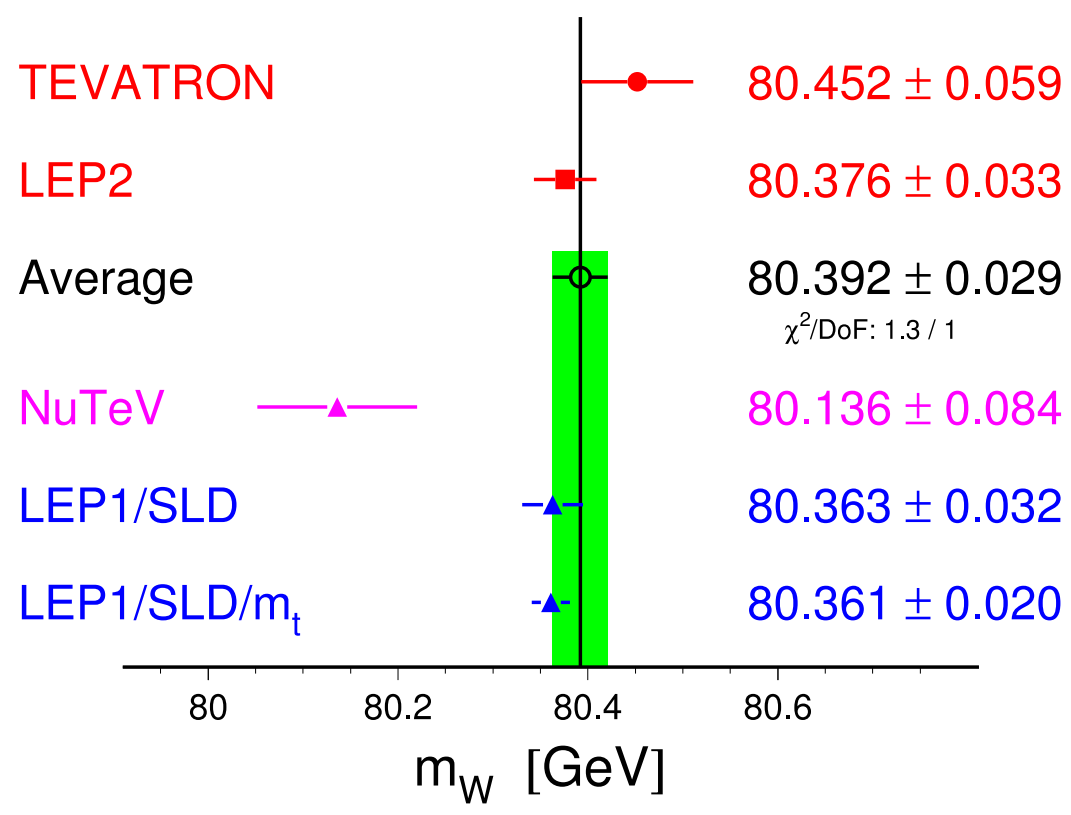

Figure 1.1: Previous $W$ mass measurements [3]. Direct measurements come from the Tevatron and LEP2, indirect measurements come from LEP1, SLD, and NuTeV.

We measure the mass by looking at the decay products of the $W$ boson, with the decays to an electron and a neutrino and a muon and a neutrino providing the only viable measurement channels. Furthermore, since we cannot reconstruct the $W$ mass directly, we resort to extracting the mass from fits to various kinematic quantities of the $W$ boson, charged lepton, or neutrino; for example, the transverse momentum spectrum of the muon 
(where transverse momentum is the momentum of a particle perpendicular to the beam direction in the Tevatron collider). Since this is a precision measurement, we devote considerable effort to the evaluation of the systematic uncertainties on the measured value. It is particularly important to thoroughly understand these uncertainties now, since in the near future the statistical uncertainty on the measurement will decrease dramatically. For example, by 2009 the CDF II detector will have collected a total data sample of $W$ bosons such that the statistical uncertainty on the muon transverse momentum measurement will be on the order of $10 \mathrm{MeV} / c^{2}$, approximately a factor of five smaller than the current systematic uncertainty.

The measurement of the $W$ mass is an ongoing project that will not end with the completion of this thesis. Future measurements will be valuable to further constrain the Higgs mass or cross-check the Standard Model. A precise measurement of the $W$ mass requires an intimate understanding of many important effects both experimental, such as detector response and resolution, and theoretical, such as higher order quantum corrections. Therefore the measurement provides ancillary benefits to other measurements in which similar processes occur and for that reason will probably be repeated time and again for years to come.

The thesis is laid out as follows: Chapter 2 presents the aspects of the Standard Model of particle physics relevant to the $W$ boson. In Chapter 3 the experimental apparatus is discussed: namely the Fermilab Tevatron and the CDF II detector. Chapter 4 describes the methods we use to generate Monte Carlo samples for fitting the data. Chapter 5 outlines the event selection criteria for obtaining the data samples and the backgrounds present in $W \rightarrow \mu \nu$ sample. We describe the detector simulation and calibration in Chapters 6 and 7, respectively. The final results are presented in Chapter 8. 


\section{Chapter 2}

\section{Theoretical Background}

Currently all matter and forces, except gravity, are described by the Standard Model. Although inherently incomplete, it has described particle interactions with great accuracy for the past 40 years.

The Standard Model is a collection of related theories; the electroweak theory $[4,5]$, which unifies the electromagnetic force and the weak force, and Quantum ChromoDynamics (QCD) [6], the theory describing the interactions of the quarks. In the Standard Model, all matter consists of fermions which interact via the exchange of gauge bosons, see Table 2.1 .

\begin{tabular}{|c|c|c|c|c|}
\hline \multicolumn{4}{|c|}{ Fermions } & Bosons \\
\hline Quarks & Charge (e) & Leptons & Charge (e) & $W^{ \pm}, Z^{0}, \gamma$ \\
\hline $\begin{array}{lll}u & c & t\end{array}$ & $+2 / 3$ & $e \quad \mu \quad \tau$ & -1 & 8 gluons, \\
\hline$d \quad s \quad b$ & $-1 / 3$ & $\begin{array}{lll}\nu_{e} & \nu_{\mu} & \nu_{\tau}\end{array}$ & 0 & Higgs $(h)$ \\
\hline
\end{tabular}

Table 2.1: The particles of the Standard Model. The fermions have spin 1/2 while the bosons have spin 1, except the Higgs, which is spin 0. Each fermion has an antiparticle. The quarks and gluons carry colour charges. The Higgs boson has yet to be discovered.

The fermions in the Standard Model are arranged in three generations; the first 
generation consists of the up $(u)$ and down $(d)$ quarks and the electron $(e)$ and electron neutrino $\left(\nu_{e}\right)$, the second generation consists of the charm $(c)$ and strange $(s)$ quarks and the muon $(\mu)$ and muon neutrino $\left(\nu_{\mu}\right)$, the third generation consists of the top $(t)$ and bottom $(b)$ quarks and the tau $(\tau)$ and tau neutrino $\left(\nu_{\tau}\right)$. The fermion masses are increasingly larger for each generation, see Table 2.2. It is not known why there are three generations of particles.

\begin{tabular}{|c|c|c|c|c||c|c|}
\hline Generation & Quarks & $\begin{array}{c}\text { Mass } \\
\left(\mathrm{MeV} / c^{2}\right)\end{array}$ & Leptons & $\begin{array}{c}\text { Mass } \\
\left(\mathrm{MeV} / c^{2}\right)\end{array}$ & Bosons & $\begin{array}{c}\text { Mass } \\
\left(\mathrm{MeV} / c^{2}\right)\end{array}$ \\
\hline $1^{\text {st }}$ & $u$ & 1.5 to 4 & $e$ & 0.511 & $W^{ \pm}$ & $80392 \pm 29$ \\
& $d$ & 4 to 8 & $\nu_{e}$ & $<0.000003$ & $Z^{0}$ & $91188 \pm 2$ \\
\cline { 1 - 3 } $2^{\text {nd }}$ & $c$ & 1150 to 1350 & $\mu$ & 106 & $\gamma$ & 0 \\
& $s$ & 80 to 130 & $\nu_{\mu}$ & $<0.19$ & gluons & 0 \\
\hline \multirow{2}{*}{$3^{\text {rd }}$} & $t$ & $171400 \pm 2100$ & $\tau$ & 1777 & & \\
& $b$ & 4100 to 4400 & $\nu_{\tau}$ & $<18.2$ & & \\
\hline
\end{tabular}

Table 2.2: Masses of Standard Model particles [7,3]. The masses of all the quarks except the top are not well known, so only ranges are given. The masses of the charged leptons are exact for the number of significant figures shown. Only limits have been set on the neutrino masses.

The three forces of the Standard Model are mediated by vector (spin 1) bosons: the photon $(\gamma)$ for the electromagnetic force, the $W$ and $Z$ bosons for the weak force, and the gluons for the strong force. Attempts to incorporate gravity into the Standard Model have not been successful.

The Standard Model is based on the gauge symmetry group $\mathrm{SU}(3) \times \mathrm{SU}(2) \times \mathrm{U}(1)$, where the $\mathrm{SU}(3)$ group corresponds to $\mathrm{QCD}$ and $\mathrm{SU}(2) \times \mathrm{U}(1)$ corresponds to the electroweak theory. The $W$ and $Z$ bosons acquire mass through the spontaneous symmetry 
breaking of the $\mathrm{SU}(2) \times \mathrm{U}(1)$ symmetry group. To preserve gauge invariance of the theory a scalar particle (the Higgs boson) is introduced [8]. The Higgs couples to both gauge bosons and fermions, and particle masses are governed by the strength of their coupling to the Higgs field. The electroweak coupling $(g)$ of $W$ bosons to fermions is related to the electromagnetic coupling $(e)$ by the following,

$$
g=\frac{e}{\sin \theta_{W}}
$$

where $\theta_{W}$ is the weak mixing angle and $\sin ^{2} \theta_{W}=0.22280 \pm 0.00035[7]$.

The weak-mixing angle also relates the $W$ and $Z$ masses at leading order in the Standard Model by the following,

$$
\sin ^{2} \theta_{W}=1-\frac{M_{W}^{2}}{M_{Z}^{2}}
$$

Furthermore, at the leading order, the theory relates the Fermi constant $\left(G_{F}\right)$ to $g$ by the following,

$$
\begin{aligned}
G_{F} & =\frac{\sqrt{2} g^{2}}{8 M_{W}^{2}} \\
& =\frac{\pi \alpha}{\sqrt{2} M_{W}^{2} \sin ^{2} \theta_{W}}
\end{aligned}
$$

where $\alpha$ is the fine structure constant.

At higher orders there are loop corrections to the $W$ propagator (see Figure 2.1) that change the above equation. Including these and solving for $M_{W}$ gives

$$
M_{W}=\left(\frac{\pi \alpha}{\sqrt{2} G_{F}}\right)^{1 / 2} \frac{1}{\sin \theta_{W} \sqrt{1-\Delta r}}
$$


where $\Delta r$ parametrizes radiative corrections at all orders and has the experimentally determined value of $0.0343 \pm 0.0017$ [7]. The size of the radiative corrections depend in part on the difference in the squared quark masses in the $q \bar{q}$ loop shown in Figure 2.1, thus due to the large difference between the top and bottom quark masses, the $t \bar{b}$ loop dominates. The contribution from the Higgs loop correction goes like $\log M_{\text {Higgs }}$. We can see how these corrections relate to the $W$ mass with the following equation,

$$
\begin{aligned}
M_{W}= & 80.38-0.06 \log \frac{M_{\text {Higgs }}}{100 \mathrm{GeV}}-0.52\left[\frac{\Delta \alpha_{\text {had }}^{5}}{0.03}-1\right] \\
& +0.54\left[\left(\frac{M_{\text {top }}}{175 \mathrm{GeV}}\right)^{2}-1\right]-0.09\left[\frac{\alpha_{s}\left(M_{Z}\right)}{0.12}-1\right]
\end{aligned}
$$

where $M_{\text {top }}$ and $M_{\text {Higgs }}$ are in $\mathrm{GeV}, \Delta \alpha_{\text {had }}^{5}$ is the contribution to $\alpha$ from the five quarks lighter than the top quark, and $\alpha_{s}\left(M_{Z}\right)$ is the strong coupling constant at the $Z$ mass energy scale [9].

From equation 2.6 we can see that for a fixed top mass, higher values of the $W$ mass require smaller values of the Higgs mass, and vice-versa. The nature of this relationship can be more clearly seen in Figure 2.2. Since $M_{\text {top }}$ and $M_{\text {Higgs }}$ enter into the calculation of radiative corrections to $M_{W}$, we can constrain the allowed mass of the Standard Model Higgs by measuring $M_{t o p}$ and $M_{W}$.
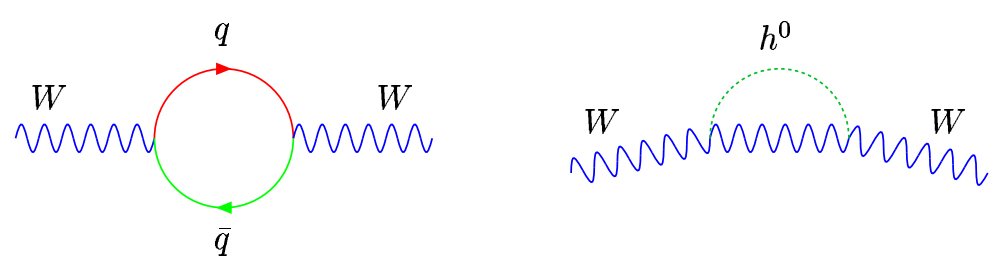

Figure 2.1: First order radiative corrections to the $W$ propagator.

We can see the $M_{W}$ and $M_{t o p}$ measurements favour a lower mass Higgs. Using the 


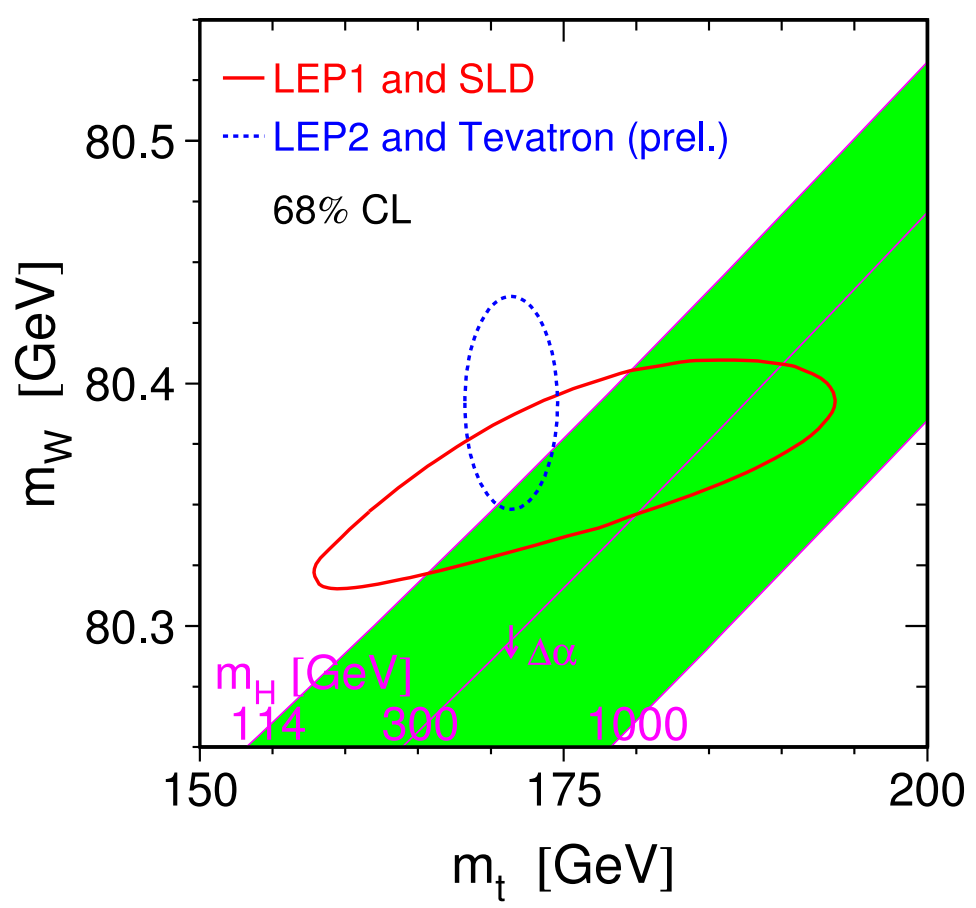

Figure 2.2: Higgs mass constraint from $M_{W}$ and $M_{t o p}$ [3]. A low mass Higgs is favoured.

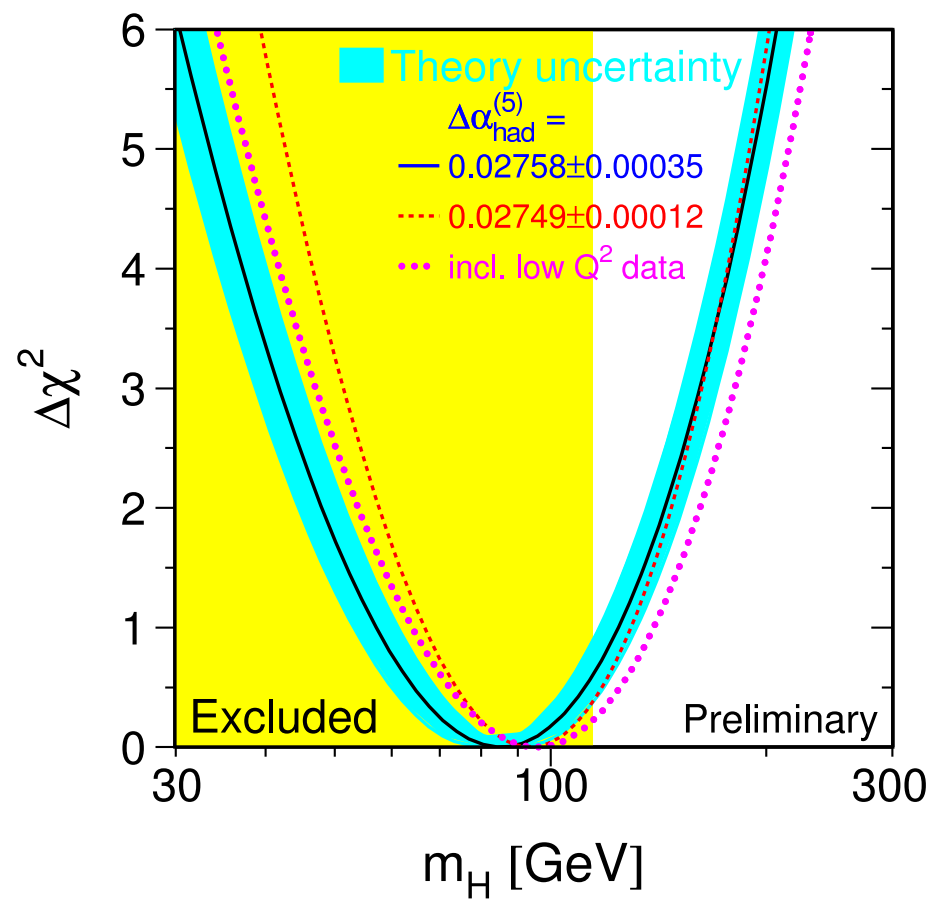

Figure 2.3: Expected Higgs mass from $\chi^{2}$ minimization of Standard Model fits [3]. The shaded region $\left(<114.4 \mathrm{GeV} / c^{2}\right)$ has been excluded by direct searches at LEP. 
new world average top mass value of $(171.4 \pm 2.1) \mathrm{GeV} / c^{2}$ [3], which includes recent CDF and $\mathrm{D} \phi$ measurements, and the world average $W$ mass value shown in Figure 1.1, the Standard Model prediction for the Higgs mass is $89_{-30}^{+42} \mathrm{GeV} / c^{2}$ [3], see Figure 2.3.

\section{1 $W$ Boson Production at the Tevatron}

$W$ bosons are produced at the Tevatron through $p \bar{p}$ collisions at a center-of-mass energy of $1.96 \mathrm{TeV}$, see Figure 2.4.

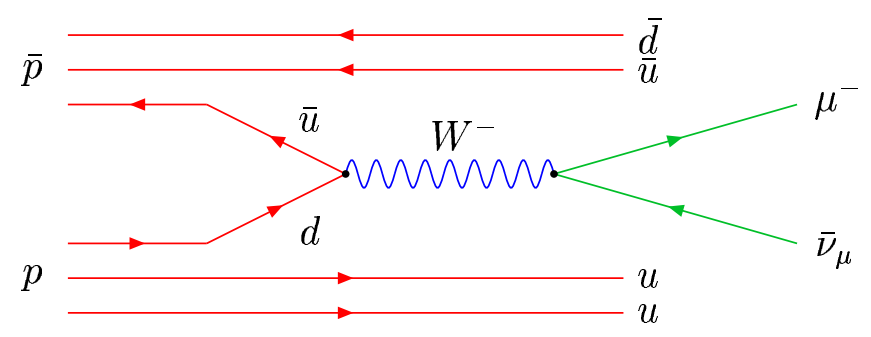

Figure 2.4: Leading order production and decay of a $W$ boson at the Tevatron.

In $p \bar{p}$ collisions at the Tevatron, $W$ bosons are produced via the following modes:

$$
\begin{aligned}
& u \bar{d} \rightarrow W^{+} \\
& \bar{u} d \rightarrow W^{-}
\end{aligned}
$$

These processes involve valence quarks from the proton and antiproton approximately $80 \%$ of the time in Leading-Order (LO) QCD . The other $\sim 20 \%$ of the time the $W$ is produced from two sea quarks [10]. In addition, there are higher order diagrams that contribute to $W$ boson production that are responsible for giving the $W$ boson a non-zero $p_{T}$ spectrum, see Figure 2.5.

The full differential $W$ boson production cross-section at a hadron collider is given by 

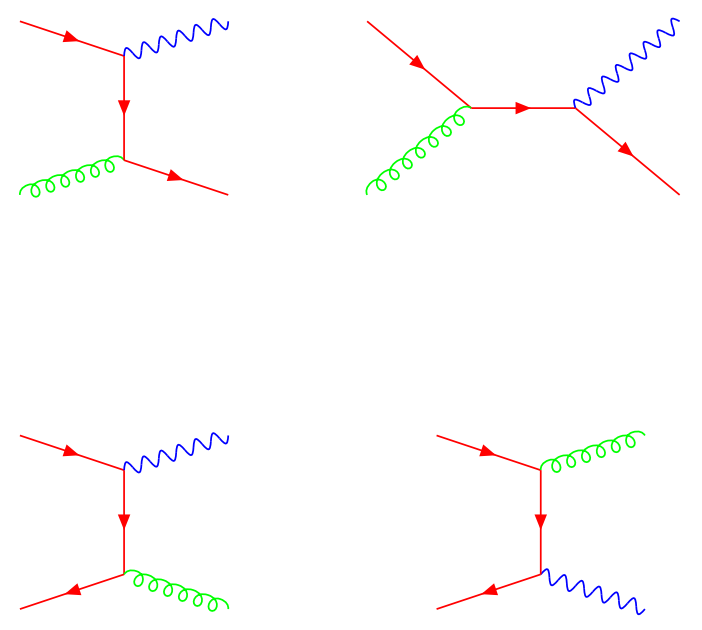

Figure 2.5: Higher order processes contributing to $W$ boson production. Diagrams show quark-gluon fusion (top left), gluon ISR (top right), and two forms of associated gluon- $W$ production (bottom).

$$
\begin{aligned}
\frac{d \sigma}{d q_{T}^{2} d y d \cos \theta d \phi} & =\frac{3}{16 \pi} \frac{d \sigma^{\mu}}{d q_{T}^{2} d y}\left[\left(1+\cos ^{2} \theta\right)\right. \\
& +\frac{1}{2} A_{0}\left(1-3 \cos ^{2} \theta\right)+A_{1} \sin 2 \theta \cos \phi \\
& +\frac{1}{2} A_{2} \sin ^{2} \theta \cos 2 \phi+A_{3} \sin \theta \cos \phi \\
& +A_{4} \cos \theta+A_{5} \sin ^{2} \theta \sin 2 \phi \\
& \left.+A_{6} \sin 2 \theta \sin \phi+A_{7} \sin \theta \sin \phi\right]
\end{aligned}
$$

where the coefficients $A_{0}-A_{7}$ are the helicity amplitudes [11] and $\theta$ and $\phi$ are the polar and azimuthal angles of the charged lepton, respectively, measured in the Collins-Soper frame [12]. This frame is a special $W$ boson rest-frame constructed to normalize the impact of $p_{T}^{W}$ on the angular quantities. Note that the above equation describes $W^{-}$ production; for $W^{+}$one needs to flip the signs of the coefficients $A_{1}, A_{4}$, and $A_{6}$. The $A$ parameters are $p_{T}$ dependent and are influenced by the polarization of the boson. If the $W$ boson is produced with no transverse momentum, the only non-zero parameter is $A_{4}$. Furthermore, if the $W$ is produced only from valence quarks in the proton and 
antiproton, $A_{4}$ equals 2 . If sea-quarks are involved $A_{4}$ becomes less than 2.

In general, the $W$ bosons are produced with a non-zero longitudinal momentum, arising from the different momenta of the constituent quarks and anti-quarks in the proton and antiproton. These momentum distributions are described by the parton distribution functions (PDFs). However, we are not able to measure the longitudinal momentum of the $W$ boson since the hadronic particles produced along with it are lost down the beampipe. This means we do not have sufficient information to reconstruct the invariant mass of the $W$ candidate. We can however measure the energy of the hadronic particles in the direction transverse to the beam direction, which allows us to infer the transverse momentum of the undetected neutrino. Therefore we only use the measurement of kinematic quantities perpendicular to the beam, such as transverse momentum and transverse energy, to determine the $W$ mass. In doing this, the kinematic quantities we use to extract the $W$ mass acquire a unique feature, known as a "Jacobian edge". We can gain some insight into how the Jacobian edge arises by first considering the angular distribution of the charged lepton $\left(l^{\mp}\right)$ in the decay of an at-rest $W$ boson,

$$
\frac{d \sigma}{d \cos \hat{\theta}}=\sigma_{0}(1 \pm \cos \hat{\theta})^{2}
$$

where $\hat{\theta}$ is the angle between the $l^{-}\left(l^{+}\right)$and the incoming anti-quark (quark). Particles and anti-particles have opposite angular distributions; in the equation above the positive sign goes with the $l^{-}$and the negative sign goes with the $l^{+}$.

If we average over positive and negative charges, we get,

$$
\begin{aligned}
\frac{d \sigma}{d \cos \hat{\theta}} & =\sigma_{0}\left(\frac{1}{2}(1+\cos \hat{\theta})^{2}+\frac{1}{2}(1-\cos \hat{\theta})^{2}\right) \\
& =\sigma_{0}\left(1+\cos ^{2} \hat{\theta}\right)
\end{aligned}
$$


If we neglect the masses of the decay products, the magnitude of the momentum of the charged lepton is $p=M_{W} / 2$, since the $W$ boson is at-rest, and the transverse momentum is $p_{T}=\left(M_{W} / 2\right) \sin \hat{\theta}$. Armed with this, let's evaluate the differential cross-section with respect to the $p_{T}$ of the charged lepton,

$$
\begin{aligned}
\frac{d \sigma}{d p_{T}} & =\frac{d \sigma}{d\left(\left(M_{W} / 2\right) \sin \hat{\theta}\right)} \\
& =\frac{2}{M_{W}} \frac{d \sigma}{d \cos \hat{\theta}}\left|\frac{d \cos \hat{\theta}}{d \sin \hat{\theta}}\right| \\
& =\frac{2}{M_{W}} \sigma_{0}\left(1+\cos ^{2} \hat{\theta}\right)\left|\frac{d \cos \hat{\theta}}{d \sin \hat{\theta}}\right|
\end{aligned}
$$

where the factor $\left|\frac{d \cos \hat{\theta}}{d \sin \hat{\theta}}\right|$ is the Jacobian transform and upon evaluation gives $\left(\frac{\sin \hat{\theta}}{\cos \hat{\theta}}\right)$. Using this and eliminating $\sin \hat{\theta}$ and $\cos \hat{\theta}$ with $\sin \hat{\theta}=2 p_{T} / M_{W}$ we get,

$$
\begin{aligned}
\frac{d \sigma}{d p_{T}} & =\frac{2}{M_{W}} \sigma_{0}\left(2-4 p_{T}^{2} / M_{W}^{2}\right)\left(\frac{2 p_{T} / M_{W}}{\sqrt{1-4 p_{T}^{2} / M_{W}^{2}}}\right) \\
& =\frac{4 p_{T}}{M_{W}^{2}} \sigma_{0}\left(2-4 p_{T}^{2} / M_{W}^{2}\right)\left(\frac{1}{\sqrt{1-4 p_{T}^{2} / M_{W}^{2}}}\right)
\end{aligned}
$$

We can see that $\frac{d \sigma}{d p_{T}}$ tends to $\infty$ when $p_{T}=M_{W} / 2$. However, since $M_{W}$ is distributed not as a delta function, rather according to a Breit-Wigner distribution, the cross-section does not become infinite. It does however acquire a sharp asymmetric peak at $M_{W} / 2$, and then rapidly falls off beyond this value. The asymmetry comes from the $p_{T}$ having an upper limit of $M_{W} / 2$. This peak and rapidly falling region is referred to as a Jacobian edge, since it arises from the Jacobian transform used in the above equations. The region around the Jacobian edge contains almost all of the mass information we seek to extract. The peak and edge region is statistically the most powerful part of the distribution. In addition to being broadened by the natural width of the $W$, the edge is also smeared out by detector resolution and non-zero $p_{T}^{W}$. The Jacobian edge is a feature of all three 
kinematic distributions we use to extract the $W$ mass, the advantages and disadvantages of which will be discussed in the following section.

The $W$ decay modes that are used for measuring the $W$ mass at the Tevatron are:

$$
\begin{aligned}
& W^{+} \rightarrow l^{+} \nu_{l} \\
& W^{-} \rightarrow l^{-} \bar{\nu}_{l}
\end{aligned}
$$

The decay process $W \rightarrow l \nu_{l}$ occurs approximately $11 \%$ of the time. The only viable measurement channels are for $l=e$ or $l=\mu$; the $\tau$ channel is plagued by a large QCD background or the complication of multiple neutrinos in the event. Note that the dominant $W$ decay mode is $W \rightarrow q \bar{q}$ which occurs about $68 \%$ of the time. However, the number of particles produced is unpredictable and the detector response to hadrons is more complicated than the response to leptons, so this channel is not used in this analysis.

Experimentally, in $W$ events, we observe a high $p_{T}$ charged lepton and large missing transverse energy, $\mathscr{E}_{T}$, due to the unseen neutrino in the decay. The transverse energy of the neutrino is inferred by measuring the total hadronic activity in the event, referred to as the hadronic recoil $(u)$, see Figure 2.6.

\subsection{Measuring $M_{W}$}

The primary method by which the $W$ mass is extracted from the directly measured quantities is through binned $\log$ likelihood fits to $M_{T}^{W}, p_{T}^{\nu}$, and $p_{T}^{l}$, where $M_{T}^{W}$ is given by

$$
M_{T}^{W}=\sqrt{\left(E_{T}^{l}+E_{T}^{\nu}\right)^{2}-\left(p_{T}^{l}+p_{T}^{\nu}\right)^{2}}
$$




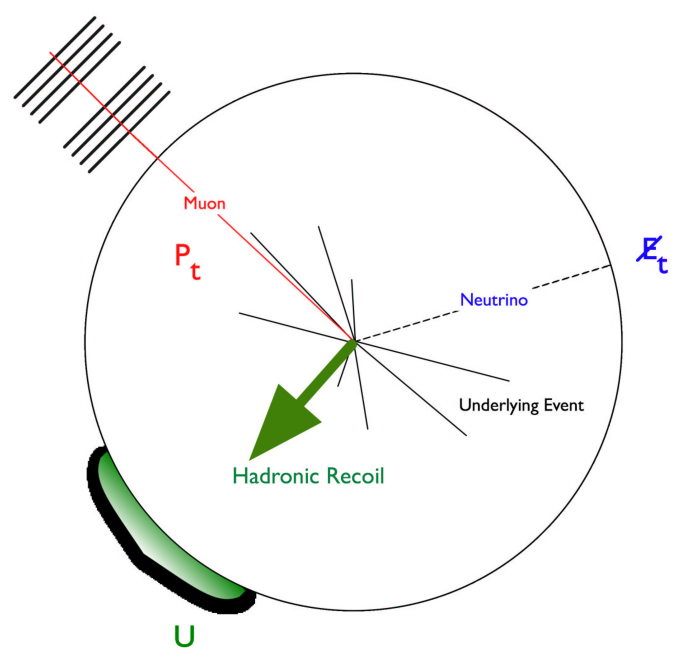

Figure 2.6: Cartoon of $W$ boson decay illustrating the hadronic recoil. The muon appears as hits in the muon chambers. The neutrino escapes undetected.

Traditionally, using $M_{T}^{W}$ has been the preferred method of obtaining the $W$ mass since it is less sensitive to the modelling of the $W p_{T}$ spectrum, which, until recently, had not been calculated with a high degree of accuracy. The best that could be done was to measure the $Z p_{T}$ spectrum and then relate it to a $W p_{T}$ spectrum by means of a theoretical calculation. Even though this method suffered from the combination of limited $Z$ statistics and theoretical uncertainties, it provided better results than a full theoretical calculation. However, larger data samples have greatly improved estimates from calculations that use soft-gluon resummation techniques, especially in the low $p_{T}$ regime. This has made it feasible to use a Monte Carlo generator directly to obtain the $W p_{T} \cdot{ }^{1}$ The $p_{T}^{l}$ technique has benefited the most from these improved calculations as it is sensitive to the $W p_{T}$ at first order.

Even though the transverse mass is not highly sensitive to the $W p_{T}$, it is not without shortcomings. It is quite sensitive to detector related effects such as calorimeter energy

\footnotetext{
${ }^{1}$ Note that in this thesis the term Monte Carlo (MC) generator refers to a computational program that models any one of the production, decay, or interaction of particles. We also use the term to describe the output of one of these programs, e.g. "the data were compared to Monte Carlo templates".
} 
scale and resolution as well as the modelling of the hadronic recoil, since this is used to infer the neutrino $p_{T}$, which comes into the calculation. The main benefit of the $p_{T}^{l}$ technique is that it is not highly sensitive to these experimental effects. These sensitivities are illustrated in Figure 2.7, which shows the muon $p_{T}$ and $W M_{T}$ lineshapes. The black histogram was generated assuming perfect detector resolution and no $W p_{T}$, the blue points are the result of including finite detector resolution, and the shaded grey histogram is the result of including a finite $W p_{T}$ spectrum in addition to finite detector resolution. One can see that transverse mass is most sensitive to detector resolution, whereas the transverse momentum spectrum is most sensitive to $p_{T}^{W}$. With improved calculations of the $W p_{T}$ spectrum, the measurement via $p_{T}^{l}$ now competes with the $M_{T}$ in terms of systematic uncertainties. Measurement via the neutrino from the $W$ decay is sensitive to both the $W p_{T}$ spectrum and detector effects so has largest systematic uncertainty associated with it and thus is often only used as a cross-check.

With higher instantaneous luminosities it will be difficult to model the hadronic recoil due to increased hadronic activity in the event and more multiple interactions per beam-crossing (pile-up). This will degrade the quality of the measurement of $\mathbb{E}_{T}$, which in turn will degrade the measurement of the $W$ mass via $M_{T}^{W}$. Therefore as luminosities increase, the fit to the $p_{T}$ of the charged lepton will become increasingly attractive and may eventually become the primary measurement technique for the $W$ mass [13]. In light of this, it is important to understand now the sources of systematic uncertainty influencing this measurement technique and work towards improving them. An important contribution to to the systematic uncertainty comes from the Monte Carlo modelling of $W$ production and decay. This source of uncertainty is particularly important since it generally depends on improved $\mathrm{MC}$ techniques and generators and therefore will not necessarily decrease with increased statistics. Note that many of the systematic uncertainties on the $W$ mass are determined using other data samples such as $\Upsilon$ mesons, $J / \psi$ 

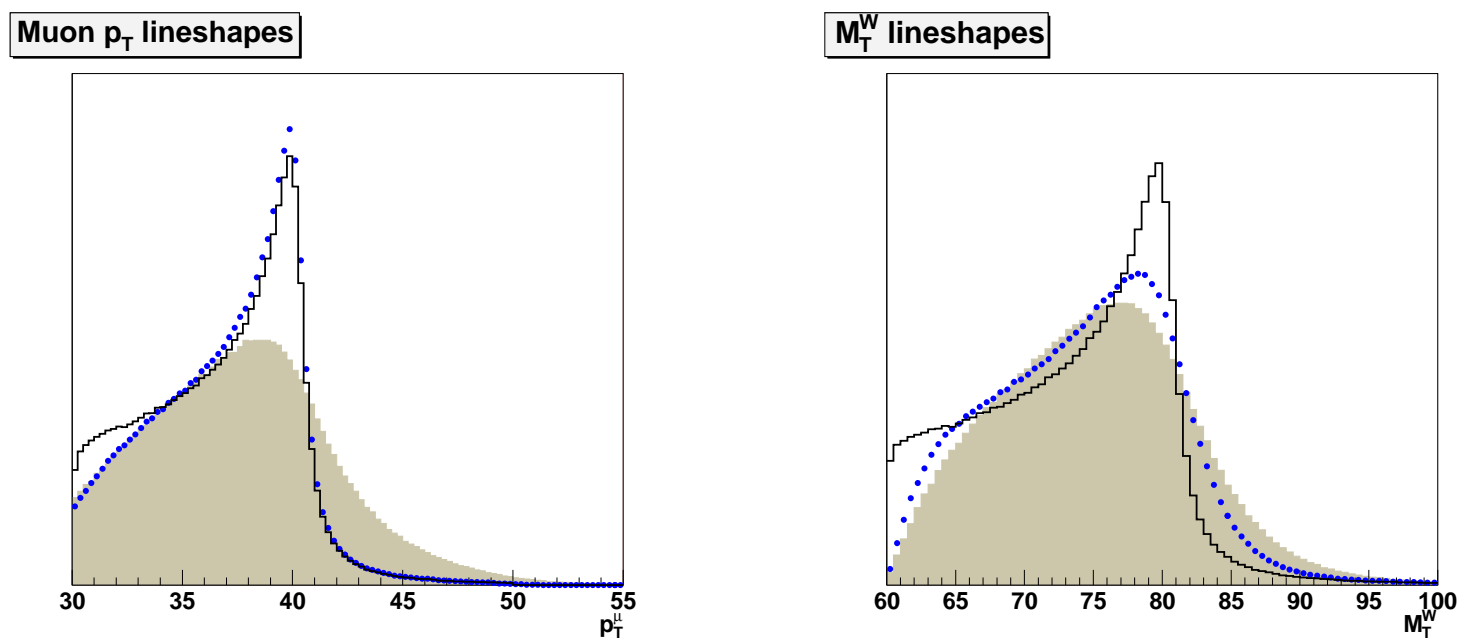

Figure 2.7: Sensitivities of the transverse momentum and transverse mass fits for the $W$ mass. The left plot shows the muon $p_{T}$ spectrum and the right plot shows the transverse mass spectrum of the $W$ boson. The black histogram has perfect detector resolution and no $W p_{T}$, the blue points have finite detector resolution but no $W p_{T}$ spectrum, and the shaded histogram has both finite detector resolution and a finite $W p_{T}$ spectrum.

mesons, and $Z$ bosons. Therefore when the size of these datasets increase, the associated systematic uncertainties tend to decrease. In fact, extrapolations of the uncertainties on the $W$ mass show the uncertainty from MC modelling of $W$ production and decay as the dominant uncertainty with approximately $2 \mathrm{fb}^{-1}$ of data or more. 


\section{Chapter 3}

\section{The CDF II Detector and the Fermilab Tevatron}

This section describes the main components of the accelerator chain at the Fermilab Tevatron and the CDF II detector [14], both of which have been upgraded since the previous run of the experiment over the period 1992-1996, referred to as Run I. The current run of the experiment with both upgraded accelerator facilities and an upgraded detector started in 2001 and is referred to as Run II.

\subsection{The Tevatron Accelerator Chain}

The Fermilab Tevatron is currently the highest energy collider in the world, with $p \bar{p}$ collisions occurring at a center-of-mass energy of $1.96 \mathrm{TeV}$. We describe the production of both protons and antiprotons and their subsequent acceleration and collision.

\subsubsection{Proton Production and Acceleration}

To begin, $H^{-}$ions are obtained by ionizing hydrogen gas. The ions then go to a CockcroftWalton pre-accelerator, which accelerates them to $750 \mathrm{keV}$. The beam of ions is then 
transferred to a linear accelerator (Linac) and further accelerated to $400 \mathrm{MeV}$. See Figure 3.1 for a schematic diagram of the entire Fermilab accelerator complex. The Linac uses a series of $\mathrm{RF}$ cavities and $\mathrm{AC}$ electric fields for acceleration, which also cause the ions to be separated into bunches. As the ions leave the Linac they are passed through a carbon foil to remove the electrons, thus leaving a bunched beam of bare protons. The next phase of proton acceleration takes place in a synchrotron accelerator called the Booster, where the protons are brought up to $8 \mathrm{GeV}$. From there they go to another synchrotron, the Main Injector, where they are further accelerated. Upon reaching $120 \mathrm{GeV}$, some of the protons are aimed at a nickel target for antiproton production, while the rest are further accelerated up to $150 \mathrm{GeV}$.

\subsubsection{Antiproton Production and Acceleration}

As mentioned above, the first stage of antiproton production takes place in the Main Injector, where $120 \mathrm{GeV}$ protons are diverted to a nickel target. This collision produces approximately 1 antiproton for every $10^{5}$ protons on target. The antiprotons are filtered and focused leaving an $8 \mathrm{GeV}$ beam that is sent to the Debuncher and Accumulator rings for stochastic cooling. Stochastic cooling is a procedure that reduces the random momentum spread of the antiproton beam. A pickup electrode is used to detect a particle's deviation from the ideal orbit and then sends a signal to a "kicker magnet" that bends the particle back towards the desired orbit. Once they are cooled, the antiprotons are bunched and sent back to the Main Injector and accelerated up to $150 \mathrm{GeV}$.

\subsubsection{The Tevatron}

From the Main Injector, 36 proton and 36 antiproton bunches are injected into the Tevatron where they are accelerated to their final energies of $980 \mathrm{GeV}$. The Tevatron is a superconducting synchrotron approximately $1 \mathrm{~km}$ in radius. The proton and antiproton 


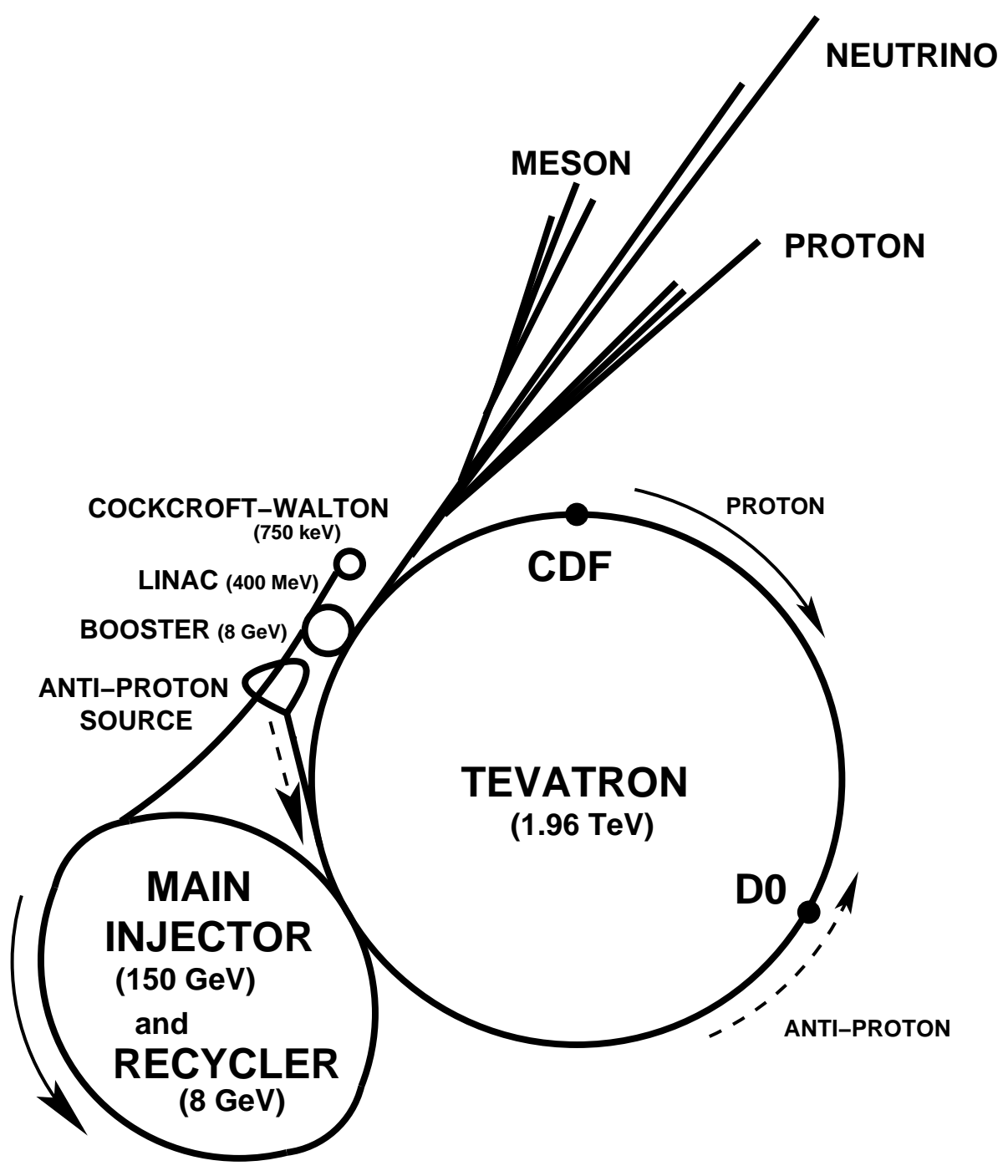

Figure 3.1: Fermilab accelerator complex.

beams are both inside the Tevatron, but move in opposite directions. With the aid of superconducting magnets the beams are brought to collision at two points on the ring: one at the CDF II detector and one at the $D \phi$ detector. The collision energy is $1.96 \mathrm{TeV}$.

The number of events $N$ produced for a physics process with cross-section $\sigma$ over some time $\Delta t$ is given by,

$$
\begin{aligned}
N & =\sigma \int_{\Delta t} L d t \\
& =\sigma \mathcal{L}
\end{aligned}
$$


where $L$ is the instantaneous luminosity and $\mathcal{L}$ is the integrated luminosity. As of the writing of this thesis the the Tevatron routinely achieves instantaneous luminosities over $1.5 \times 10^{32} \mathrm{~cm}^{-2} \mathrm{~s}^{-1}$ and has delivered approximately approximately $1600 \mathrm{pb}^{-1}$ of data to the CDF II detector since the beginning of Run II.

\subsection{The CDF II Detector}

The CDF II detector is a general-purpose solenoidal detector which has been upgraded to accommodate the higher luminosities and beam conditions in Run II, see Figure 3.2. Data taking for Run II began in March 2001 and and as of the writing of this thesis the CDF II detector has recorded approximately $1300 \mathrm{pb}^{-1}$ of data. Note that the difference between delivered luminosity and recorded luminosity arises from the approximate $80 \%$ data taking efficiency of the CDF II detector.

As can be seen in the schematic view shown in Figure 3.2, the detector is cylindrically symmetric about the $p \bar{p}$ beamline. In general, the detector consists of tracking chambers closest to the beamline for precise tracking of charged particles, followed by calorimeter modules at larger radii for the measurement of particle energies, and finally beyond that a series of drift chambers used to detect muons. The tracking chambers are immersed in a uniform magnetic field of $1.4 \mathrm{~T}$ provided by a $5 \mathrm{~m}$ long superconducting solenoid located outside the tracking region.

\subsubsection{The CDF Coordinate System and Transverse Quantities}

A cylindrical coordinate system is used at $\operatorname{CDF}(\theta, \phi, z)$ with the origin at the $p \bar{p}$ interaction point. The $z$ axis is defined to be along the incoming proton direction and the polar angle $\theta$ is measured with respect to that. The azimuthal angle $\phi$ is defined in the plane perpendicular to the $z$ axis, the $x y$ plane, where $x$ is oriented radially outward 


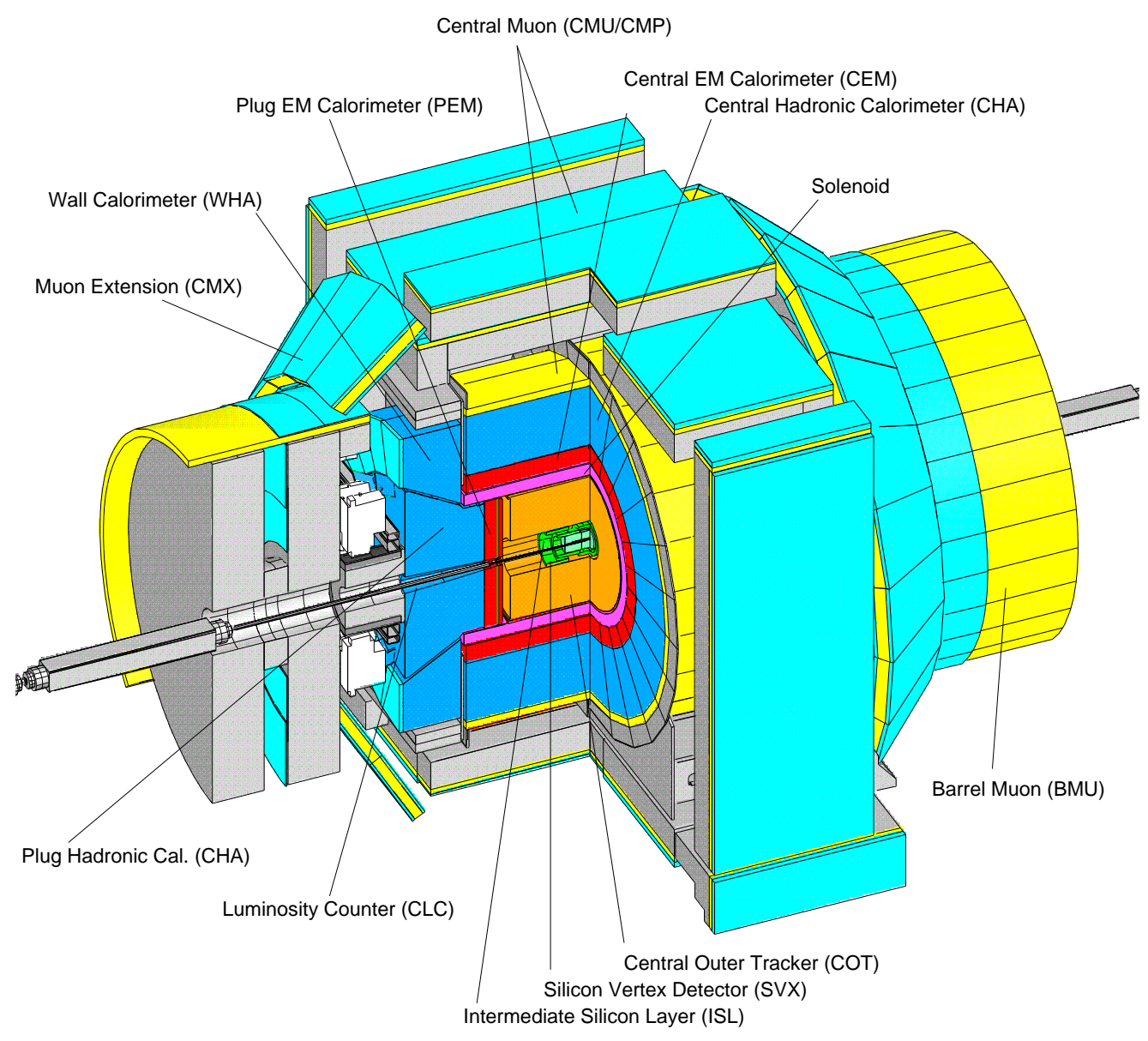

Figure 3.2: CDF II detector.

from the center of the Tevatron and $y$ vertically upwards. It is also useful to define a quantity called the pseudorapidity given by $\eta=-\ln \tan (\theta / 2)$ since it transforms linearly under boosts in the $z$ direction. For massless particles the pseudorapidity is equal to the rapidity, given by, $y=\frac{1}{2} \ln \frac{E+p_{z}}{E-p_{z}}$. In terms the pseudorapidity, the detector covers the region $\eta=0$ to approximately $|\eta|=3.6$.

As discussed in the previous chapter, we make use of measurements of both momentum and energy in the transverse plane, with $p_{T}=p \sin \theta$ and $E_{T}=E \sin \theta$. In addition, the missing transverse energy is defined as $\mathbb{H}_{T}=-\left|\Sigma_{i} E_{T}^{i} \hat{n}_{i}\right|$, where $\hat{n}_{i}$ are unit vectors in the azimuthal plane pointing from the reconstructed event vertex to the $i^{\text {th }}$ calorimeter tower. The missing transverse energy in the calorimeter is corrected to account for the 
minimum ionizing energy $(\sim 2 \mathrm{GeV})$ deposited by muons as they pass through.

\subsubsection{Charged Particle Tracking Systems}

Accurate charged particle tracking is crucial to the measurement of the $W$ mass. The tracking system at CDF consists of a silicon micro-strip detector nearest to the beamline surrounded by an open-cell wire drift chamber, see Figure 3.3. The silicon detector provides full coverage up to $|\eta|<2$ while the drift chamber covers $|\eta|<1$.

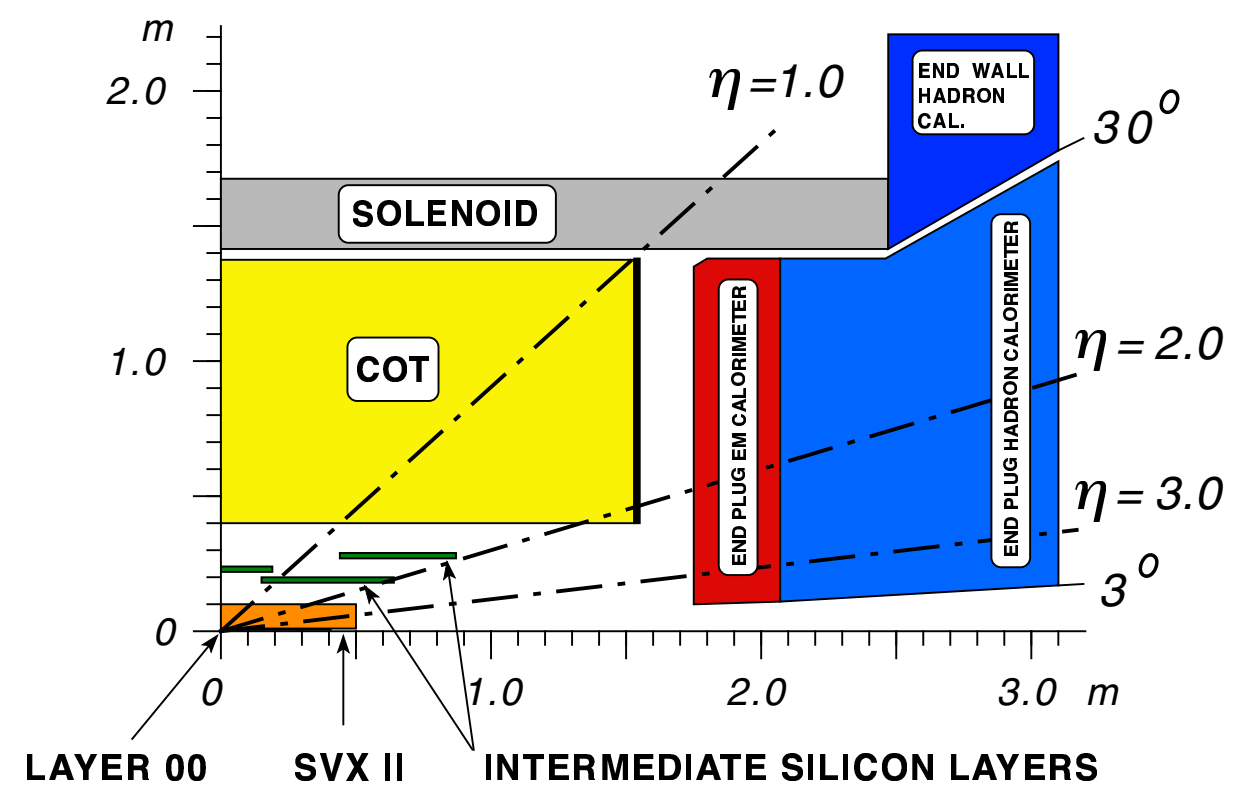

Figure 3.3: Tracking systems at CDF. The silicon detector consists of Layer 00, SVX II, and the Intermediate Silicon Layers. The drift chamber is the COT.

\section{Silicon Tracking Detectors}

The CDF II silicon detector system only affects the $W$ mass analysis through its interaction with particles that pass through it. For example, as particles traverse the silicon they can undergo multiple scattering, ionization energy loss, bremsstrahlung, or photon con- 
versions. We do not actually use it for tracking charged particles. This is accomplished entirely by a large drift chamber, which will be described next.

\section{Drift Chamber}

All of the charged particle tracking in the $W$ mass measurement is provided by the Central Outer Tracker (COT), a $310 \mathrm{~cm}$ long open-cell drift chamber extending between $40 \mathrm{~cm}$ and $132 \mathrm{~cm}$ from the beampipe. The principle behind tracking in the drift chamber is that when a charged particle transverses the chamber, it ionizes a gas, whereupon the ion pairs are directed towards sense wires by an electric field, allowing for detection. An algorithm is used to search for a continuous pattern of localized charge depositions from the ion pairs (hits). From this the charged particle's trajectory (track) can be reconstructed.

The COT consists of 8 "superlayers", each of which has 12 sense wires. Superlayers alternate between axial (sense wires parallel to $z$ axis) and stereo (sense wires strung at angles $\pm 2^{\circ}$ with respect to the $z$ axis), with the first layer being stereo, see Figure 3.4. The axial layers provide solely $r-\phi$ measurements of the particle's track while the stereo layers allow for measurements in $z$ as well. Each superlayer has "cells" that contain potential wires to set up the necessary electric field, sense wires to detect ions created from the particle passing through, and shaper wires used to shape the electric field properly, see Figure 3.5.

The COT is filled with a gas mixture of $50 \%$ Argon and $50 \%$ ethane. This mixture was chosen to ensure fast drift velocities $(\sim 100 \mu \mathrm{m} / \mathrm{ns})$ so that the maximum drift time is less than the $396 \mathrm{~ns}$ beam crossing time. This is achieved since the maximum drift distance is about $0.88 \mathrm{~cm}$ making for maximum drift times of about $177 \mathrm{~ns}$, less than half the bunch crossing time.

The charge and momentum of the reconstructed tracks are measured from the curvature in the COT. The transverse momentum of reconstructed tracks is obtained from 


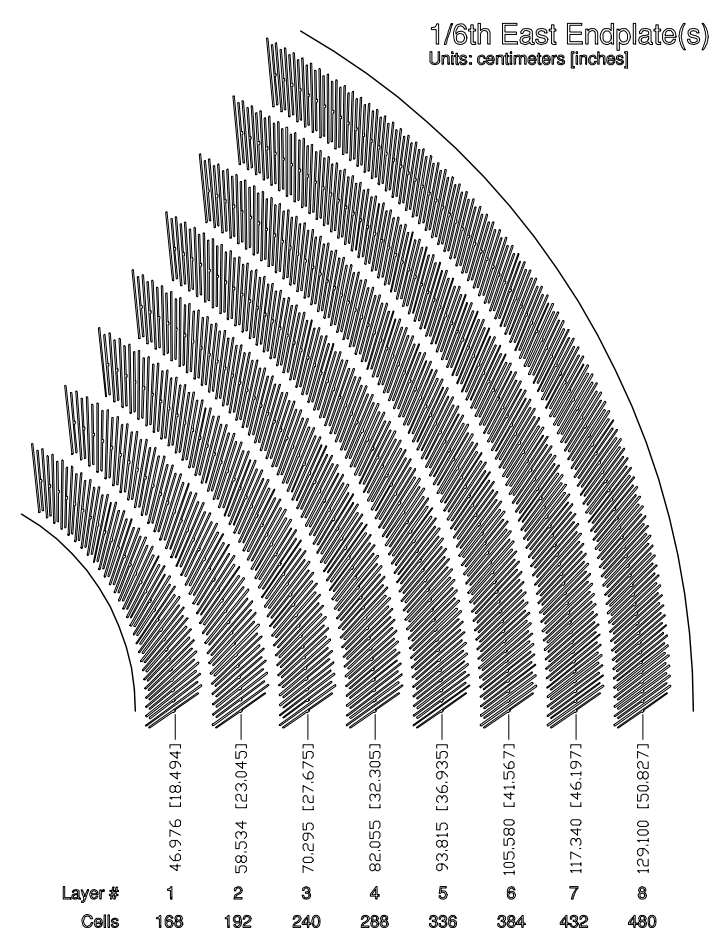

Figure 3.4: Portion of the COT illustrating the arrangement of superlayers. Layers alternate between stereo and axial, starting with layer 1 as stereo.

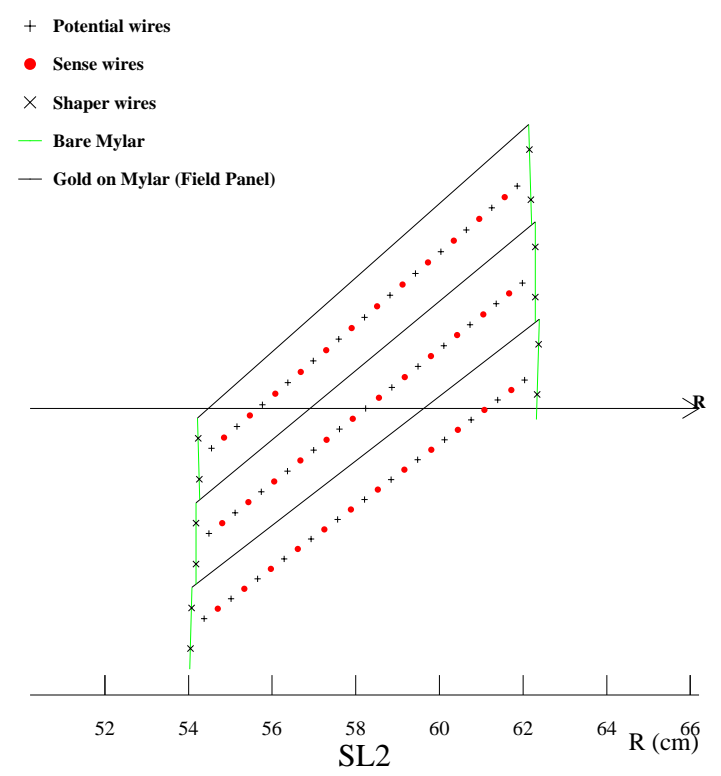

Figure 3.5: View of superlayer 2 along the $z$ direction. Three supercells are shown, illustrating the wire configuration within a cell. 
the equation $p_{T}=0.3 B r$, where $B$ is the magnetic field strength, and $r$ is the radius of curvature. When $B$ is in Tesla and $r$ is in $\mathrm{m}, p_{T}$ has units of $\mathrm{GeV}$.

The COT momentum resolution is studied by measuring the difference in curvature between reconstructed cosmic tracks as they enter the detector volume (inward legs) and as they leave (outward legs). The two legs belong to the same track so the only curvature difference is due to resolution, once the energy loss in the silicon detector has been accounted for. A COT momentum resolution of $\sigma_{p_{T}} / p_{T}^{2} \approx 1.7 \times 10^{-3}(\mathrm{GeV} / c)^{-1}$ is measured. We can see that higher $p_{T}$ tracks have worse momentum resolution, since they bend less in the magnetic field. The amount of material in the COT corresponds to approximately 0.016 radiation lengths $\left(X_{0}\right)$ which is important for calculating energy loss and multiple scattering.

\subsubsection{Calorimeter Systems}

The CDF calorimeter is a sampling scintillator calorimeter designed to measure the energy of both charged and neutral particles produced in $p \bar{p}$ collisions. The calorimeter modules are located outside the tracking system and consist of alternating layers of active material (scintillator) sandwiched between layers of passive (absorbing) material such as lead or steel. Particles deposit their energy in the calorimeter through "showers" of secondary particles produced when the primary particle interacts with the material in the calorimeter. Light guides are used to collect photons produced from charged particles passing through the scintillator material, which are then directed to photomultiplier tubes.

The CDF calorimeter system is divided into two regions: the central region $(|\eta|<1)$, which consists of the Central Electromagnetic calorimeter (CEM) and outside that the Central Hadronic calorimeter (CHA), and the plug region $(1.1<|\eta|<3.6)$, consisting of the Plug Electromagnetic calorimeter (PEM) and outside that the Plug Hadronic calorimeter (PHA). The reason that the electromagnetic sections of the calorimeter come 
before the hadronic sections is that electromagnetic showers begin after traversing less material than hadronic showers. Hadrons will typically traverse the entire EM section of the calorimeter before depositing their energy in the hadronic section. The total energy deposited in the entire calorimeter (EM and hadronic sections) is obtained by summing the energies in each calorimeter tower. There are 480 calorimeter towers in the central region as well as 480 towers in the plug region.

\section{Central Calorimeter System (CEM, CHA, WHA)}

The CEM consists of azimuthal wedges covering $0.1 \times 15^{\circ}$ in $\eta \times \phi$ space. Wedges consist of 31 layers of $0.125 \mathrm{~cm}$ of lead interleaved with $0.5 \mathrm{~cm}$ of polystyrene scintillator. Each wedge is grouped into 10 readout towers (calorimeter towers) arranged in a projective geometry, see Figure 3.6. The CEM is approximately $18 X_{0}$ deep.

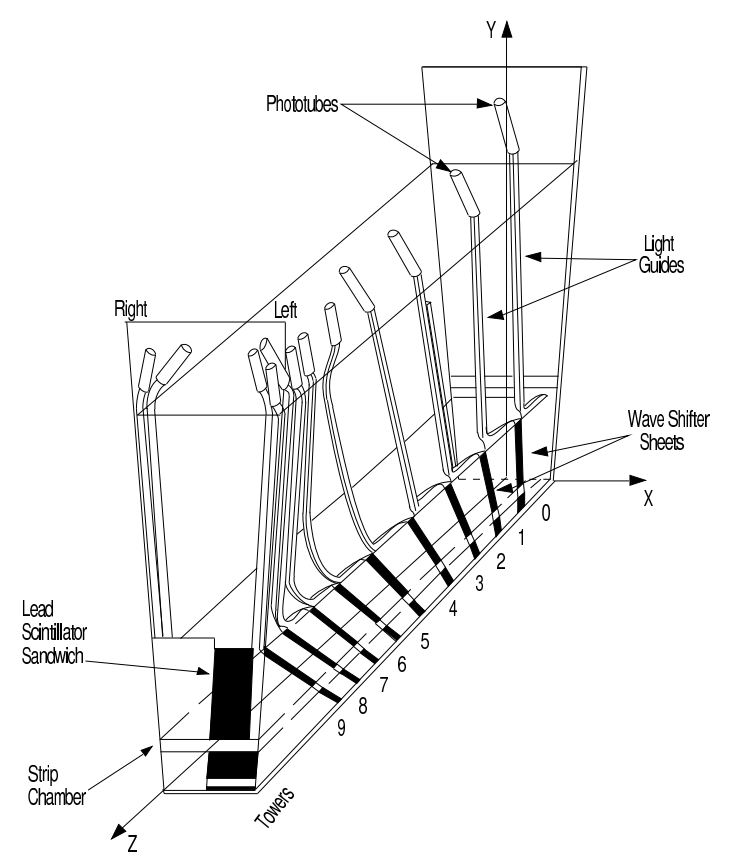

Figure 3.6: A wedge of the central calorimeter.

The CHA is directly outside of the CEM and consists of 32 layers of $2.5 \mathrm{~cm}$ iron interleaved with $1.0 \mathrm{~cm}$ of scintillator. The CHA is approximately 4.5 interaction lengths 
$\left(\lambda_{0}\right)$ deep. Finally, between the central calorimeter system and the plug calorimeter system is the Wall Hadronic Calorimeter (WHA) which has 15 layers of $5.0 \mathrm{~cm}$ iron and $1.0 \mathrm{~cm}$ of scintillator and is approximately $4.5 \lambda_{0}$ deep.

\section{Plug Calorimeter System (PEM, PHA)}

The construction of the PEM is analogous to the CEM with 23 alternating layers of lead $(0.4 \mathrm{~cm})$ and scintillator $(0.4 \mathrm{~cm})$. The segmentation in $\phi$ is $15^{\circ}$ (same as CEM) but is doubled to $7.5^{\circ}$ in the region $1.1<|\eta|<2.1$. The PEM is approximately $21 X_{0}$ deep (at normal incidence). The PHA is located directly outside the PEM and consists of 23 alternating layers of iron $(2.5 \mathrm{~cm})$ and scintillator $(1.0 \mathrm{~cm})$. The PHA is approximately $7 \lambda_{0}$ deep.

\subsubsection{Muon Chambers}

Since muons only lose energy through electromagnetic interactions and are much more massive than electrons, they can travel through significantly more material than other charged particles. For this reason the muon detectors are the outermost particle detectors at CDF. Muons passing through the detector leave a track in the COT and deposit a small fraction of their energy in the calorimeter (about $0.4 \mathrm{GeV}$ in the EM section and about $2 \mathrm{GeV}$ in the hadronic section on average).

The muon system at CDF consists of several subdetectors: the Central Muon Chambers (CMU) which cover $0<|\eta|<0.6$, directly outside that in radius, the Central Muon Upgrade (CMP), covering the same $\eta$ range, and finally the Central Muon Extension (CMX) which covers $0.6<|\eta|<1.0$, see Figure 3.2. In order not to be absorbed, muons must have a minimum $p_{T}$ of $1.4 \mathrm{GeV} / c$ to reach the $\mathrm{CMU}$; to reach the CMP they must have at least $2.2 \mathrm{GeV} / c$, otherwise they will be absorbed in either the calorimeter system or additional steel absorber between the CMU and CMP.

A new subdetector, the Barrel Muon Upgrade (BMU), covering $1.0<|\eta|<1.5$ was 
being commissioned during the data taking period of this analysis and therefore was not used.

The CMU subdetector is segmented into 24 east and 24 west $15^{\circ}$ wedges, see Figure 3.7. Each wedge contains three muon chambers (parallel to the $\phi$ direction). The wedges consist of four layers of drift cells in the radial direction, see Figure 3.8.

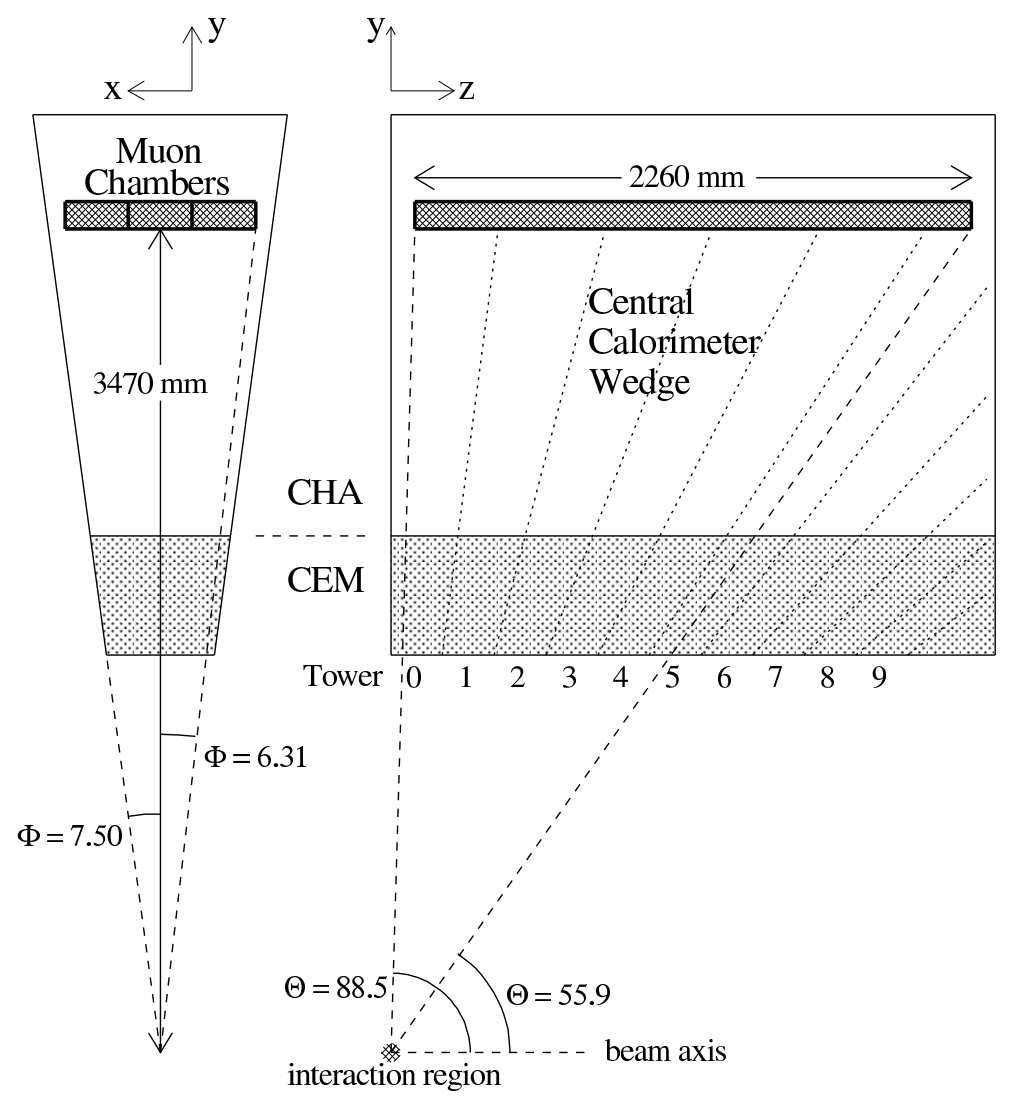

Figure 3.7: Schematic drawing of the muon detectors located behind the central calorimeter.

Each cell has a $50 \mu \mathrm{m}$ sense wire strung through the cell center, parallel to the $z$ axis. The cells are filled with a mixture of Argon and ethane gas and the hits for a track in the muon chambers (called a "stub") are recorded by measuring the drift times of ions to the sense wire. In the CMU the maximum drift time is $800 \mathrm{~ns}$, which is longer than the 


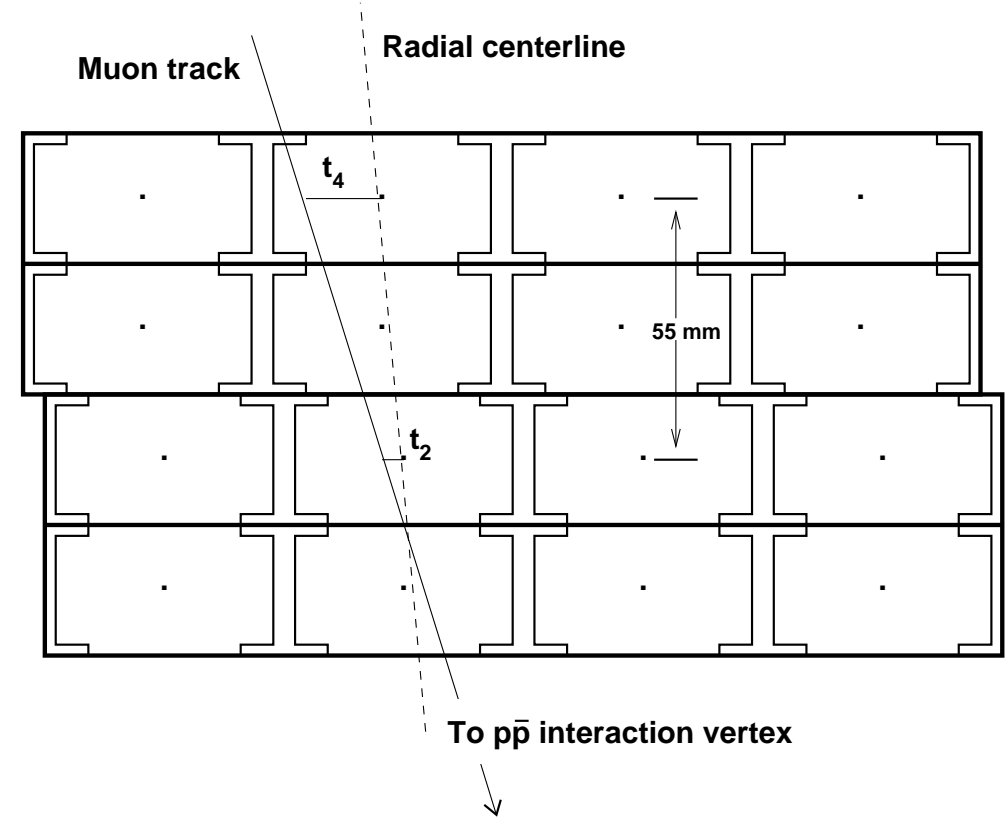

Figure 3.8: Muon chamber in the CMU.

396 ns bunch crossing time, thus trigger and offline reconstruction information are used to uniquely determine from which bunch crossing the hit originated. Muon candidates are formed by matching a stub to an extrapolated COT track.

The CMP subdetector is arranged in a box-like configuration around the CMU with an additional $3 \lambda$ of steel absorber sandwiched between the two, see Figure 3.2. The additional material greatly reduces the background coming from hadrons misidentified as muons in the muon chambers. The CMP is often used in conjunction which the CMU to improve purity of muon samples (the combination of the two is often referred to as the CMUP). Located just outside of the CMP is a scintillator, called the Central Scintillator Upgrade (CSP).

The CMX subdetector provides muon coverage in the plug region and is similar in construction to the CMU. However, the CMX has eight concentric layers, compared to only four in the CMU. The cells are slightly staggered with respect to each other to provide measurements in $z$ from the different stereo angles of each cell with respect to 
the beam axis. Analogous to the CMP, a scintillator is located outside of the CMX, called the CSX.

\subsubsection{Cherenkov Luminosity Counter}

At CDF, the instantaneous luminosity is measured using Cherenkov Luminosity Counters, one located at either end of the detector near the plug calorimeter system. They are located close to the beamline and consist of 48 Cherenkov counters filled with isobutane. They measure the number of inelastic $p \bar{p}$ collisions by measuring the number of particles emerging from the collision at small angles. From this the luminosity is calculated.

\subsubsection{Data Acquisition System}

The CDF Data Acquisition System consists of a three level trigger system that selects events we are interested in and writes them to disk for further analysis offline. The trigger system reduces the rate at which data are recorded from the $2.5 \mathrm{MHz}$ beam crossing rate to $75 \mathrm{~Hz}$, the typical rate at which data can be stored on disk. This corresponds to an event reduction rate of about 1:30000. See Figure 3.9 for a schematic diagram of the CDF trigger system.

The first level of the trigger system (L1) is a purely hardware based trigger that uses information from the calorimeters, tracking chambers, and muon detectors to make a decision to accept or reject the event. Collision data are fed into $6 \mu$ s pipelines and the global L1 decision must be made and returned to the front-end electronics before the data reach the end of the pipelines. Information from the COT is processed by the XFT (extremely fast tracker) to aid in the L1 decision. The L1 trigger reduces the event rate from $2.5 \mathrm{MHz}$ to less than $20 \mathrm{kHz}$. Events accepted at L1 are transferred to one of four L2 buffers.

At L2, all the L1 information is available as well as two dedicated pieces of hardware: the cluster finder, which merges energies in neighbouring calorimeter towers to form 
clusters, and the SVT (silicon vertex tracking hardware) which uses silicon hits to search for tracks with displaced vertices. Finally, data are fed into a processor board which uses simple selection algorithms to make a L2 decision. The maximum L2 accept rate is 300 $\mathrm{Hz}$.

The final level of the trigger system, L3, consists of a processor farm of about 300 PCs that run optimized versions of the offline reconstruction code and impose loose cuts on the reconstructed events. The L3 algorithms include track reconstruction, track matching with calorimeter energy clusters, and muon stub reconstruction. In general, the L3 trigger makes use of all detector information to reconstruct events. Events accepted at L3 are written to disk at the rate of $75 \mathrm{~Hz}$. 


\subsection{MHz Crossing rate}

132 ns Bunch Spacing (2.5 MHz / 396 ns)

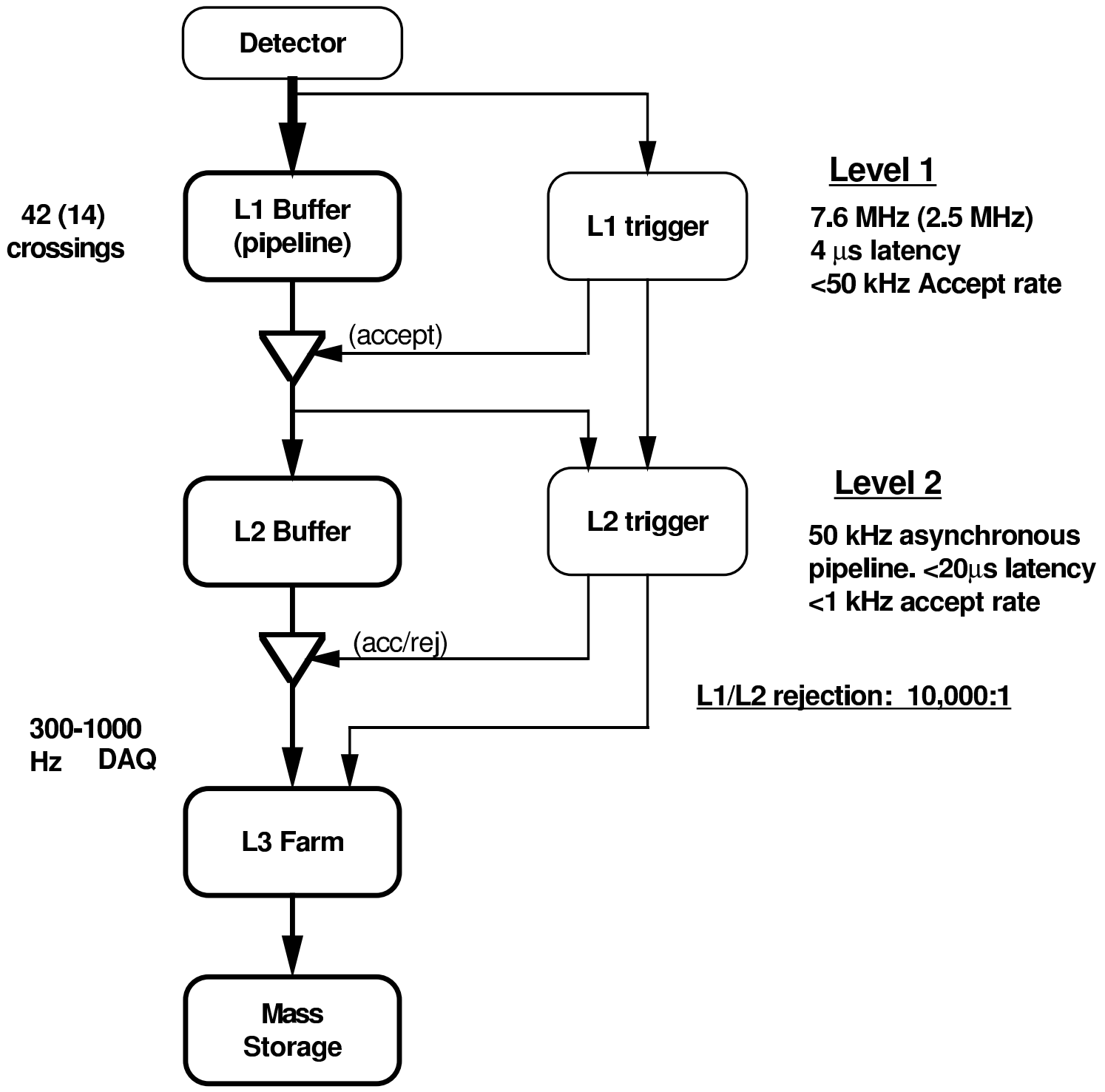

Figure 3.9: CDF trigger system. 


\section{Chapter 4}

\section{Event Generation}

Event generation is an important aspect of the $W$ mass analysis despite the relatively small magnitude of the systematic uncertainties associated with it. The reason is that the uncertainties arising from event generation generally do not scale with increased data statistics, and therefore will eventually come to be one of the dominant uncertainties in future higher statistics measurements of the $W$ mass [13].

In a broad sense, there are two relevant aspects of event generation: QED physics and QCD physics. QED physics encompasses processes such as photon radiation and EWK corrections and QCD physics includes processes such as gluon radiation.

We begin by generating large Monte Carlo samples of $W$ events in order to construct templates that are used to fit the data for the $W$ mass. We use the generator RESBOS [15] to create samples of $p \bar{p} \rightarrow W \rightarrow l \nu$ events. RESBOS includes Next-to-LeadingOrder (NLO) processes such as gluon radiation that give rise to a non-zero $p_{T}^{W}$ spectrum. In addition, it uses a technique called soft-gluon resummation, which aids in modelling the low $p_{T}$ spectrum that is relevant for our measurement of the $W$ mass. We account for QED processes using the program WGRAD [16], which contains $\mathcal{O}(\alpha)$ electroweak corrections to $W \rightarrow l \nu$ including a real final state photon.

In this chapter we discuss the physics associated with both QCD and QED in turn, as 
well as present the systematic uncertainties on the $W$ mass. Parton distribution functions (PDFs) are discussed at the end of this chapter. Note that in this chapter, results for the electron channel are presented on equal footing with the muon channel. The reason being that the procedure for generating events and estimating systematic uncertainties is the same for both. In addition, the electron channel results provide many valuable comparisons to the muon channel. Also for comparison purposes we provide results for all three $W$ mass fit variables $\left(p_{T}, M_{T}^{W}, \mathbb{E}_{T}\right)$.

\subsection{QED Physics}

In past measurements of the $W$ mass [17], QED radiative corrections were accounted for via a Berends and Kleiss [18] calculation. This included one real photon in the final state, and used approximate calculations for the soft and virtual terms. The impact of a second final state photon was treated using the program PHOTOS [19]. These QED radiative effects were not included in the $W$ mass fitting templates, instead a correction was made to the fitted $W$ mass to account for them.

In this analysis, we treat the relevant QED processes more thoroughly by making use of the $\mathcal{O}(\alpha)$ MC generator WGRAD to generate $W \rightarrow l \nu$ and including the effects of QED radiation in the lineshapes of our $W$ mass fitting templates.

This section is organized as follows: we first discuss the physics behind WGRAD and some issues regarding using WGRAD as an event generator. We then investigate shifts in the $W$ mass caused by various QED process, including the changes to the lineshapes of the fit variables. Then a procedure is outlined for combining QED corrections with the templates used to fit the $W$ mass. We briefly mention a strategy for modelling QED for $Z$ events. Finally we summarize all the sources of systematic uncertainty arising from QED. 


\subsubsection{QED with WGRAD}

WGRAD calculates $W$ boson production and decay with $\mathcal{O}(\alpha)$ electroweak corrections. It includes the real photon contribution, which can come from one of three types of radiation: radiation off the incoming quarks (ISR), radiation off the final state charged lepton (FSR), or radiation off the propagator. In general, the calculations in WGRAD are carried out by forming gauge invariant sets of various types of corrections. The $W$ decay can be selected as either to the electron or muon channel. One has the option of running WGRAD in various modes: ISR only, FSR only (using the full treatment or using the Berends and Kleiss approximation), interference only (that is, interference terms between FSR and ISR), full QED, or leading order (LO), i.e. Born level process only. These switches are useful in analyzing the impact of the various types of QED radiation independently. Note that WGRAD does not contain any QCD corrections and thus all events have $p_{T}^{W}=0$.

WGRAD is based on a modified version of VEGAS [20]. There are two stages in the calculation of $W$ events with WGRAD: a first pass in which the integration grid maps to peaks in the cross section, then a second pass in which a precision calculation is carried out and event weights are generated. We use a procedure to unweight the events so we can use WGRAD as an event generator [21]. This involves storing the maximum weight, $w_{\text {max }}$, each time the precision calculation is performed. Then multiple calls are made to the integration routine and the calculated weight, $w$, is compared to $w_{\max }$. If the following condition is satisfied the event is "unweighted", i.e. receives unit weight,

$$
w / w_{\max }>\mathcal{R}[0,1]
$$

where $\mathcal{R}[0,1]$ is a random number between 0 and 1 . Since $w$ is usually quite small the unweighting process is slow. Events that have $w>w_{\max }$ may get stored multiple times. A small fraction of weights generated are negative $(\sim 0.05 \%)$; these events are treated the same as positive weight events, however, if stored receive a weight of -1 [21]. 
In WGRAD, a cut is made on the energy of the photon in order to avoid divergences as $E_{\gamma} \rightarrow 0$. The photon energy cut, controlled by the parameter $\delta_{s}$, is given by

$$
E_{\gamma}>\delta_{s} \sqrt{\hat{s}} / 2
$$

where $E_{\gamma}$ is the energy of the emitted photon in the parton-parton center of mass frame and $\sqrt{\hat{s}}$ is the partonic center-of-mass energy.

Choices of $\delta_{s}$ affect the number of three body final states $(l \nu \gamma)$ generated. For example, in full QED mode, $\delta_{s}=0.01$ for the muon channel gives about $12 \%$ of the final states with a photon; whereas $\delta_{s}=0.001$ gives about $20 \%$ of the final states with a photon. A $\delta_{s}$ of 0.001 corresponds to a minimum photon energy of approximately 150 $\mathrm{MeV}$. We examine the impact of the choice of $\delta_{s}$ on the measured $W$ mass later.

There are numerous features of WGRAD that could potentially be investigated further, however, for our purposes we are concerned with the effect of including QED corrections via WGRAD on the fit variables used to extract the $W$ mass. Figure 4.1 shows the $M_{T}^{W}$ and $p_{T}^{l}$ distributions for electrons and muons at Born level and including $\mathcal{O}(\alpha)$ electroweak corrections via WGRAD. Electroweak corrections reduce the height of the Jacobian peak in both distributions. Since corrections are of order $\ln \left(\sqrt{\hat{s}} / m_{l}^{2}\right)[16]$ the effect is larger for the electron channel. Photon FSR reduces the invariant mass of the $l \nu$ system, thus shifting the location of events from the peak region in both distributions to lower values. Note that these changes are significant in the $M_{T}^{W}\left(60-90 \mathrm{GeV} / c^{2}\right)$ and $p_{T}^{l}(30-45 \mathrm{GeV} / c)$ regions that are used for extracting the $W$ mass.

\subsubsection{Mass Shifts}

In order to gauge how various QED processes impact our measurement of the $W$ mass we use the fast detector simulation described in chapter 6 and the fitter described in chapter 8. However, instead of fitting data to the high statistics templates generated by the fast simulation, we input a Monte Carlo "data" file with equally high statistics 

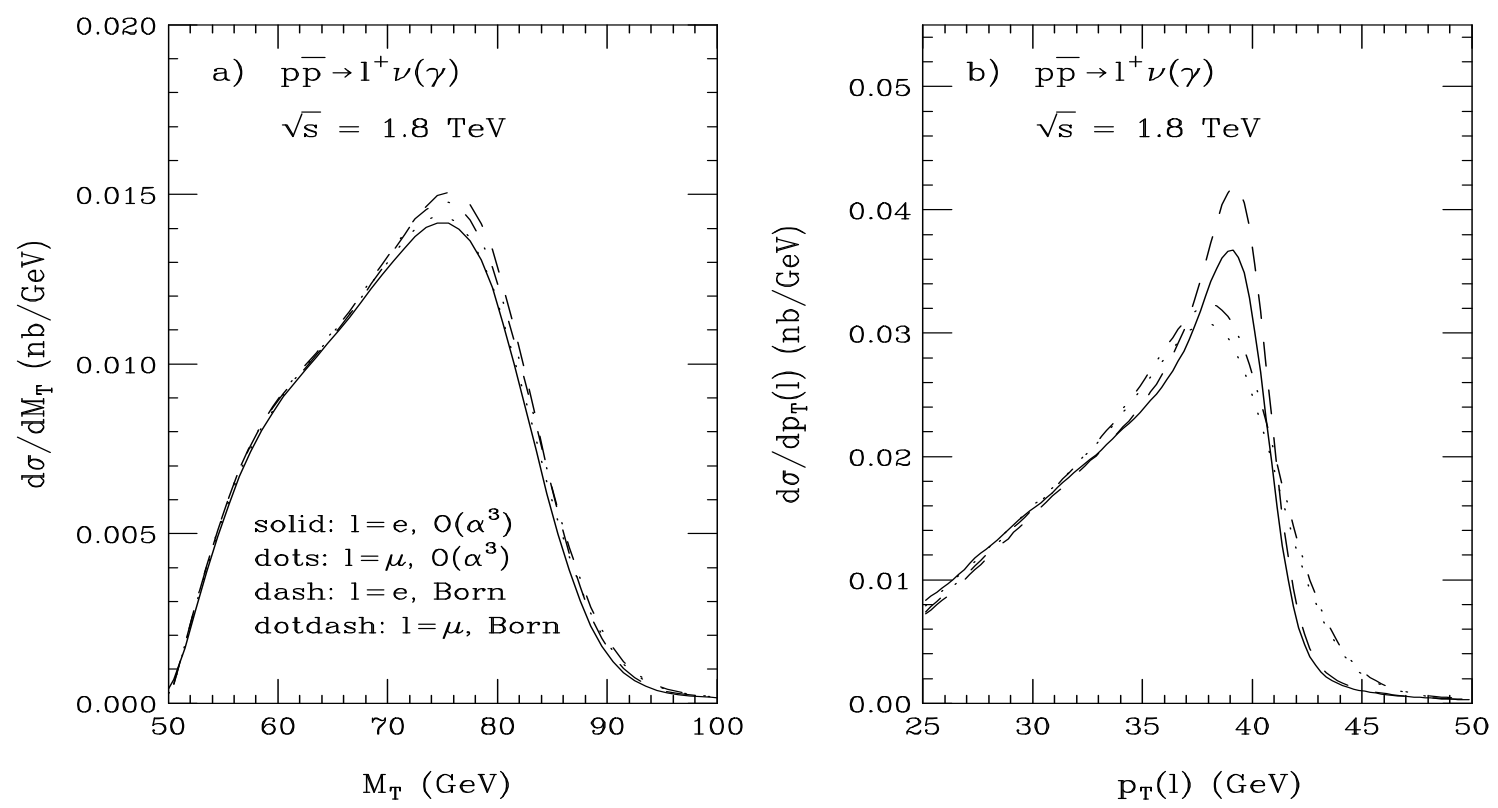

Figure 4.1: Effect of $\mathcal{O}(\alpha)$ electroweak corrections on $M_{T}^{W}$ and $p_{T}^{l}$ distributions. In both cases, the height of the Jacobian peak is reduced. Figures taken from [16].

to perform tests of generator level processes, i.e. we make Monte Carlo to Monte Carlo comparisons. This technique is used throughout the $W$ mass analysis to gauge various systematic uncertainties.

In this particular case, we input generator-level WGRAD at leading order (LO) to the fast simulation to construct a series of templates between 80 and $81 \mathrm{GeV} / c^{2}$. Then, in place of an actual data file we input a simulated data file consisting of generator-level WGRAD with an input $W$ mass of $80.450 \mathrm{GeV} / c^{2}$ with some QED effects included, e.g. FSR, ISR, etc. We also perform a fit using a LO simulated data file. This provides a baseline for measuring the shifts from the other processes. We find this fit comes out consistent with the input $W$ mass of $80.450 \mathrm{GeV} / c^{2}$ at the $5 \mathrm{MeV} / c^{2}$ level, indicating the fitter and simulation are unbiased at this level. Based on this we attribute a $5 \mathrm{MeV} / c^{2}$ systematic uncertainty to the simulation and fitter. We then observe the difference between the fitted $W$ mass and the baseline value to determine the impact of the process in question. 
It should be noted that WGRAD doesn't provide a realistic description of $W$ events since it produces $W$ bosons with no transverse momentum. However, it is sufficient for testing the impact of QED alone, assuming the QED effects can be decoupled from the QCD. We find in section 4.3 that this is true for the $p_{T}$ regime we are interested in.

We find shifts on the order of $5 \mathrm{MeV} / c^{2}$ from the baseline values when using ISR-only and interference-only data (see Table 4.1 for all fit results).

\begin{tabular}{|c|c|c|c|}
\hline Process & Fit Type & Shift $(\mu$ channel $)\left(\mathrm{MeV} / c^{2}\right)$ & Shift $\left(e\right.$ channel) $\left(\mathrm{MeV} / c^{2}\right)$ \\
\hline \multirow{3}{*}{$\mathrm{LO}$} & $p_{T}$ & $-7 \pm 3$ & $-4 \pm 3$ \\
\hline & $M_{T}^{W}$ & $-9 \pm 5$ & $-2 \pm 3$ \\
\hline & $\not \dot{H}_{T}$ & $-12 \pm 6$ & $-2 \pm 5$ \\
\hline \multirow{3}{*}{ Interference } & $p_{T}$ & $-4 \pm 2$ & $-7 \pm 2$ \\
\hline & $M_{T}^{W}$ & $-4 \pm 3$ & $-4 \pm 3$ \\
\hline & $E_{T}$ & $-2 \pm 6$ & $-7 \pm 5$ \\
\hline \multirow{3}{*}{ ISR only } & $p_{T}$ & $+1 \pm 3$ & $+3 \pm 2$ \\
\hline & $M_{T}^{W}$ & $-2 \pm 5$ & $4 \pm 3$ \\
\hline & $E_{T}$ & $+6 \pm 7$ & $+3 \pm 4$ \\
\hline \multirow{3}{*}{$\mathrm{FSR}(\mathrm{BK})$} & $p_{T}$ & $-127 \pm 5$ & $-87 \pm 5$ \\
\hline & $M_{T}^{W}$ & $-149 \pm 6$ & $-107 \pm 4$ \\
\hline & $E_{T}$ & $-94 \pm 8$ & $-54 \pm 7$ \\
\hline \multirow{3}{*}{ FSR } & $p_{T}$ & $-127 \pm 3$ & $-72 \pm 3$ \\
\hline & $M_{T}^{W}$ & $-152 \pm 5$ & $-82 \pm 3$ \\
\hline & $\not H_{T}$ & $-92 \pm 7$ & $-24 \pm 5$ \\
\hline
\end{tabular}

Table 4.1: Shifts in the $W$ mass due to various QED processes. ISR and interference show little effect on the fitted mass. FSR causes large shifts to lower mass values. The LO fit provides a consistency check of the fast simulation. 
For these sets of simulated data, we varied the WGRAD parameter $\delta_{s}$ over a wide range $\left(10^{-2}\right.$ to $\left.10^{-4}\right)$ and found no significant changes in the magnitude of the mass shifts. Based on these findings we attribute a $5 \mathrm{MeV} / c^{2}$ systematic uncertainty from ISR and interference. In addition, since ISR and interference are small effects over wide ranges of $\delta_{s}$, and given that FSR is the dominant effect with respect to the fitted $W$ mass, we use only FSR in our implementation of WGRAD. Note that the Berends and Kleiss (BK) approximation of FSR [18, 22], which is included as an option of WGRAD, is sufficient for studying FSR systematic effects [23]. In the case of FSR and FSR (BK), shifts to lower best-fit $W$ mass values are observed due to the changes in the lineshapes of the fit variables from the radiated photon. The shift is smaller for the electron channel since collinear photons get merged with the electron energy cluster. In general, the $\mathscr{H}_{T}$ fit has smaller shifts than the $p_{T}$ or $M_{T}^{W}$ fits because wide-angle photons are added to the hadronic recoil and thus included in the $\not_{T}$ calculation.

Having isolated FSR as the only QED process to include in our $W$ mass fits, we now investigate various systematic uncertainties potentially associated with it. One such possibility is the relation between the FSR shift and the $\delta_{s}$ parameter. Since event generation for WGRAD FSR is very CPU intensive, we use FSR (BK) to investigate the systematic uncertainty due to $\delta_{s}$. Figure 4.2 shows the variation in the shift as a function of $\delta_{s}$ for both muons and electrons. We fit a line to the results of fits for reasonable values of $\delta_{s}$ (below 0.001) and take the uncertainty on the constant term as the systematic uncertainty from the choice of $\delta_{s}$. Results are shown in Table 4.2. Note that we take $\delta_{s}=0.0001$ as the default value when generating QED photons for the mass measurement. We found that this value improved the agreement between data and $\mathrm{MC}$ in the right shoulder of the dilepton mass distribution of $Z$ candidates compared to a value of 0.001 . 

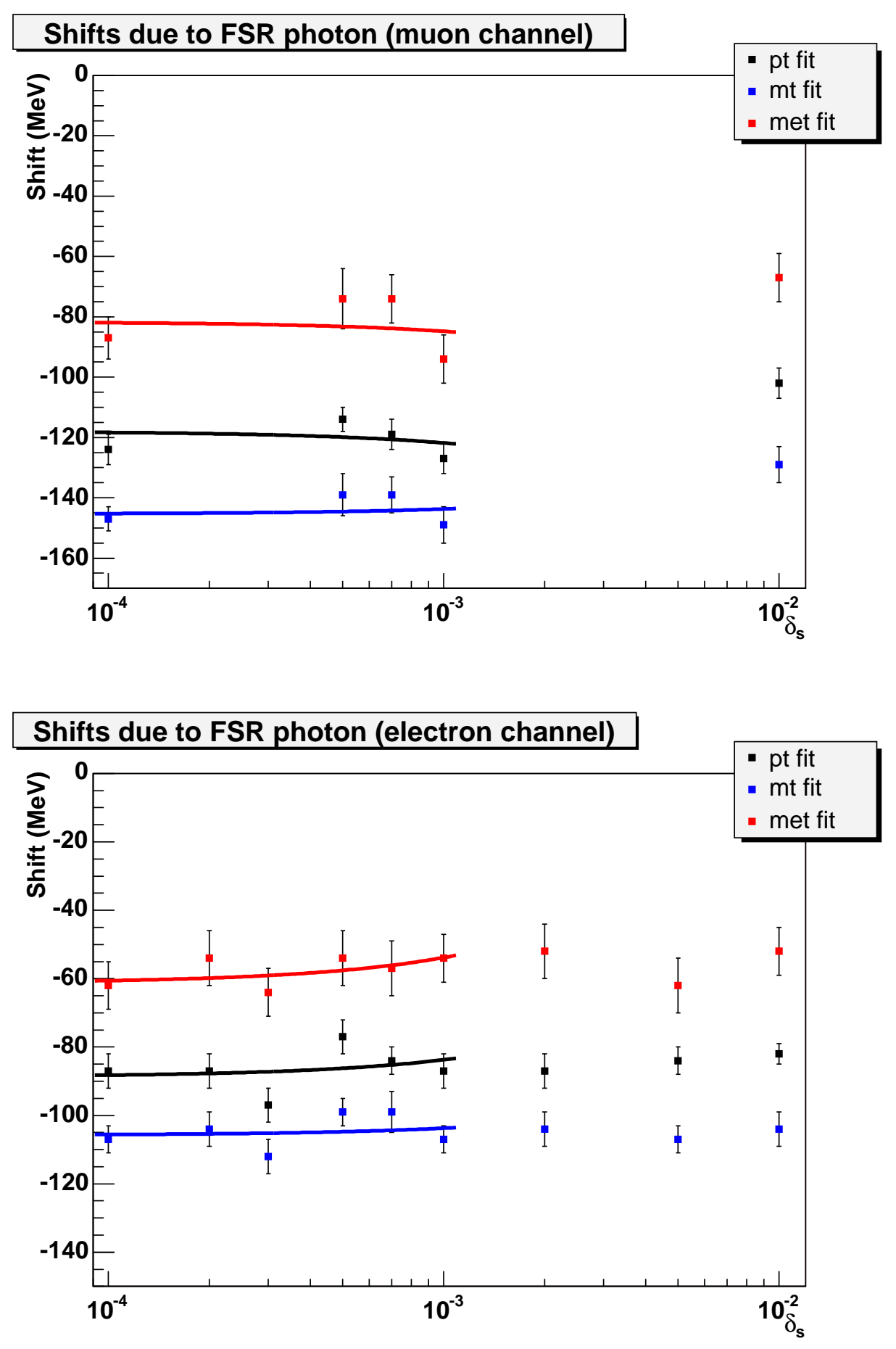

Figure 4.2: Shifts in the $W$ mass as a function of $\delta_{s}$ for the three fit variables: $p_{T}$, $M_{T}^{W}$, and $p_{T}^{\nu}$. Muons are shown on the top plot, electrons on the bottom. We take the uncertainty on the $y$-intercept as the systematic uncertainty arising from $\delta_{s}$. 


\begin{tabular}{|c|c|c|c|}
\cline { 2 - 4 } \multicolumn{1}{c|}{} & \multicolumn{3}{c|}{ Uncertainty $\left(\mathrm{MeV} / c^{2}\right)$} \\
\hline Process & $p_{T}$ & $M_{T}^{W}$ & $\mathscr{H}_{T}$ \\
\hline$W \rightarrow e \nu$ & 4 & 3 & 5 \\
\hline$W \rightarrow \mu \nu$ & 5 & 4 & 7 \\
\hline
\end{tabular}

Table 4.2: Systematic uncertainties due to $\delta_{s}$. We estimate the systematic uncertainty from $\delta_{s}$ using the error on the constant term of the fits shown in Figure 4.2.

\subsubsection{Including QED Effects in the Fit Templates}

The final goal of using WGRAD is to combine its QED corrections to $W$ decay with the fit templates generated by RESBOS. We accomplish this by constructing a histogram of photon kinematics as determined by WGRAD. We then randomly sample this histogram in the fast simulation to simulate the radiation of an FSR photon. A single photon is selected and the kinematics of the charged lepton from RESBOS are adjusted accordingly, as if it had radiated the photon. The photon is subsequently propagated through the detector volume.

The histogram we sample is a two-dimensional histogram consisting of $\sqrt[3]{y}$ vs. $\sqrt{\Delta R}$, where $y=E_{\gamma} /\left(E_{\gamma}+E_{l}\right)$ and $\Delta R(l, \gamma)=\sqrt{(\Delta \eta)^{2}+(\Delta \phi)^{2}}$. The cube- and squareroots flatten the sharply-peaking distributions of $y$ and $\Delta R$, respectively, rendering them amenable to sampling. Figures 4.3 and 4.4 show the projections of these two variables for both muons and electrons.

To quantify the systematic uncertainty introduced by this method, we constructed templates and a baseline data fit using LO WGRAD with the sampling of an FSR histogram generated at $M_{W}=80.450 \mathrm{GeV} / c^{2}$. This gives us a set of templates with QED included. We then used the original FSR events that were used to make the histogram for sampling as the input "data". If sampling the histogram exactly reproduced FSR effects, we should see no shift in the best-fit mass value from the baseline value. The 


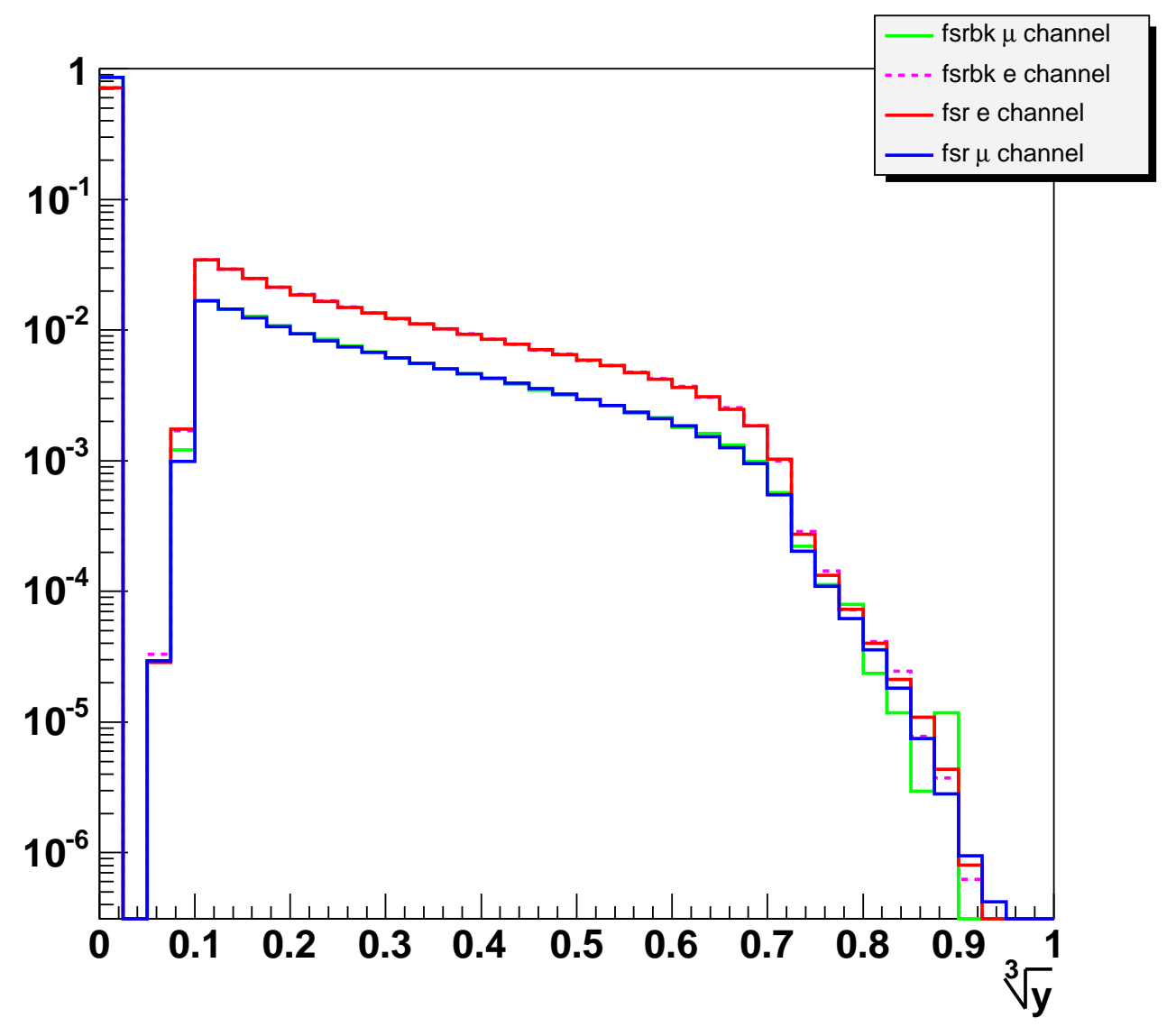

Figure 4.3: Projection of $\sqrt[3]{y}$ for muons and electrons. More photons are produced by WGRAD for electrons than for muons since there is more radiation collinear with the electron than the muon. The muon sample contains $17 \%$ events with a photon while the electron sample contains $31 \%$ with a photon. Events with no photon are in the zero bin. 


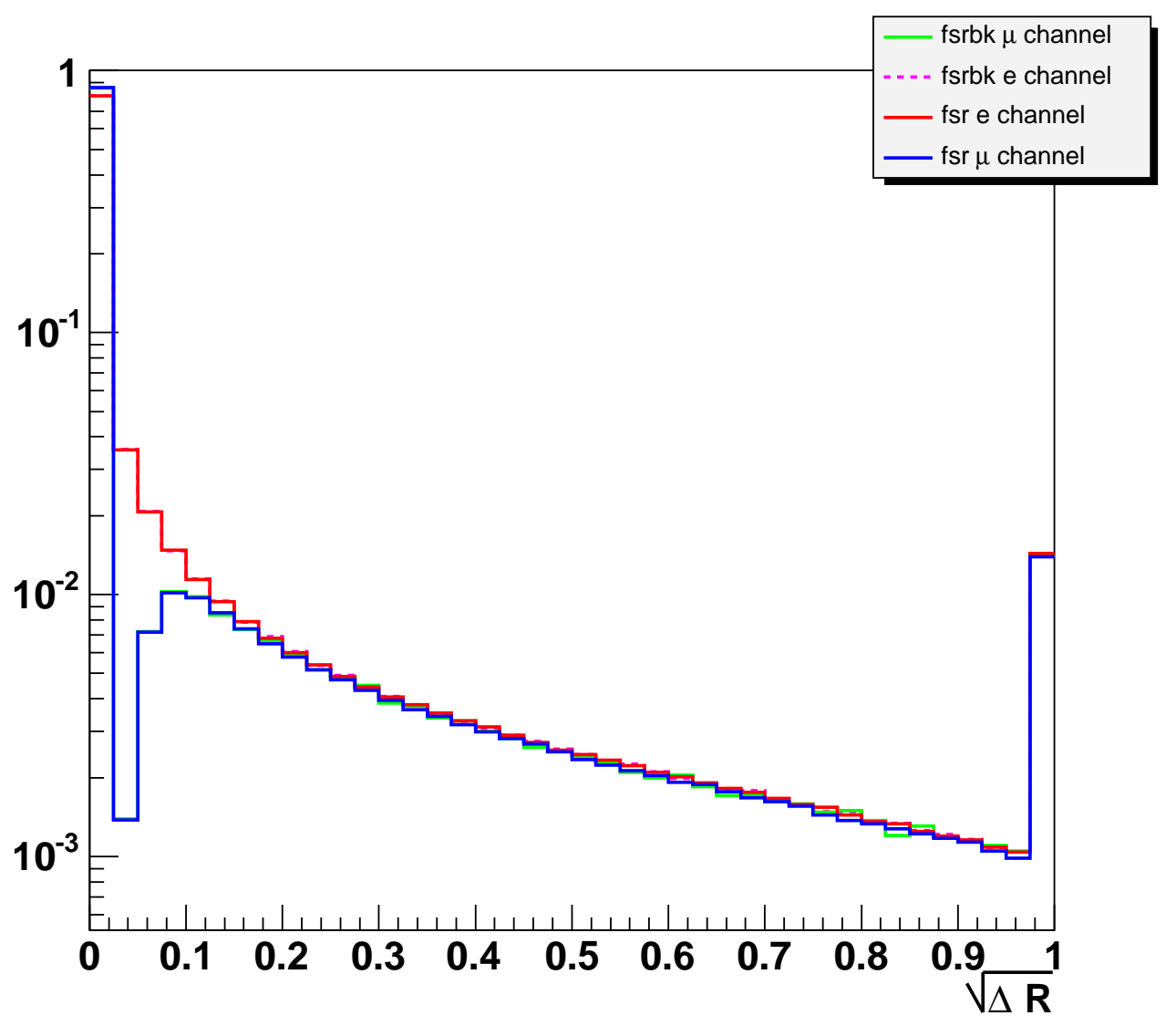

Figure 4.4: Projection of $\sqrt{\Delta R}$ for muons and electrons. Here we can see that the electrons contain significantly more collinear photons than the muons. The wide angle radiation is nearly identical between the two. Events with no photon are in the zero bin. Events with $\Delta R>1$ are in the far right hand bin. 


\begin{tabular}{|c|c|c|c|}
\cline { 2 - 4 } \multicolumn{1}{c|}{} & \multicolumn{3}{c|}{ Shift $\left(\mathrm{MeV} / c^{2}\right)$} \\
\hline Process & $p_{T}$ & $M_{T}^{W}$ & $\not_{T}$ \\
\hline$W \rightarrow e \nu$ & $-2 \pm 2$ & $+1 \pm 3$ & $+3 \pm 4$ \\
\hline$W \rightarrow \mu \nu$ & $+3 \pm 6$ & $+1 \pm 9$ & $-4 \pm 13$ \\
\hline
\end{tabular}

Table 4.3: Shifts in the $W$ mass due to sampling a histogram that contains WGRAD final state photons.

results are summarized in Table 4.3. We see no statistically significant shifts and thus do not attribute any systematic uncertainty to this method above the $5 \mathrm{MeV} / \mathrm{c}^{2}$ already included for the simulation and fitter.

\subsubsection{Second Photon}

The WGRAD calculation currently does not include a second final-state photon. Previous $W$ mass measurements [17] used the program PHOTOS to investigate the impact of a second photon. That analysis concluded that the effect of a second photon on $M_{W}$ (for the transverse mass fit) was less than $10 \mathrm{MeV} / c^{2}$ for the muon channel and less than 5 $\mathrm{MeV} / c^{2}$ for the electron channel [17]. The authors of WGRAD are currently working on a calculation that includes the effect of a second photon [23] and we intend to incorporate this when it becomes available. However, for now we assume the shift in the fitted $W$ mass from a second photon is $10 \%$ of the single photon shift [24], and take this as a systematic uncertainty on the measured mass. These values are consistent with those obtained by sampling the QED histograms twice to simulate the effect of a second photon.

\subsubsection{QED Radiation for $Z \rightarrow l l$}

In constructing fitting templates for the $Z$ mass we also need to include QED corrections similar to those for the $W$ mass. We do this by sampling a histogram of $\sqrt[3]{y}$ vs. $\sqrt{\Delta R}$ 
generated with WGRAD with the input $W$ mass set to the $Z$ mass, $91.188 \mathrm{GeV} / c^{2}$. By using the $Z$ mass value we account for $Q^{2}$ effects that influence $\sqrt[3]{y}$ vs. $\sqrt{\Delta R}$. The motivation for using WGRAD instead of ZGRAD is that in ZGRAD there is an ambiguity as to which charged lepton radiated the photon, since either $Z$ leg can radiate. In WGRAD, there is no such ambiguity. We sample the $Q^{2}$-scaled WGRAD histogram for a single $Z$ leg twice in an attempt to model radiation of the $Z$ decay products. Shifts in fitted $Z$ mass are shown in Table 4.4. Since this is an approximate solution, we use an unweighted version of ZGRAD described in [25] as a cross-check of the procedure. We find the magnitudes of the shifts due to FSR are consistent between the two.

We also observe that the $Q^{2}$-scaling made a noticeable improvement between data and Monte Carlo in the shoulder of the $Z$ invariant mass distribution.

\begin{tabular}{|c|c|c|c|}
\hline Process & Fit Type & Shift $(\mu$ channel $)\left(\mathrm{MeV} / c^{2}\right)$ & Shift $(e$ channel $)\left(\mathrm{MeV} / c^{2}\right)$ \\
\hline LO & $M_{Z}$ & $-5 \pm 4$ & $-1 \pm 5$ \\
\hline FSR & $M_{Z}$ & $-220 \pm 4$ & $-198 \pm 6$ \\
\hline
\end{tabular}

Table 4.4: Shifts in the fitted $Z$ mass for LO and FSR via sampling a $Q^{2}$-scaled WGRAD histogram. Note that the LO entry indicates that the $Z$ simulation and fitter are unbiased at the $\sim 5 \mathrm{MeV} / c^{2}$ level.

All the QED systematic uncertainties on the $W$ mass are shown in Table 4.5. We take the values to be the same for all fit types $\left(p_{T}, M_{T}^{W}\right.$, and $\left.\mathbb{E}_{T}\right)$.

\subsection{QCD with RESBOS}

The $W p_{T}$ spectrum arises from QCD processes such as gluon radiation and has a large impact on the lineshapes of the fit variables $M_{T}^{W}, p_{T}^{l}, \not_{T}$. In general, the more boosted the $W$ boson is, the greater the kinematic distributions of the decay products will be distorted. The boson $p_{T}$ is particularly important for the $p_{T}^{l}$ fit since it is directly 


\begin{tabular}{|c|c|c|c|}
\cline { 2 - 4 } \multicolumn{1}{c|}{} & \multicolumn{3}{c|}{ Systematic $\left(\mathrm{MeV} / c^{2}\right)$} \\
\hline Source & Muon & Electron & Common \\
\hline Simulation/Fitter & 5 & 5 & 5 \\
\hline ISR/Interference & 5 & 5 & 5 \\
\hline$\delta_{s}$ & 5 & 5 & - \\
\hline Second photon & 15 & 10 & 10 \\
\hline Total QED & 17 & 13 & 12 \\
\hline
\end{tabular}

Table 4.5: Summary of QED and fitter systematic uncertainties for the mass fit in the muon and electron channels. We take the uncertainties to be the same for the three fit distributions.

influenced by the boost of the $W$.

We model the $W p_{T}$ spectrum using RESBOS, which computes the quintuple differential cross section $\frac{d^{5} \sigma}{d Q_{T}^{W} d y_{W} d Q_{W}^{2} d \Omega}$ for $p \bar{p} \rightarrow W^{ \pm}$. Exact matrix elements for $W$ production and decay are used. NLO and soft-gluon resummed initial state QCD corrections are included, the latter of which are the dominant contribution to the cross section at low $p_{T}$. For the soft-gluon resummation the Collins-Soper-Sternman (CSS) [15] formalism is used, in which all leading and large logs are resummed. Physically, resummation corresponds to summing over the most important contributions from multiple gluon emission [10]. In RESBOS, the final state is given by one massless charged lepton and one massless uncharged lepton, i.e. no distinction is made between electrons or muons in the final state. Note that RESBOS doesn't contain any QED radiative corrections.

The structure of the Monte Carlo event generator is based on that of PAPAGENO [26] and uses VEGAS as the phase space integration routine. RESBOS is superior to other event generators in that it models the $W p_{T}$ spectrum well at low $p_{T}$ via multiplesoft-gluon resummation techniques, and it contains several of the most important helicity amplitudes for $W$ production. The helicity amplitudes influence the angular distributions 
of the final-state leptons at higher $p_{T}$.

\subsection{1 $W$ Boson Generation}

We use RESBOS to generate large samples $\left(47 \times 10^{6}\right)$ of $W$ events at $M_{W}=80 \mathrm{GeV} / c^{2}$ and $M_{W}=81 \mathrm{GeV} / c^{2}$ that are used for constructing the fit templates. The high statistics of these Monte Carlo samples ensures any statistical uncertainty introduced by the fitting templates is negligible compared to the statistical uncertainty of the data sample. The $W$ width used is fixed at $2.12 \mathrm{GeV}[7]$. These samples have been validated by fitting the invariant mass spectrum for the pole mass and width using a relativistic Breit-Wigner. The fit values for both the mass and width are consistent with the input values. In addition, further validation is performed using the fast simulation in that the fit value from a $\mathrm{MC}$ data file (also with $47 \times 10^{6}$ events) is consistent with the input invariant mass (at the $5 \mathrm{MeV} / c^{2}$ level).

As a check of the helicity amplitudes used in RESBOS, we use a procedure described in [27] to extract the angular information from the Monte Carlo samples. In short, this process consists of computing moments of the distribution, which are directly related to the helicity amplitudes. We compare the results from RESBOS to another QCD event generator, DYRAD [28]. We find reasonable agreement between the two, see Figure 4.5. We investigate the impact of varying the coefficients (via re-weighting the events) over wide ranges and find no significant impact on the mass measurement.

\subsection{2 $Z$ Boson Generation}

In analogous fashion to the generation of $W$ bosons, we generate large samples $\left(31 \times 10^{6}\right)$ of $Z$ events with RESBOS. We generate events using a $Z$ width of $2.5 \mathrm{GeV}$ [7].

We carry out the same validation procedure of these samples as for the $W$ samples. We fit the invariant mass spectrum of the $Z$ bosons with a relativistic Breit-Wigner 


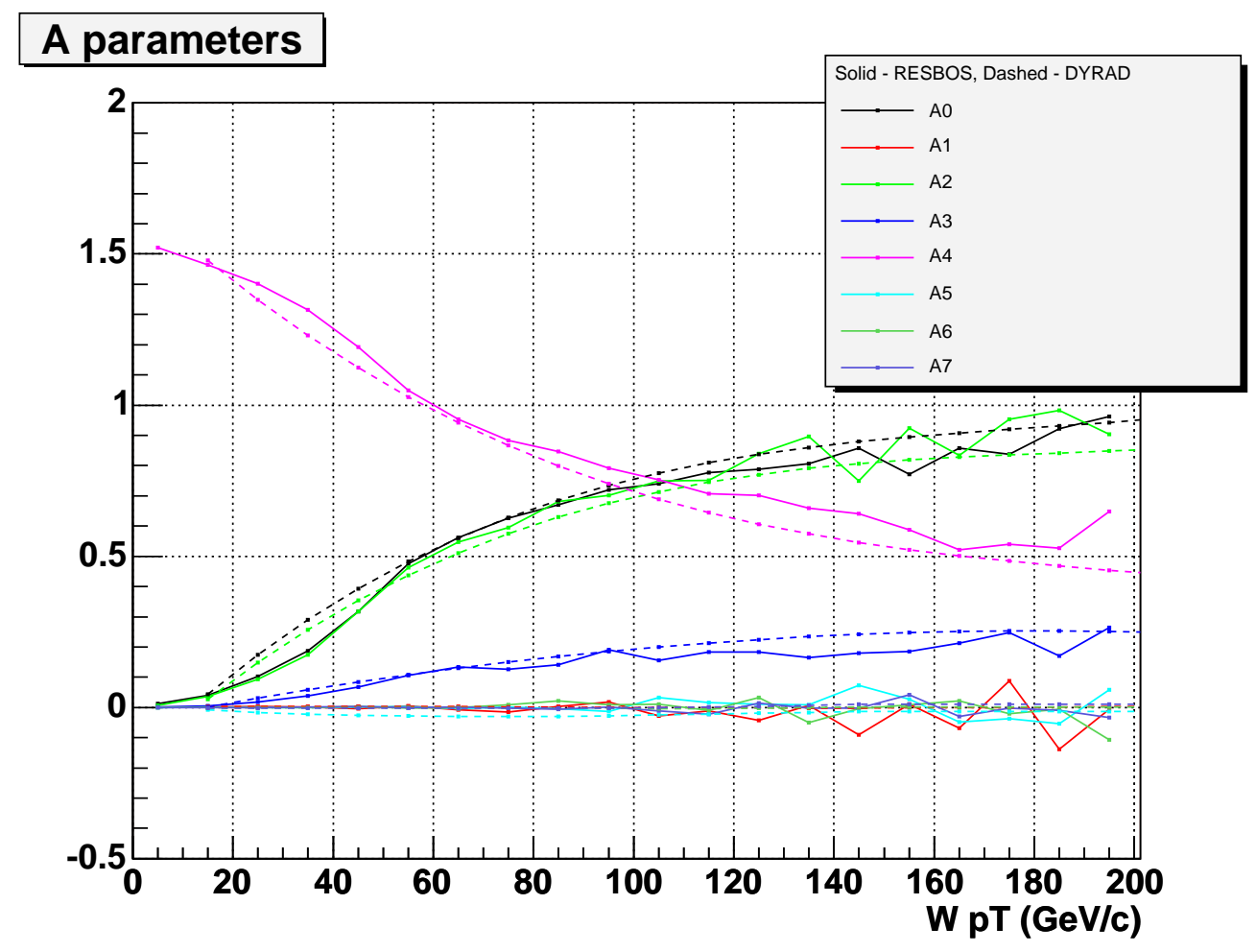

Figure 4.5: Helicity amplitudes from RESBOS and DYRAD. The solid lines are RESBOS and the dashed lines are DYRAD.

distribution given by,

$$
\mathcal{L}_{q \bar{q}}(Q) \frac{Q^{2}}{\left(Q^{2}-M_{Z}^{2}\right)^{2}+Q^{4} \Gamma_{Z}^{2} / M_{Z}^{2}}
$$

where $\mathcal{L}_{q \bar{q}}(Q)=e^{-\beta Q} / Q$, is the parton-luminosity skewing term, which accounts for the decrease in parton-luminosity with increasing mass. The parameter $\beta$ is referred to as the parton-luminosity slope. From our fit we extract a value of $\beta=3.56 \times 10^{-3} \mathrm{GeV}^{-1}$, which is consistent with the value quoted in [29].

Standard $Z$ generation with RESBOS does not include the Drell-Yan and $Z / \gamma$ interference contributions. These contributions need to be generated separately and then combined with the $Z$-pole distribution. Since these contributions are small we chose to include them at the fitting stage as if they were backgrounds to $Z$ production. The 
distributions are shown in Figure 4.6 and the normalizations are shown in Table 4.6. The normalizations are obtained by taking the ratio of the integral of the "background" distribution to the integral of the $Z$-pole distribution over the region $70-110 \mathrm{GeV} / c^{2}$ for each contribution. The uncertainties on the normalizations are negligible due to the high statistics of the samples used.

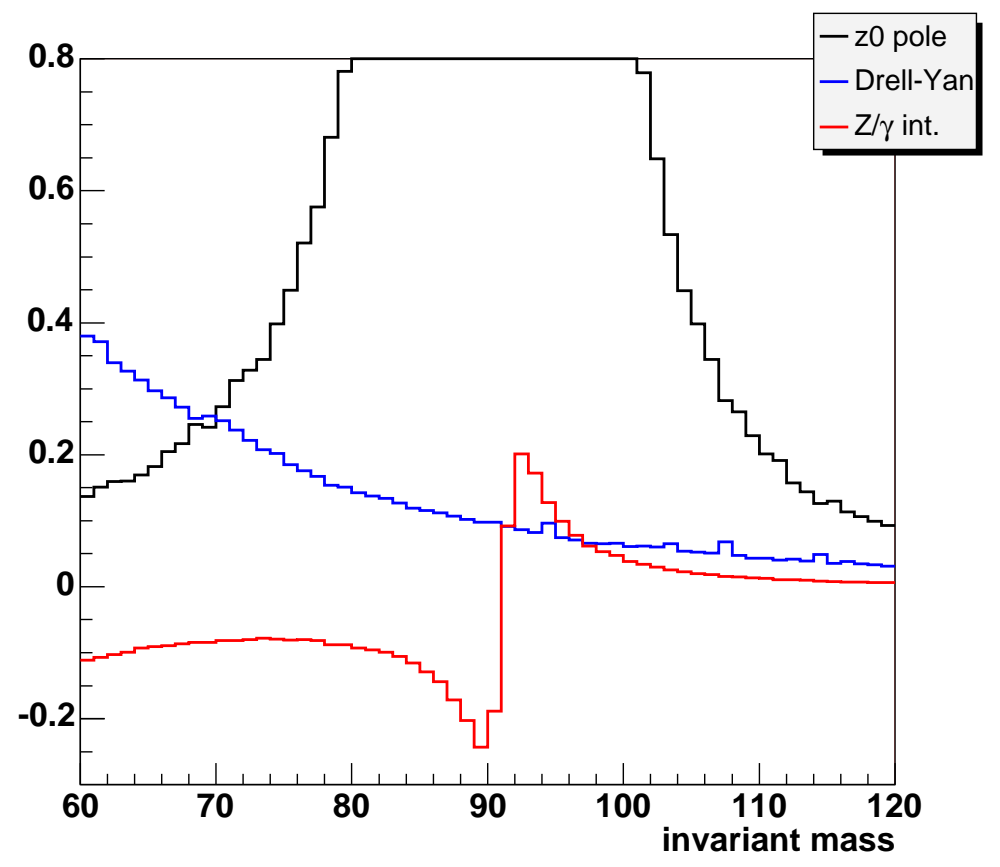

Figure 4.6: Drell-Yan and $Z / \gamma$ interference contributions to $Z$ production from RESBOS. The interference contribution changes sign at the $Z$ pole while the Drell-Yan steadily decreases with increasing mass. For comparison, the $Z$-pole distribution is (partially) shown.

\subsubsection{Impact of QCD Processes on the $W$ Mass}

Similar to the QED corrections discussed in section 4.1.1, we are primarily interested in the effect of NLO QCD and resummation on the $M_{T}^{W}$ and $p_{T}^{l}$ distributions. Figure 4.7 


\begin{tabular}{|c|c|}
\hline Process & Normalization \\
\hline$\gamma$-pole & 0.023 \\
\hline$Z / \gamma$ Int. & 0.005 \\
\hline
\end{tabular}

Table 4.6: Normalization for the additional contributions to the $Z$ lineshape of the $\gamma$ pole and $Z / \gamma$ interference. The statistical uncertainties are negligible. These process are treated like backgrounds to $Z$ production.
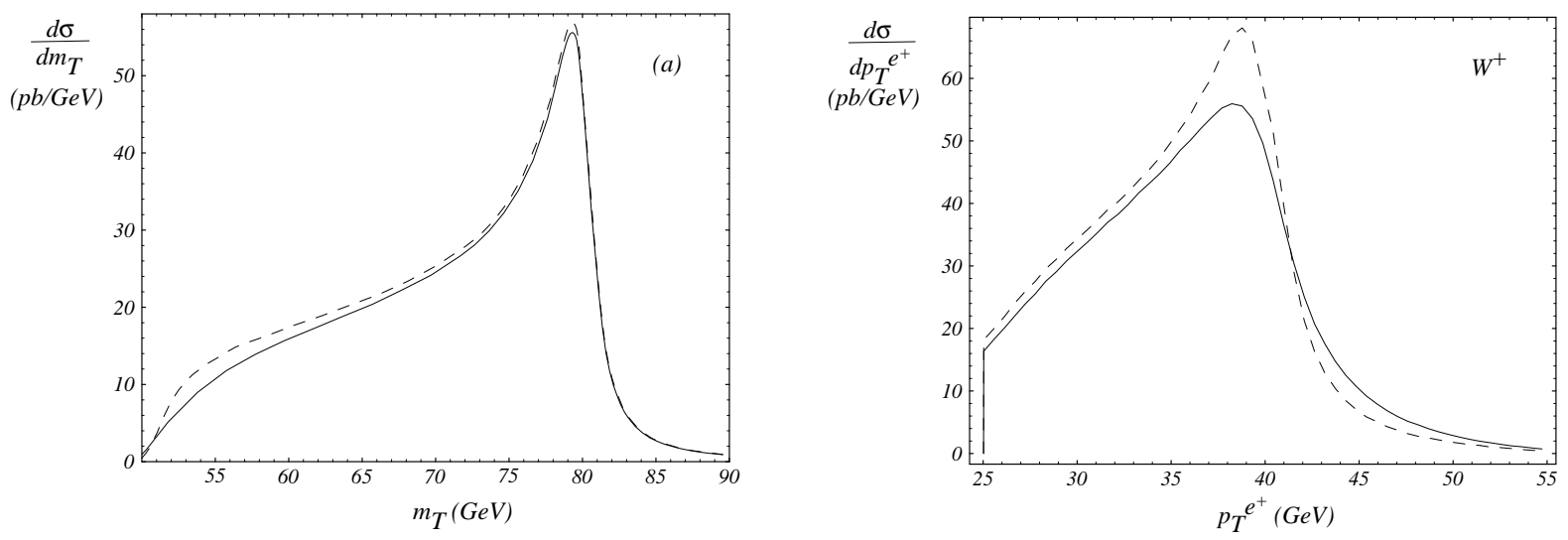

Figure 4.7: Comparison of NLO QCD with NLO QCD plus resummation in the $M_{T}^{W}$ and $p_{T}^{l}$ distributions taken from [15]. The dashed curve is for NLO QCD and the solid line is for NLO QCD including resummation.

shows the $M_{T}^{W}$ and $p_{T}^{l}$ distributions for NLO QCD and NLO QCD with resummation using RESBOS. The Jacobian peak in the $M_{T}^{W}$ distribution is broadened and smoothed by resummation [15]. In addition, although it can't be seen in the figure, the right shoulder of the distribution is shifted by approximately $50 \mathrm{MeV} / \mathrm{c}^{2}$. The effects are small since $M_{T}^{W}$ is rather insensitive to higher order QCD corrections. The $p_{T}^{l}$ distribution, on the other hand, receives a large correction and a more pronounced broadening and smoothing occurs. 


\subsubsection{Systematic Uncertainties from RESBOS}

In RESBOS, the production cross section is given by the partonic cross section convoluted with the parton distribution functions. In the momentum-space calculation of the partonic cross section, the non-perturbative physics is described by three parameters that must be determined from experiment. Conventionally these parameters are called $g_{1}, g_{2}$, and $g_{3}$. One particular choice for parameterizing the non-perturbative physics is with $S$, given by

$$
S=\left[g_{1}+g_{2} \ln \left(\frac{Q}{2 Q_{0}}\right)\right] b^{2}+g_{1} g_{3} \ln \left(100 x_{1} x_{2}\right) b
$$

where $Q_{0}$ is a fixed cutoff parameter, $b$ is the impact parameter in momentum space, and $x_{1}$ and $x_{2}$ are the momentum fractions of the initial state partons [29].

The other free parameter in the model is $\Lambda_{Q C D}$. However, since $\Lambda_{Q C D}$ is fixed by the choice of PDF we focus solely on the impact of the parameters $g_{1}, g_{2}$, and $g_{3}$. Previous analyses have shown that the dominant effects on the $W p_{T}$ spectrum come from changes in $g_{2}$ and $\Lambda_{Q C D}$ [30], where increases in $g_{2}$ tend to increase the mean boson $p_{T}$, see Figure 4.8 .

To determine the systematic uncertainty from modelling the $W p_{T}$ spectrum, we first construct templates with RESBOS using parameter values obtained from a global fit to low-energy Drell-Yan and Tevatron Run 1 Z data [31]:

$$
\begin{aligned}
& g_{1}=0.21 \mathrm{GeV}^{2} \\
& g_{2}=0.68 \mathrm{GeV}^{2} \\
& g_{3}=-0.60
\end{aligned}
$$

We then use simulated data events generated with $M_{W}=80.450 \mathrm{GeV} / c^{2}$ and various $g$ parameters. We take the $W p_{T}$ systematic corresponding to a $g_{2}$ variation of $0.12 \mathrm{GeV}^{2}$ 


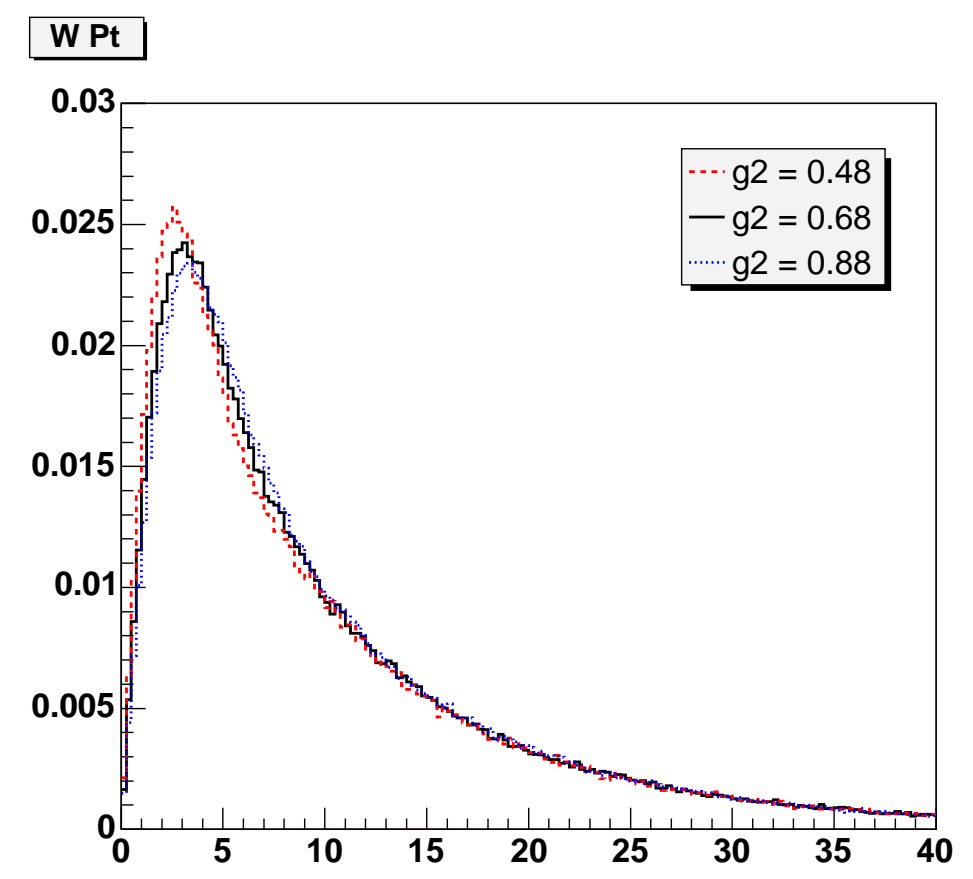

Figure 4.8: $W p_{T}$ spectrum generated by RESBOS for various $g_{2}$ values. The mean $W p_{T}$ increases by approximately $150 \mathrm{MeV} / c$ for each increase of $0.2 \mathrm{GeV}^{2}$ in $g_{2}$.

leaving $g_{1}$ fixed [29]. $g_{1}$ and $g_{2}$ are $100 \%$ anti-correlated at a fixed Q value, so varying $g_{2}$ by some amount is equivalent to varying $g_{1}$ by the same amount. In addition, following [32] we vary $g_{3}$ by \pm 0.3 . The systematic uncertainties extracted from these variations are shown in Table 4.7 .

\subsection{QED/QCD Correlations}

We have investigated QED/QCD correlations with the program RESBOS-A [33], which is a Monte Carlo event generator similar to standard RESBOS, however, includes the full calculation of a single final state photon. We believe this program will be invaluable for investigating QED/QCD correlations and may even be sufficient as the sole source for generating $W$ and $Z$ events for the $W$ mass analysis. However, since we are still in the 


\begin{tabular}{|c|c|c|c|}
\cline { 2 - 4 } \multicolumn{1}{c|}{} & \multicolumn{3}{c|}{ Systematic $\left(\mathrm{MeV} / c^{2}\right)$} \\
\hline Parameter Range & $p_{T}$ & $M_{T}^{W}$ & $\not_{T}$ \\
\hline$g_{2} \pm 0.12 \mathrm{GeV}^{2}$ & 22 & 7 & 11 \\
\hline$g_{3} \pm 0.3$ & 16 & 11 & 14 \\
\hline \hline Total QCD $/ W p_{T}$ & 27 & 13 & 18 \\
\hline
\end{tabular}

Table 4.7: Systematic uncertainties due to varying the $g$ parameters in RESBOS about their nominal values. We combine muons and electrons and take the uncertainties to be common to both. The numbers include a statistical uncertainty of $\sim 4 \mathrm{MeV} / c^{2}$.

process of validating the program, which itself is still evolving, we chose not to use it as the primary source of $W$ mass event generation.

As an estimate of the impact of $\mathrm{QED} / \mathrm{QCD}$ correlations we generated two-dimensional WGRAD FSR histograms with various constant transverse boosts applied to the $W$ boson. This roughly simulates the differences we might see if we used a MC generator that had both a non-zero $W p_{T}$ spectrum and final state photon radiation. Since the $Z$ mass fit value is more sensitive to QED radiation than the $W$ fit values, we chose to evaluate the impact of boosting with the $Z$ mass distribution. The templates are LO RESBOS $\mathrm{Z}$ events and the MC data is LO RESBOS $\mathrm{Z}$ events with sampling of a WGRAD histogram with various boosts applied. The results for various boosts are shown in Table 4.8. We can see only very large boosts have an impact, indicating QED/QCD correlations are small in the $p_{T}$ range of interest for this measurement $\left(p_{T}^{W}<15 \mathrm{GeV} / c\right)$.

\subsection{Parton Distribution Functions}

Each of the event generators above uses a set of PDFs that describe the fraction of momentum carried by the proton and antiproton valence quarks. The PDFs also describe the momentum of the sea quarks. In $W$ production, the imbalance of momenta in the 


\begin{tabular}{|c|c|}
\hline$p_{T}$ boost applied to WGRAD histogram $(\mathrm{GeV} / c)$ & $Z$ mass shift $\left(\mathrm{MeV} / c^{2}\right)$ \\
\hline No boost & $-250 \pm 4$ \\
\hline 10 & $-250 \pm 4$ \\
\hline 100 & $-237 \pm 4$ \\
\hline 1000 & $-69 \pm 4$ \\
\hline
\end{tabular}

Table 4.8: $Z$ mass shifts from sampling a boosted QED histogram. Note that the results shown are for the muon channel only. We can see that for small boosts the effect is negligible. It only becomes important for very large boosts $(\sim 1000 \mathrm{GeV} / c)$, which are not relevant to our measurement of the $W$ mass.

longitudinal direction as given by the PDFs causes the bosons to be boosted along the $\pm z$-axis. Therefore finite detector acceptance and kinematic cuts applied to the boson decay products cause the choice of PDFs to influence the measurement of the $W$ mass.

The PDF set we use is produced by the CTEQ collaboration from global fits to deep inelastic scattering data, jet production, and measurements of the $W$ charge asymmetry [34]. We also use a PDF set produced by the MRST collaboration as a cross-check [35].

Specifically, we use the CTEQ6M PDF set, which uses 20 free parameters in the global fit to the data. A $20 \times 20$ matrix is diagonalized and 20 orthogonal eigenvector pairs are determined. Each eigenvector pair corresponds to a linear combination of the parameters. The CTEQ collaboration has provided $20 \mathrm{PDF}$ sets that have positive and negative variations of each eigenvector such that $\Delta \chi^{2}=100$, which is intended to represent a $90 \%$ confidence limit. We use PYTHIA [36] to generate $40 \times 10^{6} \mathrm{~W}$ events for each of the 20 PDF sets. We run each of these through the fast simulation and compare the $W$ mass to that obtained with the default CTEQ6M template, see Figure 4.9.

The CTEQ collaboration provides a formula to compute the 90\% CL uncertainty, 


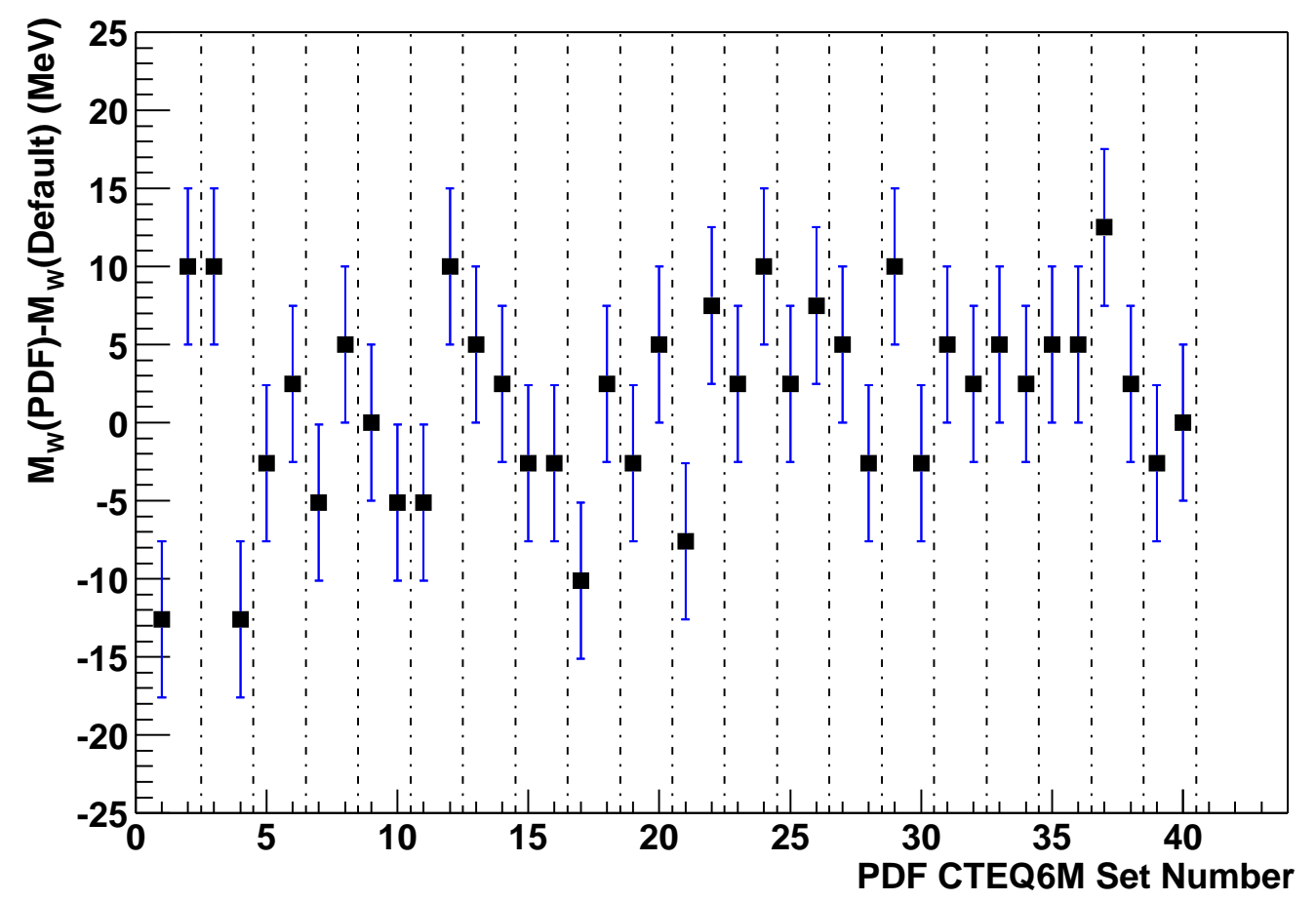

Figure 4.9: Changes in $M_{W}$ for 20 error PDFs. We take the difference in $M_{W}$ between each error PDF set and the CTEQ6M default.

$$
\delta M_{W}=\frac{1}{2} \sqrt{\sum_{i}\left(\Delta_{i} M_{W}^{+}-\Delta_{i} M_{W}^{-}\right)^{2}}
$$

Since we are interested in a $1 \sigma(68 \%)$ variation as the PDF systematic, we divide the result of equation 4.8 by 1.6 . This gives $18 \mathrm{MeV} / c^{2}$ as the systematic uncertainty on the $W$ mass due to PDFs. We follow the same procedure for the $M_{T}^{W}$ and $\not_{T}$ fits, obtaining 15 and $19 \mathrm{MeV} / c^{2}$, respectively.

As a cross-check, we generate $40 \times 10^{6}$ PYTHIA events with the MSRT2003 PDF set and compare it to the default CTEQ set, via the fast simulation. The difference in fitted masses is roughly half the size of difference obtained with the 40 error PDFs. Therefore, we assume that the CTEQ estimation of the PDF uncertainty is accurate. 


\section{Chapter 5}

\section{Event Selection and Backgrounds}

This chapter describes how we obtain the data samples used in the analysis. We go on to describe how we model the backgrounds present in the $W \rightarrow \mu \nu$ sample.

\subsection{Event Selection}

The aim in selecting the event samples for the $W$ mass measurement is to achieve high statistics samples with minimal background. For high $p_{T}$ muon events, we begin with a

dataset of $191 \mathrm{pb}^{-1}$ that was constructed using a general purpose high $p_{T}$ lepton trigger. Muon selection then proceeds by looking for the two highest $p_{T}$ COT tracks in the event. The tracks are required to pass various cuts, including checking if they are consistent with tracks from a $Z$ boson or cosmic rays. Finally, kinematic cuts are applied to maximize the signal to background ratio.

In addition to the high $p_{T}$ muon dataset of $W \rightarrow \mu \nu$ and $Z \rightarrow \mu \mu$, we also use a high statistics $J / \psi \rightarrow \mu \mu$ sample and a $\Upsilon \rightarrow \mu \mu$ sample for detector calibration. The numbers of candidate events are shown in Table 5.1.

Note that several other datasets are used indirectly in this analysis. We use a large sample of so-called minimum bias events, triggered only by the coincidence of the luminosity counters, to determine some of the recoil model parameters. Cosmic ray events are 


\begin{tabular}{|c|c|}
\hline Sample & Candidates \\
\hline$J / \psi \rightarrow \mu \mu$ & 398832 \\
\hline$\Upsilon \rightarrow \mu \mu$ & 27080 \\
\hline$Z \rightarrow \mu \mu$ & 4943 \\
\hline$W \rightarrow \mu \nu$ & 51240 \\
\hline
\end{tabular}

Table 5.1: Data samples used in the $W$ mass analysis. Numbers of candidate events are after all cuts have been applied.

used for alignment of the COT detector and validation of the amount of ionizing material in the detector volume. $W \rightarrow e \nu$ events are used to tune the number of radiation lengths in the detector simulation and to determine curvature corrections. $Z \rightarrow e e$ events are used along with $Z \rightarrow \mu \mu$ events to tune the recoil model parameters.

\subsection{1 $J / \psi \rightarrow \mu \mu$ Data Sample}

A sample of $J / \psi \rightarrow \mu \mu$ candidates is used to determine the momentum scale and to tune the amount of ionizing energy loss in the detector. This sample is triggered on di-muon candidates with the following criteria:

- COT only tracks

- 8 COT superlayers with at least 7 hits each

- $\left|d_{0}\right|<0.3 \mathrm{~cm}$

- $\left|\Delta z_{0}\right|<3 \mathrm{~cm}$

- muon $p_{T}>2 \mathrm{GeV} / c$

The $d_{0}$ cut requires the track to be within $0.3 \mathrm{~cm}$ of the beamline in the $r-\phi$ plane. The $\Delta z_{0}$ cut requires the difference between the $z$ coordinates of the tracks to be less than 3 $\mathrm{cm}$. See section 6.2 for further discussion of the track parameters $z_{0}$ and $d_{0}$. 


\subsection{2 $\Upsilon \rightarrow \mu \mu$ Data Sample}

A sample of $\Upsilon \rightarrow \mu \mu$ candidates is used to determine the momentum scale. In addition, since $\Upsilon$ mesons are only produced promptly, we can study the effect of beam-constrained vs. non beam-constrained tracks, where beam-constraining the track involves including an additional point at the beamspot in the track fit. That is, a non beam-constrained track consists of only hits in the COT, whereas a beam-constrained track has these same hits, however, also includes the beamspot in the fit. Note that we can't do this with the $J / \psi$ events since they can come from the decay of long-lived B mesons, which can travel a significant distance from the beamspot before decaying. The $\Upsilon$ events are selected using di-muon candidates with one muon having hits in the CMUP detector and the other having hits in either the CMX detector or the CMU detector. In addition, the di-muon candidates must satisfy:

- $p_{T}^{\mathrm{CMUP}}>4.2 \mathrm{GeV} / c$

- $p_{T}^{\mathrm{CMX}}$ or $p_{T}^{\mathrm{CMU}}>3.2 \mathrm{GeV} / c$

- 5 or more hits in at least 3 axial and 3 stereo COT superlayers

- $\left|\Delta z_{0}\right|<3 \mathrm{~cm}$

- $\left|d_{0}\right|<0.3 \mathrm{~cm}$

The $p_{T}$ cuts are taken to be just above the cuts of $3 \mathrm{GeV} / c(\mathrm{CMX}, \mathrm{CMU})$ and 4 $\mathrm{GeV} / c$ (CMUP) used in the trigger to avoid any bias due to the trigger threshold.

\subsubsection{High $p_{T}$ Muon Samples: $W \rightarrow \mu \nu$ and $Z \rightarrow \mu \mu$}

Since the $Z \rightarrow \mu \mu$ sample is used to set the parameters of the recoil model as well as cross-check the momentum scale, the $W$ and $Z$ event selection is chosen to be as similar as possible to avoid any bias. 
In general, the $W$ and $Z$ samples are obtained using a general purpose high $p_{T}$ muon trigger which requires muon candidates to have hits in the muon chambers (CMUP: $|\eta|<0.6$, CMX: $0.6<|\eta|<1.0)$ and a matching COT track with $p_{T}>8 \mathrm{GeV} / c$. At L3, the muon candidate is required to have $p_{T}^{\mu}>18 \mathrm{GeV} / c$.

The event selection iterates over all muon candidates in the event, first checking if the event is consistent with a $Z$ boson. The criteria for a $Z$ event is the presence of exactly two identified muons (at least one of which has an associated stub in the muon chambers) satisfying:

- $p_{T}^{\mu}>10 \mathrm{GeV} / c$

- $E_{\text {em }}<2 \mathrm{GeV}$

- $E_{\text {had }}<6 \mathrm{GeV}$

- isolation $<0.1$

- opposite charge

The cuts on hadronic and electromagnetic energy are chosen to be consistent with a minimum ionizing particle.

Isolation is defined as the proportional amount of energy surrounding an object (e.g. muon, electron, jet). Using a jet as an example, isolation is defined as,

$$
I s o=\frac{E_{\text {cone }}\left(\text { excluding } E_{\text {jet }}\right)}{E_{\text {jet }}}
$$

This says $I$ so is the energy inside a cone surrounding the jet (excluding the energy of the jet itself), divided by the energy of the jet. Generally a cone of $\Delta R=\sqrt{\Delta \phi^{2}+\Delta \eta^{2}}=0.4$ in $\eta-\phi$ space is used to define the region surrounding the jet. In the context of a muon candidate, it is the amount of energy in a cone divided by the muon $p_{T}$. 
If the event is not identified as a $Z$ boson then the selection proceeds by using an algorithm that checks if the muon is consistent with a cosmic ray [37]. If it is not a cosmic ray, then the following muon identification requirements are applied:

- Fiducial stub in the CMUP or CMX

- $E_{\text {em }}<2 \mathrm{GeV}$

- $E_{\text {had }}<6 \mathrm{GeV}$

- $|\Delta x|<3 \mathrm{~cm}(\mathrm{CMU}),<5 \mathrm{~cm}(\mathrm{CMP}),<6 \mathrm{~cm}(\mathrm{CMX})$

where $|\Delta x|$ is the distance between the extrapolated COT track and the muon stub. We can see that the selection here is similar to that for $Z$ events except that the muon candidate must have hits muon chambers, which must be reasonably close to the extrapolated track.

In addition, the following cuts are applied to the COT track:

- Track passes through all COT layers

- 5 or more hits in at least 3 stereo and 3 axial superlayers

- $\left|z_{0}\right|<60 \mathrm{~cm}$

- $\left|d_{0}\right|<0.1 \mathrm{~cm}$

- $30<p_{T}^{\mu}<55 \mathrm{GeV} / c$

- $\operatorname{Track} \chi^{2}<3$

The track $\chi^{2}$ cut requires the track to fit the COT hits well. Finally, kinematic cuts are applied to reduce background and hadronic recoil activity:

- $30<\mathscr{H}_{T}<55 \mathrm{GeV}$

- $60<M_{T}^{W}<100 \mathrm{GeV} / c^{2}$

- $u<15 \mathrm{GeV}$ 


\subsubsection{Selection Efficiencies}

The event selection process can introduce biases. In particular, we have investigated the $p_{T}$ dependence of the muon trigger efficiency, the $\eta$ dependence of the muon identification efficiency, and the dependence of the muon identification efficiency on the component of the hadronic recoil parallel to the muon $\left(u_{\|}\right)$. See section 6.4 for a more detailed discussion of $u_{\|}$.

Both the $p_{T}$ dependence of the muon trigger efficiency and the $\eta$ dependence of the muon identification efficiency are found to be flat within the kinematic region of interest.

The $u$ dependence of the muon identification efficiency is studied using the $Z \rightarrow \mu \mu$ sample, see Figure 5.1.

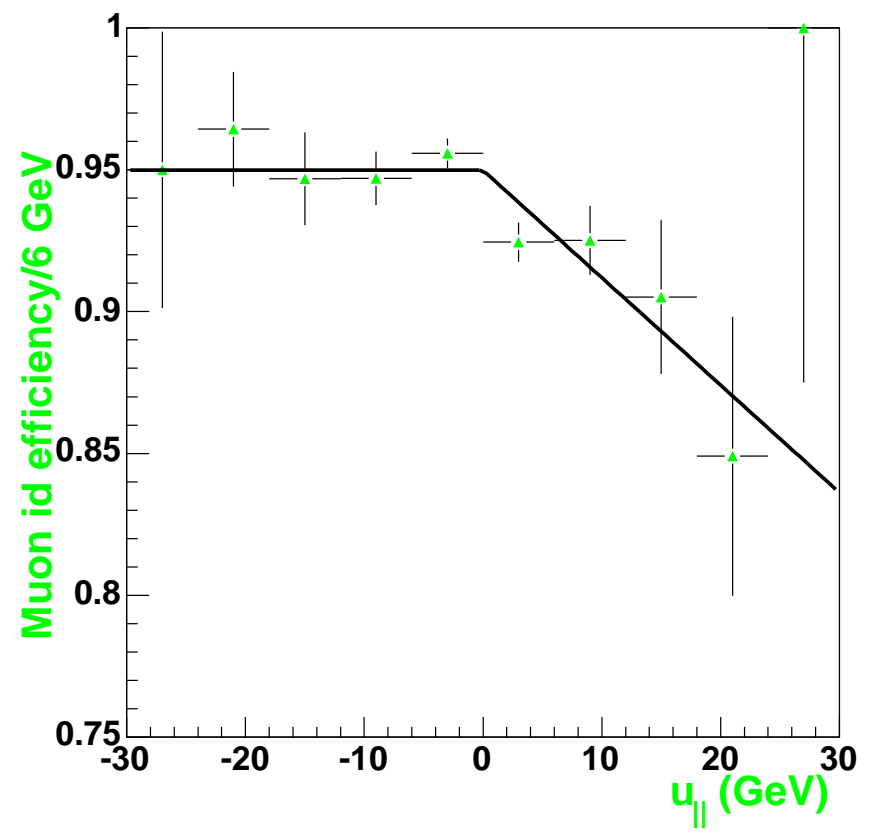

Figure 5.1: Muon identification efficiency as a function of $u_{\|}$.

We model this dependence using a linear function given by

$$
\epsilon=a\left[1-b\left(\left(u_{\|}-6\right)+\left|u_{\|}-6\right|\right)\right]
$$


where $a$ is a normalization factor and and $b$ governs the efficiency dependence on $u_{\|}$. The motivation for this particular shape is as follows: As $u_{\|}$becomes more positive (i.e. more recoil energy collinear with the muon), it becomes more difficult to identify the muon. When $u_{\|}$is small and/or opposite to the muon, we assume there is no dependence of the identification efficiency on $u_{\|}$. From a fit to the data we find,

$$
b=(-1.99 \pm 0.53) \times 10^{-3} \mathrm{GeV}^{-1}
$$

Note that the $u$ cut was increased to $30 \mathrm{GeV}$ to widen the fit range for better accuracy in the fit. The $b$ value obtained with this sample is consistent with the value obtained with the default $15 \mathrm{GeV}$ cut on $u$. To gauge the impact of the uncertainty on $b$ on the measured $W$ mass, we vary $b$ by its uncertainty and observe the change in the fitted mass. This procedure gives a $5 \mathrm{MeV} / c^{2}$ systematic uncertainty on the $W$ mass for the muon $p_{T}$ fit.

\subsection{Backgrounds}

Backgrounds to the measurement of the $W$ mass result from processes that mimic the decay signature of the $W$ boson. The sources of background in the muon channel are (from largest to smallest): $Z \rightarrow \mu \mu$ where one of the muons is lost, $W \rightarrow \tau \nu \rightarrow \mu \nu \nu \nu$, kaon decay, hadronic jets where one jet is mis-identified as a muon, and cosmic rays. These process have $p_{T}$ distributions that are significantly different from that of the $W$ decay. They generally have a lower mean $p_{T}$ and if not accounted for they will lower the fitted mass. We model each of these process in turn, and include them in our fitting templates. 


\subsection{1 $Z \rightarrow \mu \mu$ and $W \rightarrow \tau \nu$}

The $Z$ rejection requirement is very efficient in rejecting $Z$ events from the $W$ sample. However, due to incomplete muon detector coverage and inefficiencies, one muon from the $Z$ may go undetected and the event may be misidentified as a $W$ event. To model this background we generate $Z \rightarrow \mu \mu$ events with PYTHIA and run them through the fast detector simulation. We apply the $W$ selection criteria and measure the acceptance for $Z$ events. Then using the Standard Model ratio of $W$ to $Z$ cross-sections $\left(R=\sigma_{W} / \sigma_{Z}=\right.$ $10.67 \pm 0.45)$ we are able to estimate the total $Z$ background in our $W$ sample. We find a background of $(6.5 \pm 0.2) \%$.

We use an analogous procedure to calculate the uncertainty from the $W \rightarrow \tau \nu$ background. Events are generated with PYTHIA and run through the fast detector simulation. The acceptance is calculated. There is no additional correction factor for the relative Standard Model rates since the production cross-sections for $W \rightarrow \mu \nu$ and $W \rightarrow \tau \nu$ are equal. We find a background contribution from $W \rightarrow \tau \nu$ of $(0.9 \pm 0.1) \%$.

\subsubsection{Kaon Background}

What we call the "kaon background" actually refers to all long-lived particles that decay to a final state that includes a single muon. The dominant contribution to this is from $K \rightarrow \mu \nu$ decays. This background can be seen in the $t_{0}$ distribution of the sideband region $\left(0.2<\left|d_{0}\right|<0.6 \mathrm{~cm}\right)$ of the $W \rightarrow \mu \nu$ sample, where $t_{0}$ is the time at which particles cross the center of the COT chamber. There is a contribution to this from cosmic rays, however, this is flat for $t_{0}$ near zero, see Figure 5.2. The peak on top of the flat distribution of cosmics comes from kaon decays.

We estimate the kaon background from the track $\chi^{2}$ distribution of $W$ events. We find that a $(0.2 \pm 0.2) \%$ contribution from events obtained from the $d_{0}$ sideband sample gives the best fit between data and $\mathrm{MC}$. 


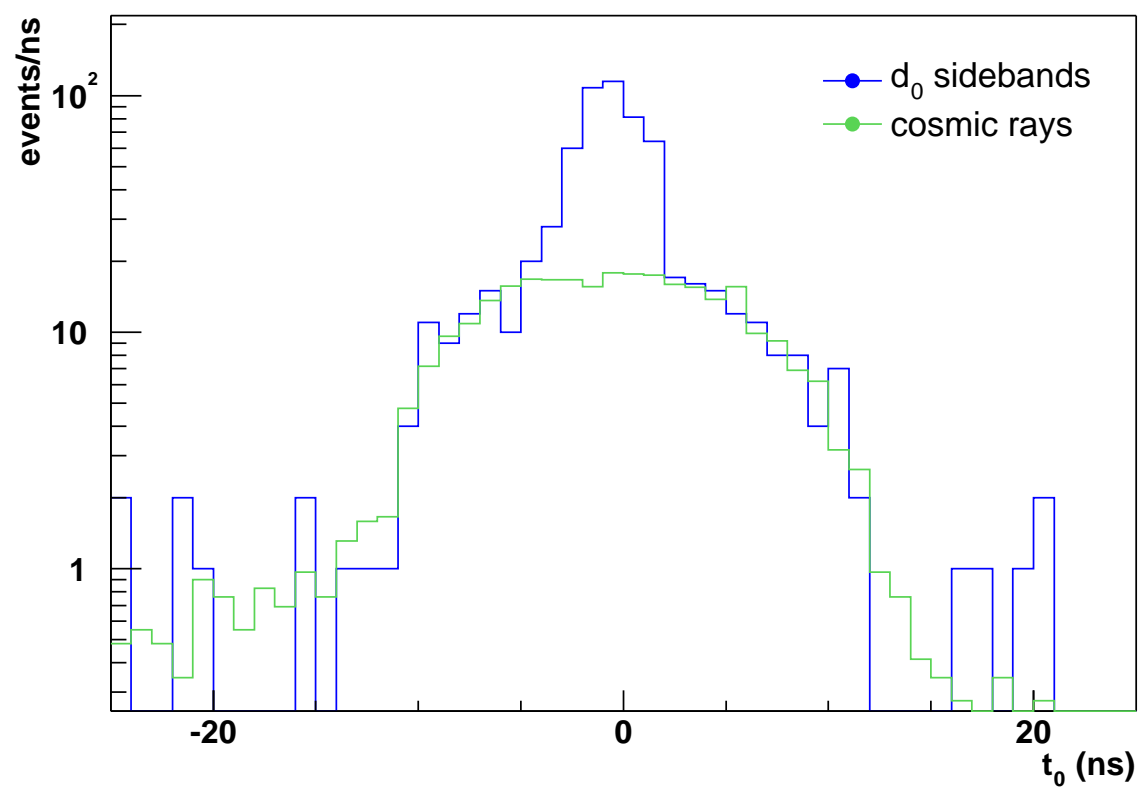

Figure 5.2: $t_{0}$ distributions of cosmics and $W \rightarrow \mu \nu$ in the sideband region. The peak near zero in the sideband sample comes from kaon decays.

We also check this background using the fact that most kaon events are strongly boosted so the muon candidate tends to be back-to-back with the hadronic recoil, $u$. In the plot of the azimuthal angle between the recoil and the muon, $\Delta \phi(u, l)$ (Figure 6.6), we observe a slight excess in the data when no background is included. The uncertainty is estimated by varying the background contribution and observing the change in the $\chi^{2}$ of the fit. We find a value consistent with zero.

Note that this background is greatly reduced by the cuts on the COT track of $\left|d_{0}\right|<$ $0.1 \mathrm{~cm}$ and $\chi^{2}<3$ and the cut on the hadronic recoil, $u<15 \mathrm{GeV}$. We find that with slightly looser cuts this background increases significantly. 


\subsubsection{Hadronic Jets}

Another source of background comes from hadronic jets where one jet is misidentified as a muon. We obtain QCD rich subsamples from the $W$ sample by selecting non-isolated events $(I s o>0.25)$ with inverted cuts on $E_{e m}, E_{h a d}$, and $\Delta x$ stub matching. We estimate the QCD background using several methods based on the fact that QCD events have low missing transverse energy and tend to be non-isolated.

The first QCD subsample we obtain is from the low $\mathbb{E}_{T}$ region. Using this sample we look at the calorimeter and track isolation (track isolation has an analogous definition to calorimeter isolation except instead of energy it uses the sum $p_{T}$ of the tracks). By adding QCD rich events to the $Z \rightarrow \mu \mu$ data (assumed to be free of QCD background) we obtain agreement with the $W$ data, see Figure 5.3.

The other QCD subsample we obtain is from the $I$ so $>0.3$ region of the $W$ sample. We correct this sample for contamination from the $W$ sample using Monte Carlo. Looking

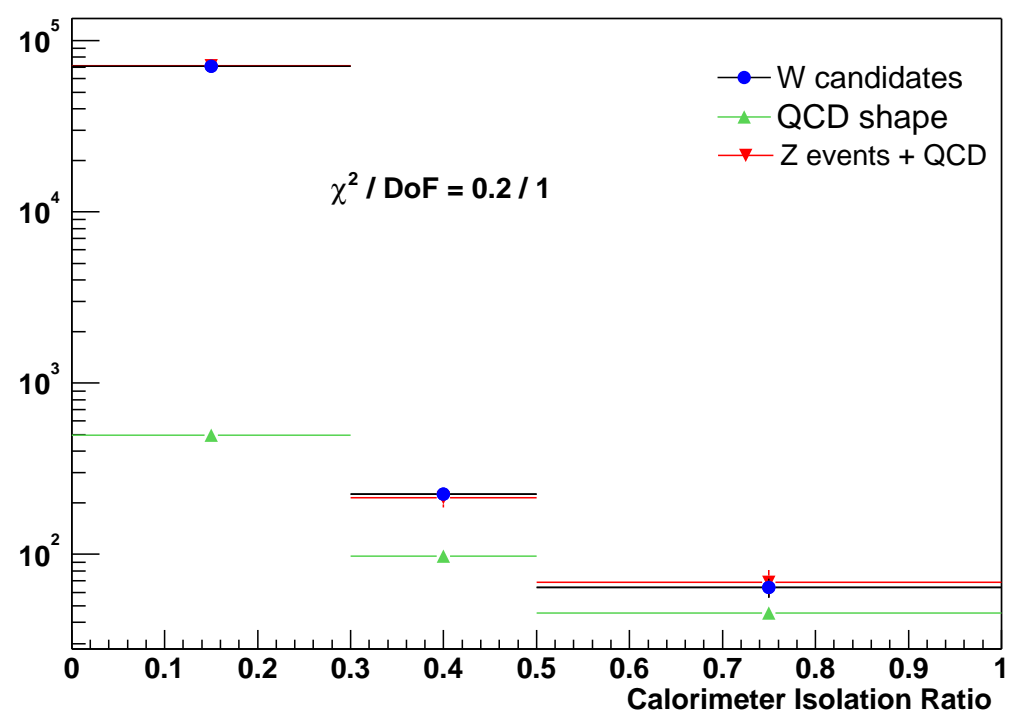

Figure 5.3: Calorimeter isolation for $W \rightarrow \mu \nu$ events and $Z \rightarrow \mu \mu$ with QCD added. The addition of QCD events to the $Z$ sample minimizes the $\chi^{2}$ of the fit to the $W$ data. 


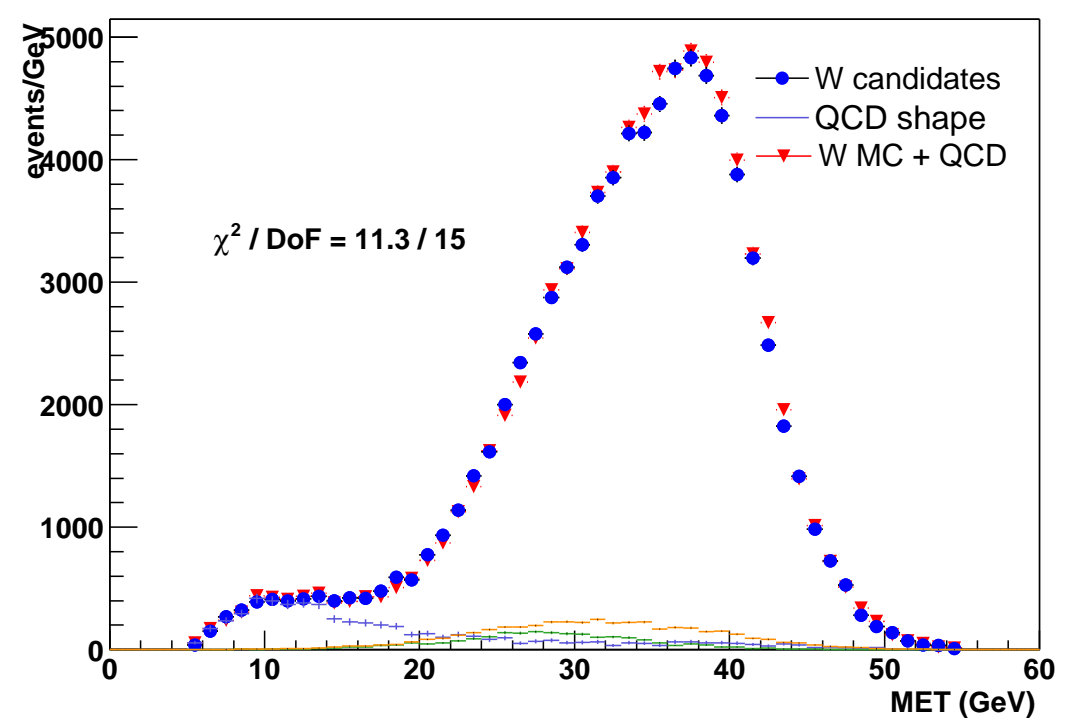

Figure 5.4: $\mathbb{E}_{T}$ distribution for $W \rightarrow \mu \nu$ data and $W$ Monte Carlo with QCD added. The addition of QCD events to the $W$ Monte Carlo minimizes the $\chi^{2}$ of the fit to the $W$ data.

at the $\mathscr{E}_{T}$ distribution of the $W$ sample we see a collection of events at low $\mathscr{H}_{T}$ due mostly to QCD background, see Figure 5.4.

We find the combination of the above methods gives a QCD background value consistent with zero, namely, $(0.05 \pm 0.05) \%$.

\subsubsection{Cosmic Background}

A small background contribution to the $W$ sample can come from high energy muons in cosmic rays. The majority of cosmic ray events are removed by an algorithm (called a cosmic tagger) [37] that looks for a track opposite the identified muon and performs a single fit to these two tracks. The remaining background is modelled using the $t_{0}$ distribution. Since cosmics are continuously arriving in the detector volume the $t_{0}$ distribution should be reasonably flat. Whereas we would expect particles produced in the $p \bar{p}$ collision to have $t_{0}$ values that coincide with the beam-crossing time $\left(t_{0}=0\right)$. This is apparent 
in the $t_{0}$ distribution shown in Figure 5.5. We can see $W$ and $Z$ events peak at zero, whereas the cosmic ray events are roughly flat over the small $t_{0}$ regime. We estimate the cosmic background in the $W$ sample by normalizing the $t_{0}$ sideband region of the $W$ events to that of the cosmic events. We do this for several different sideband regions between 7 and 25 ns.

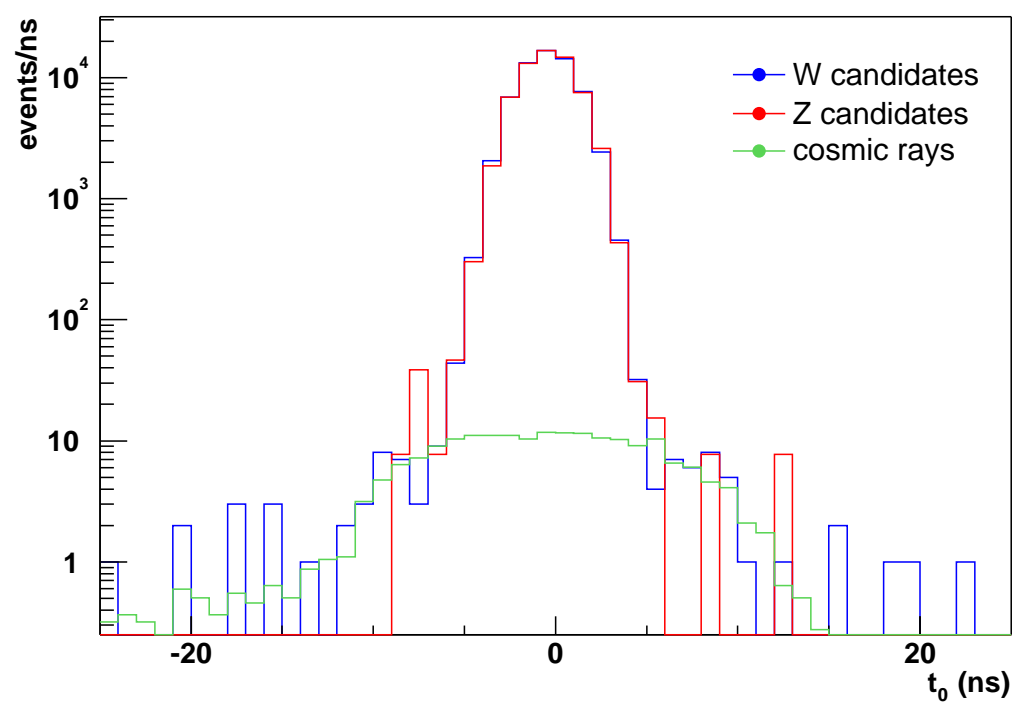

Figure 5.5: $t_{0}$ distributions of $W$ data, $Z$ data, and cosmic ray muons. We can see the $Z$ sample is essentially free of cosmic background events. We normalize the $W$ sample to the cosmics in the $t_{0}$ sideband region to estimate the cosmic background.

We obtain a second estimate of the cosmic background from the $d_{0}$ sideband region, see Figure 5.2. Other than the peak from kaon decays, the $d_{0}$ sidebands of the $W$ sample are taken to be entirely comprised of cosmic ray events. These two methods combined yield a final background estimate of consistent with zero, $(0.05 \pm 0.05) \%$. 


\subsubsection{Combined Background}

In addition to the overall normalizations of the backgrounds obtained above, we obtain the $p_{T}$ spectrum of each background from samples that are entirely composed of background events. The $Z \rightarrow \mu \mu$ and $W \rightarrow \tau \nu$ shapes are obtained from Monte Carlo, the kaons from the $d_{0}$ sidebands of $W$ data, and the cosmics from the $W$ data with the cosmic tagger inverted. The QCD shape is obtained from the QCD subsample described in section 5.2.3. The $p_{T}$ spectrum of all the backgrounds is shown in Figure 5.6. We vary the shapes of each background to gauge the associated systematic uncertainty on the $W$ mass.

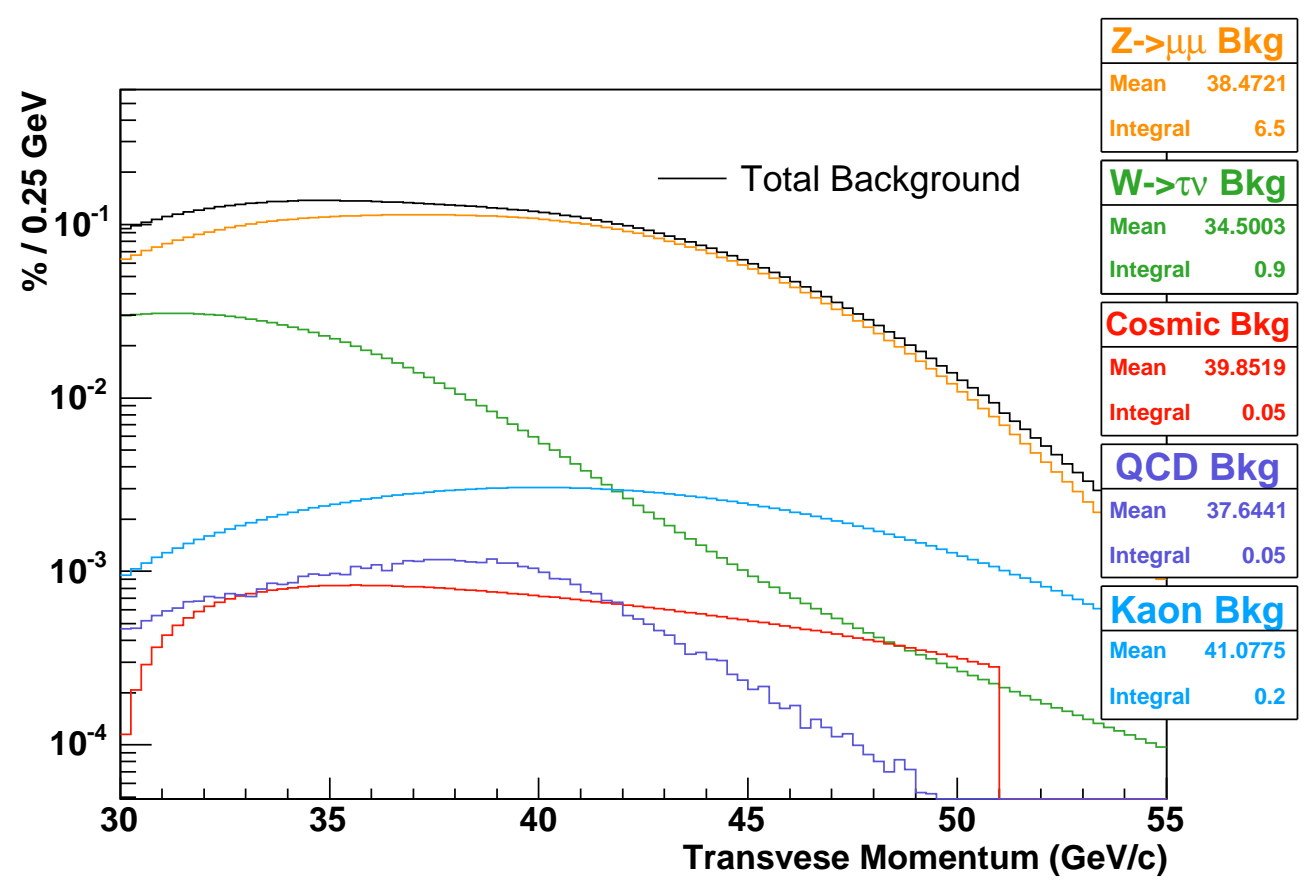

Figure 5.6: The $p_{T}$ spectra of all the backgrounds to $W \rightarrow \mu \nu$. We can see most of the backgrounds peak at lower $p_{T}$ values than the $W$ signal.

These backgrounds are included in the final fitting templates. We estimate the systematic uncertainty on the $W$ mass arising from both the normalization and shape of the backgrounds to be $20 \mathrm{MeV} / \mathrm{c}^{2}$. 


\section{Chapter 6}

\section{Fast Detector Simulation}

The particles produced by the event generation process (charged leptons, neutrinos, and photons) are individually run through a so-called fast detector simulation [38] that simulates their interaction with the CDF detector. We do not directly use the standard $\mathrm{CDF}$ detector simulation (cdfSim) since it is prohibitively slow ( $\sim 1$ event/s) for the large sample sizes we work with (e.g. tens of millions of events) and doesn't have tunable parameters that can be used to investigate various systematic effects.

The fast simulation models the COT response and resolution, muon acceptance and efficiency, and the calorimeter response and resolution to the hadronic recoil. In addition, all material inside the COT is accounted for by way of a material map, generated from cdfSim [39]. The fast simulation parametrizes the response and resolution of the detector by way of a number of tunable parameters set by various quantities measured from data.

The fast simulation reads in files containing kinematic information of particle decays and generates templates that are then used by a fitter (see section 8.1) to find the best fit mass. For $W$ bosons the fast simulation processes one file generated with $M_{W}=80$ $\mathrm{GeV} / c^{2}$ and another with $M_{W}=81 \mathrm{GeV} / c^{2} \mathrm{GeV}$. Each of these mass points are fully simulated at the rate of approximately 100 events/s. Then a linear extrapolation in 1.25 MeV $/ c^{2}$ steps is performed between these two fully simulated templates producing 
a series of 800 templates suitable for fitting to the data. An analogous procedure is used for $Z$ boson templates. For $J / \psi$ and $\Upsilon$ events, the fast simulation produces a series of templates by varying the momentum scale about unity. The fast simulation allows us to investigate numerous systematic uncertainties by varying parameters and observing the shift in the measured $W$ mass.

\subsection{Material Modelling}

Precise knowledge of the amount of material inside the detector is crucial to the $W$ mass measurement, since this information is needed to calculate the energy lost by the charged lepton, to evaluate the probability of radiating a bremsstrahlung photon, and to evaluate the probability of a photon undergoing the conversion process, $\gamma \rightarrow e e$. For the muon channel measurement, the most important of these processes is ionization energy loss. We use a material map generated from scanning the full GEANT 4 [40] cdfSim detector geometry. Three material properties are measured: the number of radiation lengths, $X_{0}$, needed for evaluating bremsstrahlung and conversion probabilities, and the normalization and ionization constants need for evaluating the Bethe-Bloch formula [7] for ionization energy loss. These properties are stored in a three dimensional look-up table as a function of $r, z$, and $\phi$. We validate the material map using a large sample of cosmic ray muons, since the decay muons are sensitive to the amount of material via ionization energy loss. Final tuning of the ionizing material in the detector is accomplished by studying the dependence of $J / \psi \rightarrow \mu \mu$ invariant mass on the mean curvature, described in section 7.1. The number of radiation lengths is tuned using the tail of the $E / p$ distribution from $W \rightarrow e \nu$, which is sensitive to bremsstrahlung.

\subsection{COT Simulation}

The kinematic information provided by the generator level file is used to propagate the particles along a helical trajectory outward from the production point. The fast simula- 
tion provides the option to beam-constrain the track. Doing so significantly improves the momentum resolution since the beamspot provides an extra point in the track fit at least $40 \mathrm{~cm}$ from the nearest COT hits. The beamspot has been measured from reconstructed event vertices in data [41] and is approximately $30 \mu \mathrm{m}$ in $x$ and $y$ with an uncertainty of about $5 \mu \mathrm{m}$. We find a beamspot size of $39 \pm 3 \mu \mathrm{m}$ in $x$ and $y$ gives the best agreement between data and $\mathrm{MC}$ in the $Z \rightarrow \mu \mu$ invariant mass fit, consistent with measured values. At each layer of passive material, as represented by the material map, the stored material properties are used to evaluate the ionization energy loss of the charged lepton as determined by the Bethe-Bloch formula. In addition, the charged lepton is deflected by the predicted amount of multiple Coulomb scattering at each layer. We use the following equation to model multiple scattering [7],

$$
\theta_{0}=\frac{13.6 \mathrm{MeV}}{\beta c p} z \sqrt{x / X_{0}}
$$

where $p$ is the particle momentum in $\mathrm{MeV}, \beta c$ is the velocity, $z$ is the charge, $\sqrt{x / X_{0}}$ is the material thickness in radiation lengths. $\theta_{0}$ is the width of a Gaussian distribution that describes the small angle scattering of the particle. In addition to this Gaussian component, the multiple scattering distribution has a non-Gaussian component from large angle scatters. To account for both components, we use a $98 \%$ Gaussian "core" distribution with a $2 \%$ non-Gaussian tails. To estimate the width of the non-Gaussian component, we fit data from the MUSCAT collaboration [42] with two Gaussians, normalized to $98 \%$ and $2 \%$ of the total, see Figure 6.1 . We find the wide angle scattering can be adequately modelled with a Gaussian that is 3.8 times wider than the core Gaussian.

Simulated hits in the COT are digitized and smeared according to a Gaussian distribution with a resolution determined by $J / \psi, \Upsilon$, or $Z$ decays to muons. A helix is fit to the hits to determine the helix parameters: $d_{0}, \phi_{0}, z_{0}, c, \cot \theta$, and $t_{0}$, which are, the distance of closest approach of the fitted track to the origin, the azimuthal angle at $d_{0}$, 


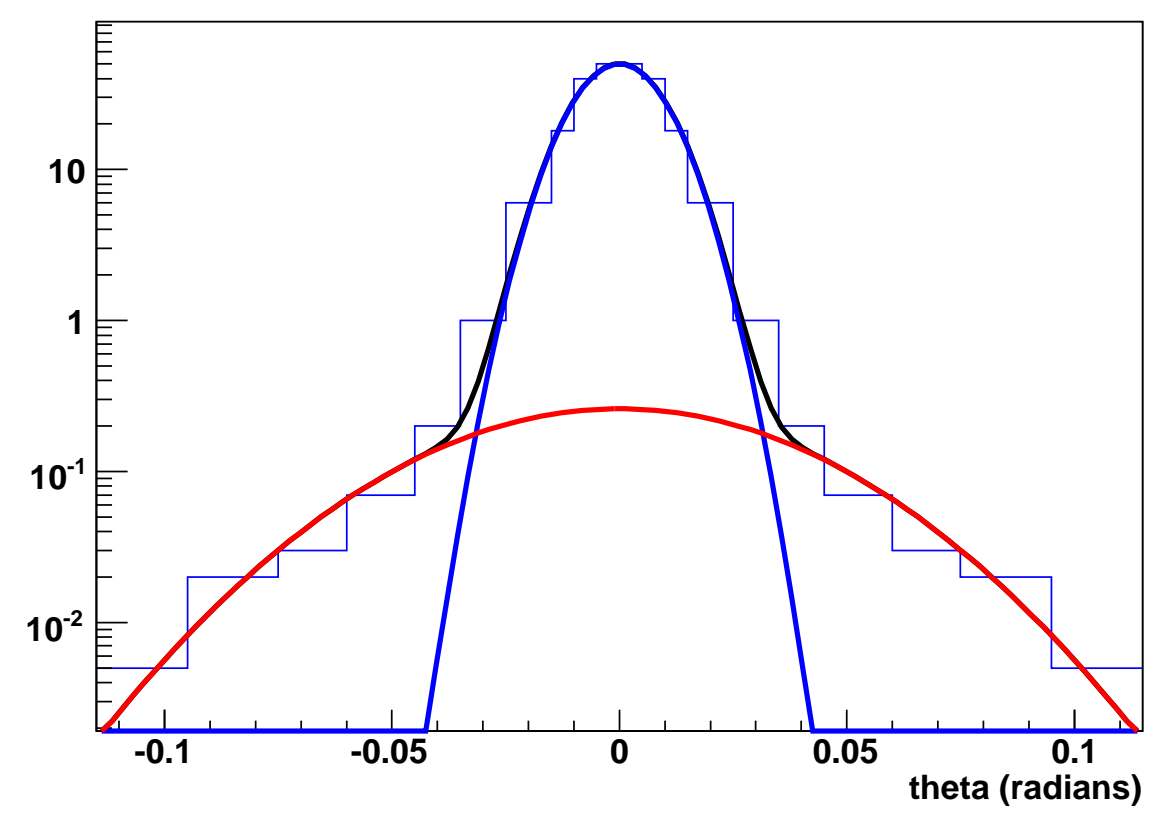

Figure 6.1: Double Gaussian multiple scattering model fit to MUSCAT data. The narrow Gaussian "core" shown in blue is normalized to $98 \%$ of the total. The wide Gaussian shown in red represents the remaining $2 \%$. The two Gaussians combined fit the data (blue histogram) well.

the $z$ coordinate at $d_{0}$, the curvature of the track (inverse diameter in $r-\phi$ space), the dip angle, and the time at which the track crosses the center of the COT, respectively. The transverse momentum is obtained from the formula, $p_{T}=0.3 B r=0.00211593 /|c|$, where $B$ is the magnetic field in Tesla, $r$ is the radius of curvature in centimeters, and $|c|$ is the magnitude of the curvature. Both $d_{0}$ and $\phi_{0}$ are updated at each layer of passive material as a result of the amount of energy loss.

Alignment of the COT is performed using a large sample of cosmic rays [43]. This procedure involves fitting a single helix to the incoming cosmic track and the outgoing cosmic track, and computing the residuals for each. An additional correction to the curvature of the track is needed to correct for wire shifts from their nominal positions. 
This alignment is performed by measuring the difference in $E / p$ for positrons and electrons from $W \rightarrow e \nu$ decay. We find the above correction shifts the $p_{T}$ fit $W$ mass by 10 $\mathrm{MeV} / c^{2}$. We take this as a systematic uncertainty on the final measurement.

\subsection{Muon Acceptance Modelling}

The acceptance of the detector for muons is modelled from cdfSim coupled with a cut on $z$ of the muon of $|z|<155 \mathrm{~cm}$, i.e. the distance of the COT endplates from the center of the detector. The $z$ cut requires the muon to be within the fiducial volume of the COT. Single Monte Carlo muons are fired through cdfSim and the probability of identifying the reconstructed muon in the CMUP or CMX is computed as a function of $\phi$ and $\cot \theta$ ( $z$ dependence is negligible). Since $\phi$ and $\cot \theta$ show little correlation, we store the efficiencies $\epsilon_{\phi}$ and $\epsilon_{\cot \theta}$ in histograms for CMUP and CMX separately. To maximize these efficiencies we scale the CMX histograms so that the maximum bin is unity. In order to preserve the ratio between CMUP and CMX observed in the data we then scale the CMUP histograms so that the maximum efficiency is 0.86 . The complete efficiency for identifying a muon is,

$$
\epsilon^{\mu}(\phi, \cot \theta)=\epsilon_{\phi}^{\mathrm{CMUP}}(\phi) \cdot \epsilon_{\cot \theta}^{\mathrm{CMUP}}(\cot \theta)+\epsilon_{\phi}^{\mathrm{CMX}}(\phi) \cdot \epsilon_{\cot \theta}^{\mathrm{CMX}}(\cot \theta)
$$

\subsection{Hadronic Recoil}

An important aspect of the $W$ mass measurement is accurately modelling the calorimeter response and resolution to the particles recoiling against the $W$ boson, referred to as the hadronic recoil $(u)$. The modelling of the recoil influences the $p_{T}$ muon mass measurement through kinematic dependencies, such as the cut on $u$ of $15 \mathrm{GeV}$. In addition, the recoil is used as a check of the kaon background. The hadronic recoil is measured as the sum of 
all calorimeter towers, excluding the towers which contain the energy cluster of the muon (minimum ionizing energy). Defining it in this way means that $u$ not only contains the component coming from the hadrons recoiling against the boson but also a component from the underlying event. The underlying event arises from two distinct processes: proton/antiproton remnants from the interaction that produced the $W$ boson, referred to as spectator interactions, and additional interactions in the same beam-crossing, referred to as multiple interactions.

It is convenient to decompose $u$ into components parallel $\left(u_{\|}\right)$and perpendicular $\left(u_{\perp}\right)$ to the direction of the muon, see Figure 6.2. This gives the magnitude of $u_{\|}$as the average calorimeter response to hadrons recoiling against the $W$; this directly influences $M_{T}^{W}$ and even more so $\mathbb{E}_{T}$. Any biases in $u_{\|}$translate directly into biases on the measured $W$ mass from these fit variables. The muon $p_{T}$ fit is not influenced by $u_{\|}$at first order since unlike the previous quantities, the calculation of $p_{T}$ doesn't make use of the measured recoil. The perpendicular component of $u$ should have an average value of zero, since its measurement only depends on the calorimeter resolution to $u$.

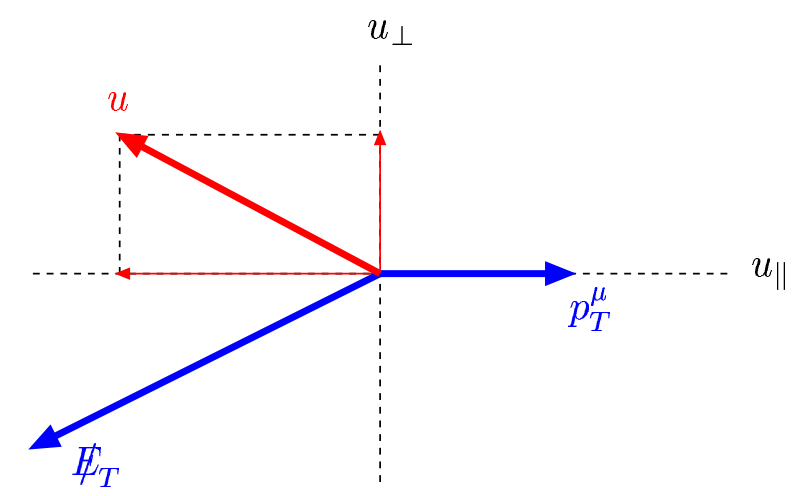

Figure 6.2: Schematic diagram showing $u_{\|}$and $u_{\perp}$ directions. The $u_{\|}$direction is defined as the direction parallel to the muon $p_{T}$. This particular diagram shows an event with a negative $u_{\|}$and a positive $u_{\perp}$. 


\subsubsection{Muon Removal Correction}

As mentioned above, when computing the hadronic recoil we need to account for the underlying event energy in the calorimeter towers containing the minimum ionizing energy left by the muon. Figure 6.3 shows the mean EM and hadronic $E_{T}$ per tower surrounding the muon track in $W \rightarrow \mu \nu$ data.
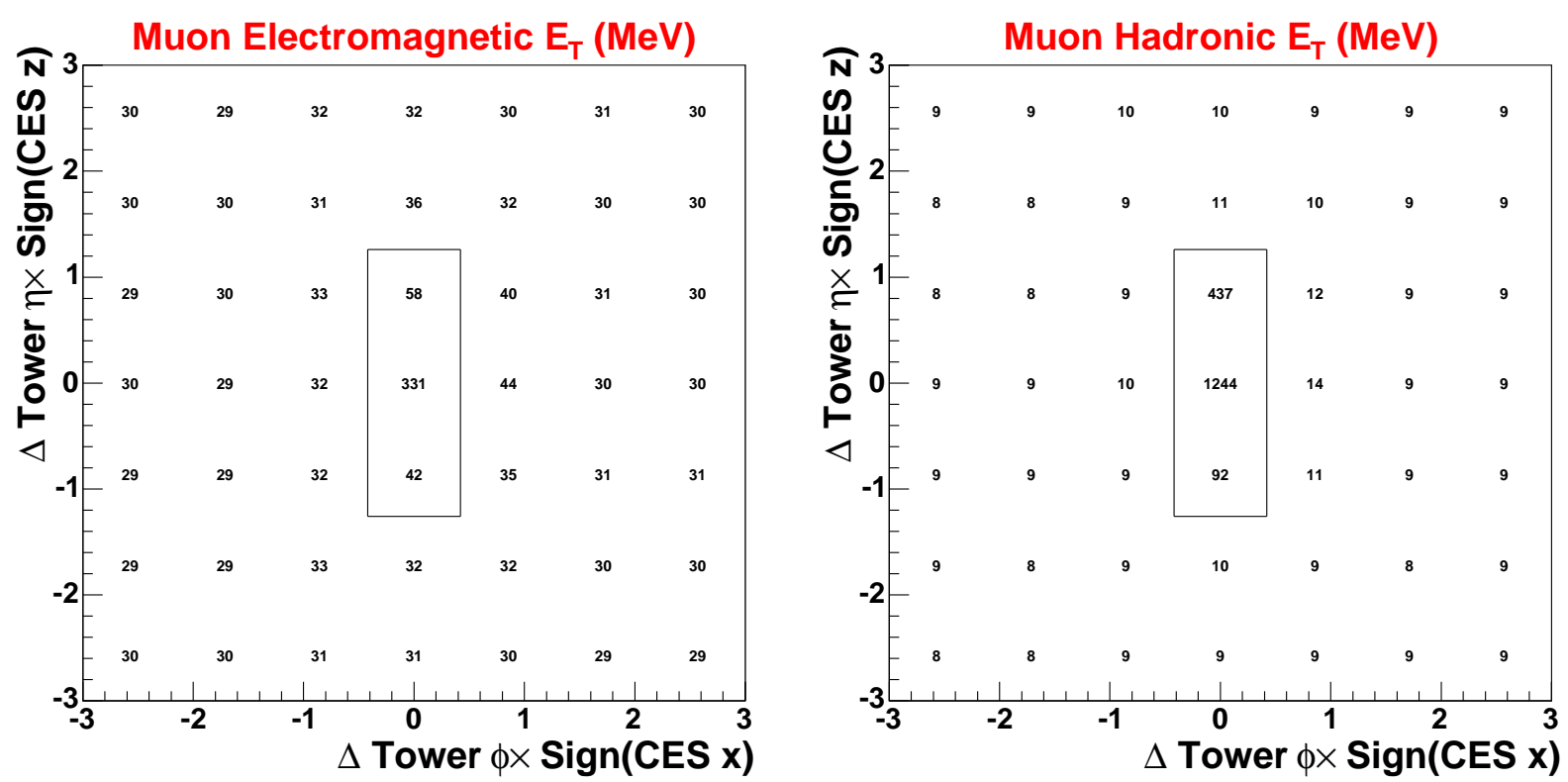

Figure 6.3: Mean EM and hadronic energy in each tower surrounding the muon track. Using signed CES $z$ in the $\eta$ direction means that the tower closest to the primary muon tower is always in the positive $\eta$ direction, causing an apparent bias in tower energies.

Based on this we exclude three towers in the computation of $u$ as indicated on the plot. Excluding these towers not only removes the energy deposited from the muon, but also removes the underlying event energy in these towers. We estimate how much underlying event energy is removed by measuring the energy in towers separated in $\phi$ from the muon. This energy shows some dependence on $\eta, u_{\|}$, and $u_{\perp}$. These dependencies are incorporated into the fast simulation by scaling factors and the mean energy is then subtracted off the recoil in the simulation. 


\subsubsection{Determining the Recoil Model Parameters}

The response and resolution of the calorimeter to the hadronic recoil is parametrized using a model with parameters set using $Z$ decays to muons and electrons. We can accurately measure in data the $p_{T}$ of the two charged leptons from the $Z$ decay, and hence construct the $p_{T}$ of the $Z$ boson itself. We can then compare the "true" $Z$ boson $p_{T}$ with what we obtain from the measurement of the hadronic recoil. It is useful to define a coordinate system with one axis as the $p_{T}$ weighted bisector of the charged lepton pair $(\eta$-axis $)$ and the other axis perpendicular to that ( $\xi$-axis), see Figure 6.4.

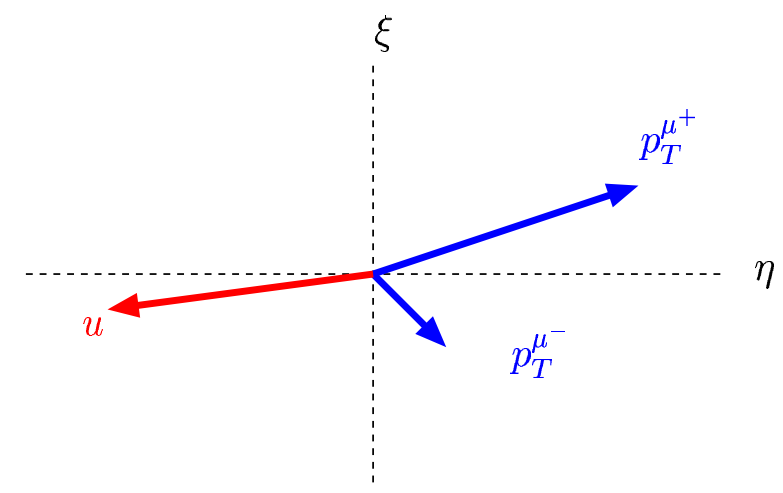

Figure 6.4: Definition of the $\eta-\xi$ coordinate system used for the recoil model. The $\eta$ axis is defined as the $p_{T}$ weighted bisector of the di-lepton $p_{T}$ vectors.

For the calorimeter scale, we define a response function $R$ given by,

$$
\begin{aligned}
R & \equiv u_{\text {rec }} / u_{\text {true }} \\
& =a \frac{\log \left(u_{\text {true }}+b\right)}{\log (15+b)}
\end{aligned}
$$

where $u_{r e c}$ is the reconstructed recoil, $u_{t r u e}$ is the "true recoil", i.e. the negative of the $p_{T}^{W}$ vector as given by the Monte Carlo, and $a$ and $b$ are positive constants. This functional form was chosen to decouple the parameters $a$ and $b$. We plot the $p_{T}$-balance $\left(p_{T}+u\right)$ in both the $\eta$ and $\xi$ directions. The parameters $a$ and $b$ are determined from the best fit to 
the $p_{T}^{\eta}$-balance plot, see Figure 6.5. We find values of $a=0.700 \pm 0.007$ and $b=19.8 \pm 4.4$ $\mathrm{GeV}$.
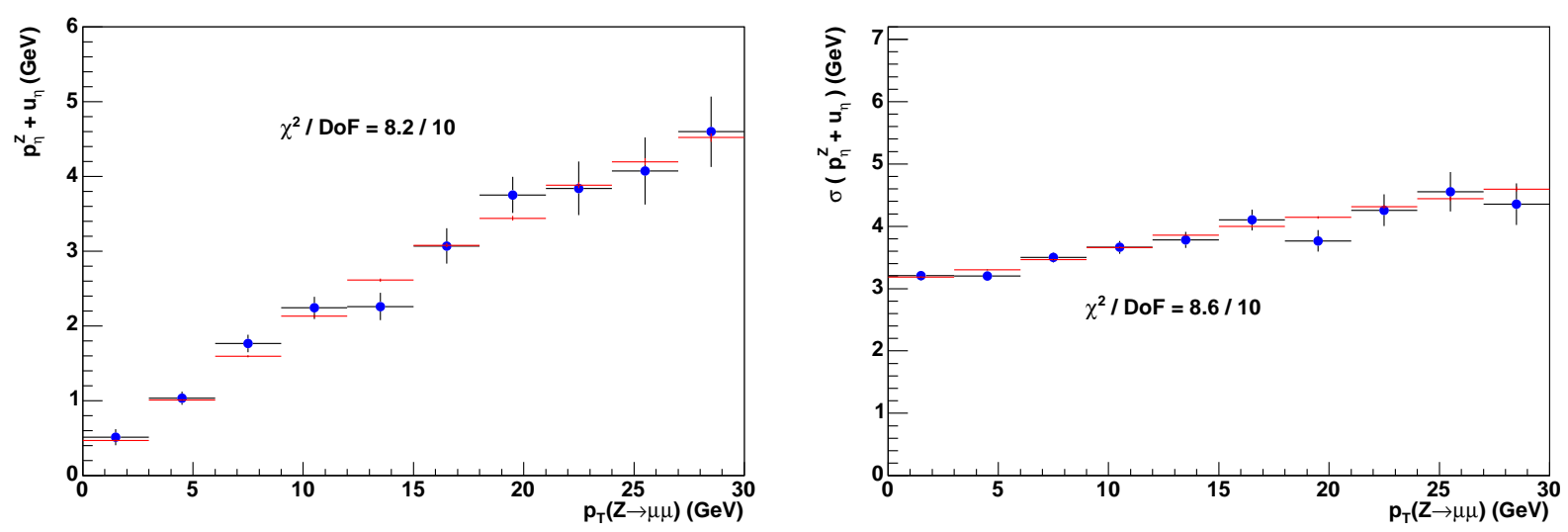

Figure 6.5: Mean and RMS $p_{T}^{\eta}$-balance plots for $Z \rightarrow \mu \mu$. The plot of the mean $p_{T^{-}}^{\eta}$ balance is used to determine the recoil scale. The plot of $\sigma\left(p_{T}^{\eta}+u^{\eta}\right)$ is used to tune the resolution model. Note that analogous plots for $Z \rightarrow e e$ are also used to tune the model $[44]$.

To model the recoil resolution we use two parameters, one for the high $p_{T}$ regime (the calorimeter "jet" resolution) and one for low $p_{T}$ (the underlying event resolution). The jet resolution is given by a hadronic sampling term, $s_{\text {had }} \sqrt{u_{\text {true }}}$. We find from the plot of $\sigma\left(p_{T}^{\eta}+u^{\eta}\right)$ a value of $s_{\text {had }}$ of 0.12 . To model the underlying event resolution we parametrize the $\Sigma E_{T}$ distribution from minimum bias data. We sample this distribution and apply a scale factor to the $\Sigma E_{T}$. We find a scale factor of $N=1.36 \pm 0.03$ gives the best agreement between data and Monte Carlo in the $\sigma\left(p_{T}^{\eta}+u^{\eta}\right)$ plot. We use the same value for both $W$ and $Z$ events. The underlying event is parametrized with a random two-dimensional vector that is a function of $\Sigma E_{T}$. We then apply the model to $W \rightarrow \mu \nu$ events. Plots of $u, u_{\|}, u_{\perp}$, and $\Delta \phi(u, \mu)$ between data and $\mathrm{MC}$ are shown in Figure 6.6.

In summary, the recoil model consists of a calorimeter response function $R$, that contains two parameters, $a$ and $b$. Varying these parameters by their respective uncertainties yield systematic uncertainties on the $W$ mass of $5 \mathrm{MeV} / c^{2}$. The other aspect of the model 
is the calorimeter resolution to the recoil, which is governed by the parameters $s_{h a d}$ and $N$. Varying these parameters contributes a $7 \mathrm{MeV} / c^{2}$ systematic uncertainty to the $W$ mass. 

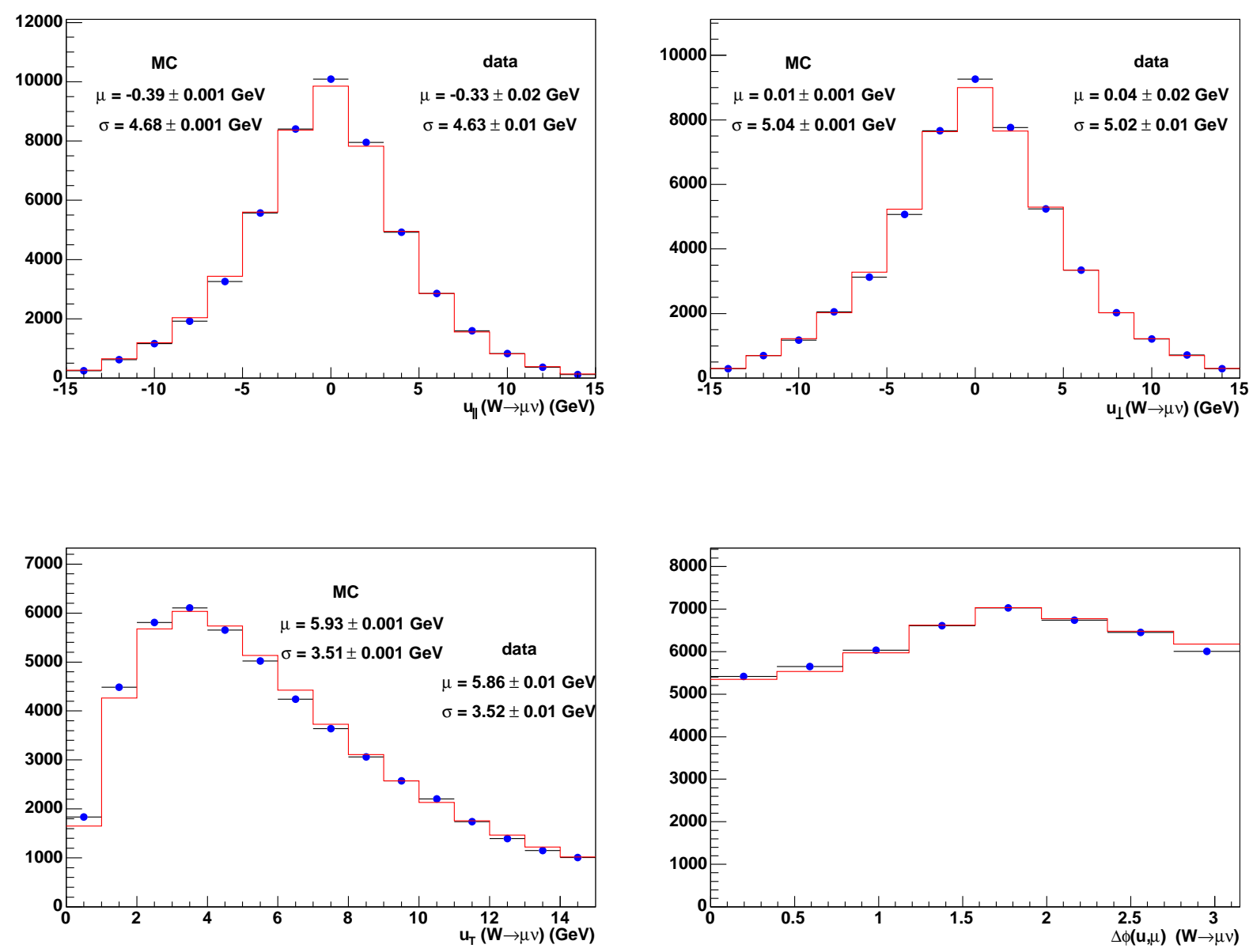

Figure 6.6: Recoil model comparison plots for $W \rightarrow \mu \nu$. The recoil model derived from $Z$ events and minimum bias data is applied to the $W \rightarrow \mu \nu$ data. Data and MC show good agreement. Note that backgrounds are included in these plots. The $\Delta \phi(u, \mu)$ distribution is used to check the kaon background (section 5.2.2). 


\section{Chapter 7}

\section{Detector Calibration}

A major aspect of the $W$ mass analysis is calibrating the detector. The relevant calibration for a muon channel measurement is determining the momentum scale of the COT tracker. This amounts to a calibration of the magnetic field. The momentum scale is a correction factor that is applied to the momenta of all COT tracks. By measuring the invariant mass of the well known $J / \psi \rightarrow \mu \mu$ and $\Upsilon(1 S) \rightarrow \mu \mu$ resonances, we are able to extract the momentum scale from a fit based on how far the measured masses are from their world average values. We could also determine the momentum scale from $Z \rightarrow \mu \mu$ events. In fact, since the mean $p_{T}$ of $Z$ bosons is close to the mean $p_{T}$ of $W$ bosons, this would appear to be the best method. However, the $J / \psi$ and $\Upsilon$ samples have the advantage of much higher statistics. Furthermore, these samples allow us to investigate various detector effects. We use the $\Upsilon$ sample to study the effect of beam-constrained vs. non beam-constrained tracks. The high statistics of the $J / \psi$ sample allows us to tune the amount of ionizing material in the detector simulation. We take the weighted average of the $J / \psi$ and $\Upsilon(1 S)$ scales as the final momentum scale. We cross-check this result by measuring the $Z \rightarrow \mu \mu$ invariant mass with the scale applied. 


\subsection{COT Momentum Scale from $J / \psi \rightarrow \mu \mu$}

The main benefit of using this sample is that it is large enough that we can study the dependence of the momentum scale on numerous variables, such as angular separation of the two muons and the curvature of the track. One drawback is that the $J / \psi$ invariant mass $\left(3096.92 \pm 0.01 \mathrm{MeV} / c^{2}[7]\right)$ is much smaller than the $W$ invariant mass, making for a large extrapolation to this higher mass scale. This means any systematic uncertainties on the momentum scale from $J / \psi \rightarrow \mu \mu$ will be inflated upon extrapolation to the $W$ mass.

\subsubsection{Fitting templates}

We use PYTHIA to generate approximately $60 \times 10^{6} \mathrm{~J} / \psi \rightarrow \mu \mu$ events. We observe that there are initially some kinematic differences between the Monte Carlo and the data. In particular, the $p_{T}$ distribution of the muon pair and sum of the two muon curvatures do not match well between data and Monte Carlo.

To correct for this, we tune the output of PYTHIA by increasing the rapidity of the decay muons in the direction of $p_{T}^{\mu \mu}$, effectively increasing the di-muon $p_{T}$. We also tune the polar angle of the muon (in the di-muon rest frame) such that the sum curvature distributions agree. Figure 7.1 shows the agreement between data and Monte Carlo after tuning.

Photons radiated from the muons can have a significant impact on the measured momentum scale. Since PYTHIA does not account for this, we include QED radiation in the fast simulation by way of a form factor [36],

$$
f_{\mu}^{\mu}(x)=\beta(1-x)^{\beta-1}
$$

where $f_{\mu}^{\mu}(x)$ is the fragmentation probability of the muon, $x$ is the energy fraction retained by the muon, and $\beta$ is a constant given by 

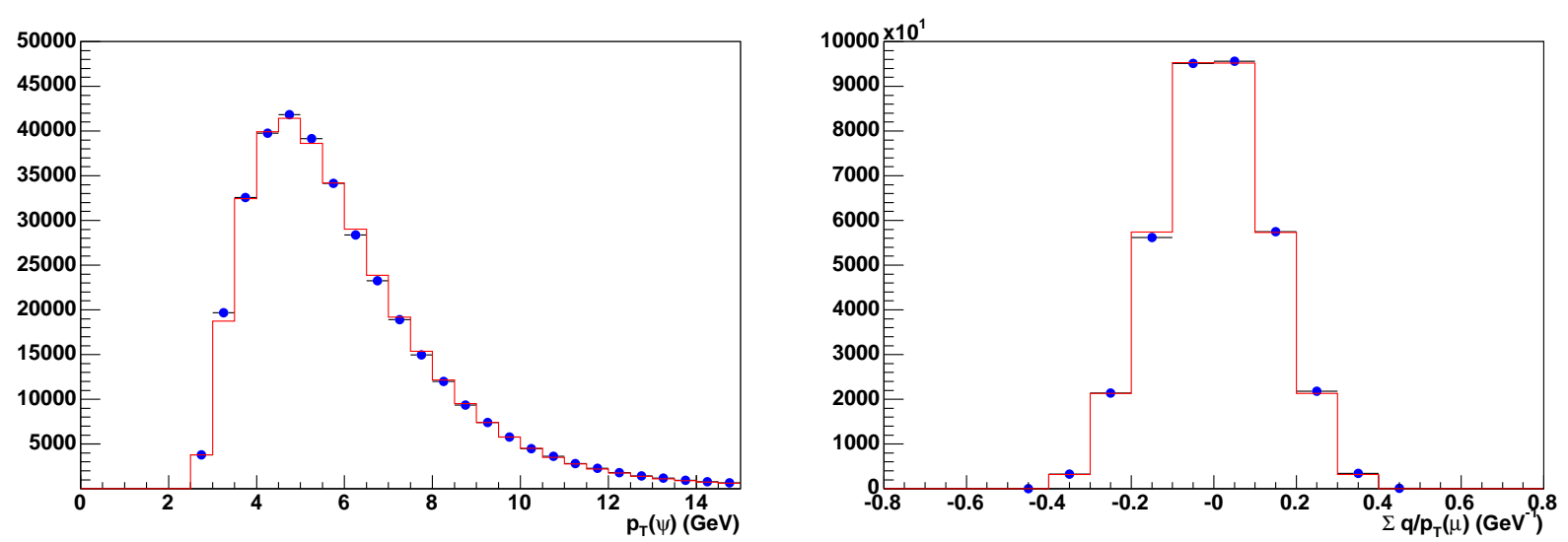

Figure 7.1: Di-muon $p_{T}$ and sum curvature distributions after tuning PYTHIA.

$$
\beta=\frac{\alpha}{\pi}\left(2 \ln \frac{Q}{m_{\mu}}-1\right)
$$

where $Q$ is the energy scale at which the fragmentation takes place $\left(M_{J / \psi}\right.$ in this case) and $\alpha$ is the fine-structure constant.

Templates are generated in the fast simulation as a function of the difference of the momentum scale from unity.

\subsection{2 $J / \psi$ Analysis}

Backgrounds to $J / \psi \rightarrow \mu \mu$ are accounted for by way of a method called sideband subtraction. We define the region $3.01<M_{J / \psi}<3.15 \mathrm{GeV} / c^{2}$ as the signal region and take the region $3.17<M_{J / \psi}<3.31 \mathrm{GeV} / c^{2}$ as the "sideband" region, in which it is assumed there is negligible signal, see Figure 7.2. Note that we take the sideband region from above the $J / \psi$ peak since the low side is sensitive to QED radiation. We observe a flat distribution in the sideband region, which we then subtract off the signal.

The high statistics of the $J / \psi$ sample exposes some detector effects that cannot be seen with smaller samples such as $\Upsilon \rightarrow \mu \mu$. One such effect is a relative rotation of east 


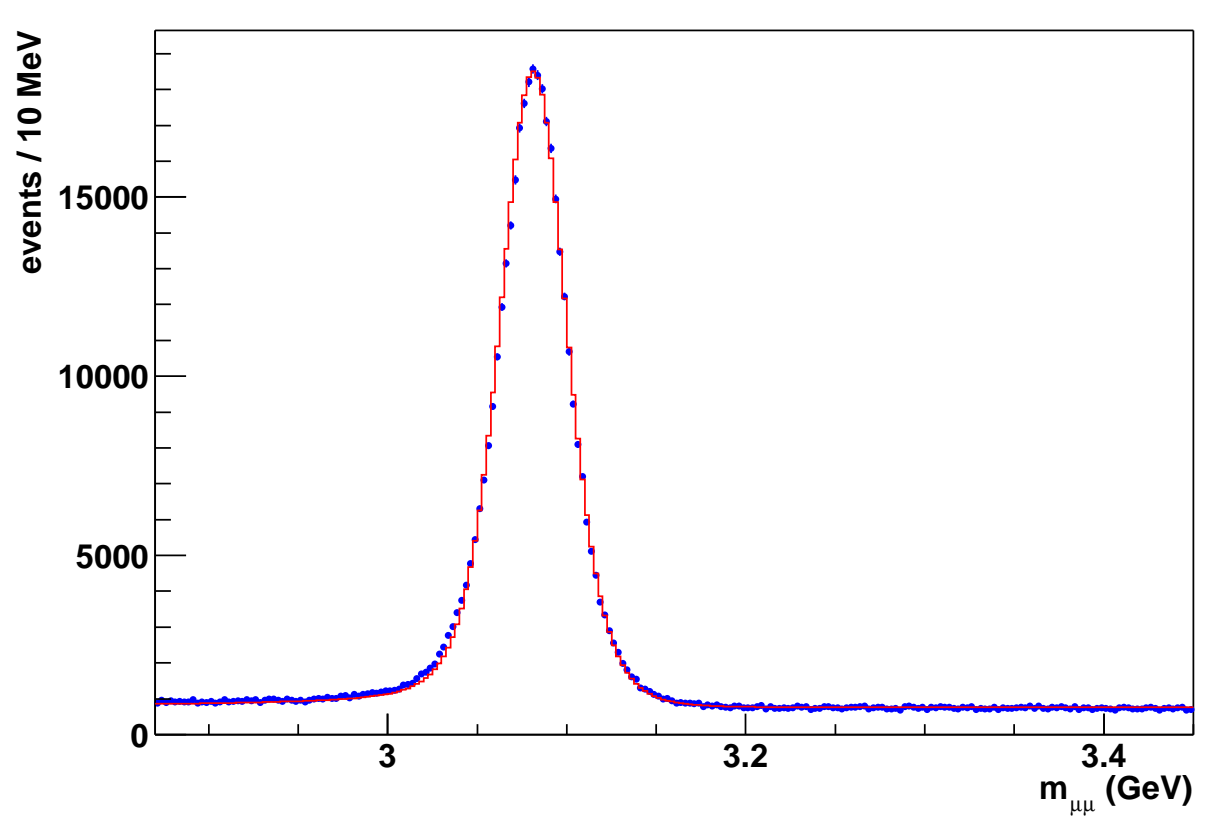

Figure 7.2: Invariant mass of $J / \psi \rightarrow \mu \mu$. This distribution is used for several purposes. We extract the background to $J / \psi \rightarrow \mu \mu$ using sideband subtraction via this plot. We estimate the QED systematic by varying the $Q$ scale to minimize the $\chi^{2}$ of the fit between data and Monte Carlo. The width of this distribution is used to determine the COT single hit resolution.

and west COT endplates. Another effect is small deviations of the stereo angles of COT wires from their nominal positions.

The dependence caused by differing wire positions can be removed by applying a scale factor to the measured $\cot \theta$ of the track,

$$
\cot \theta \rightarrow 0.9997 \cdot \cot \theta
$$

The dependence caused by endplate rotation can be removed by making a correction to the measured curvature, 


$$
c \rightarrow c-7.0 \times 10^{-7} \cdot \cot \theta
$$

Both these corrections have an uncertainty of 1 in the last digit. Figure 7.3 shows the scale as a function of $\Delta \cot \theta$ after the above corrections were applied. The corrections were obtained by requiring the plot to be flat.

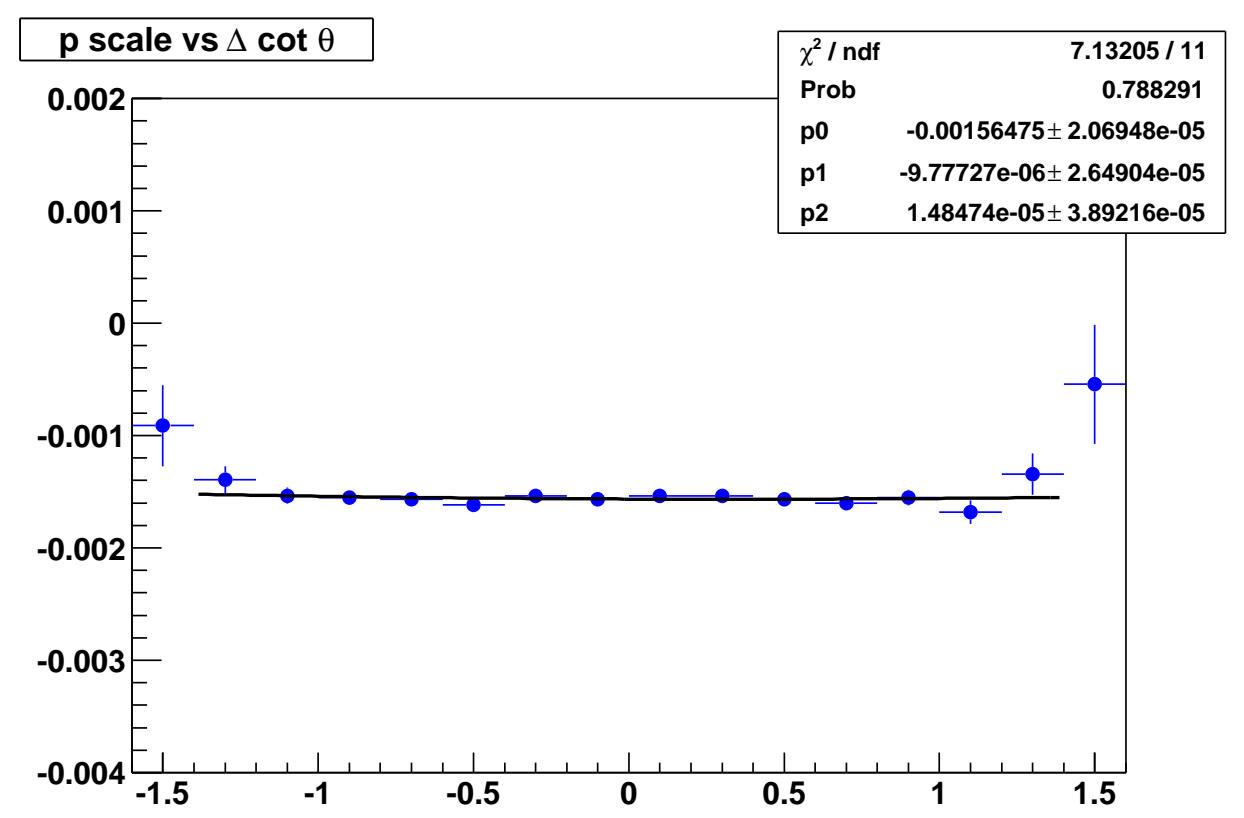

Figure 7.3: $\Delta p / p$ vs. $\Delta \cot \theta$ between the $\mu^{+}$and $\mu^{-}$in $J / \psi$ decay. The corrections given in equations 7.3 and 7.4 have been applied to make this plot flat.

Another variable that the momentum scale is sensitive to is the amount of ionizing material in the detector, as this governs the dominant energy loss mechanism for muons. When the scale is plotted as a function of mean $J / \psi$ curvature, we observe an upward trend, that is, scale increasing with increasing curvature, indicating an excess of ionizing material in the detector simulation. By globally scaling the ionizing material in the simulation by a factor of 0.98 , the dependence of scale on curvature is removed and the plot becomes flat, see Figure 7.4. 


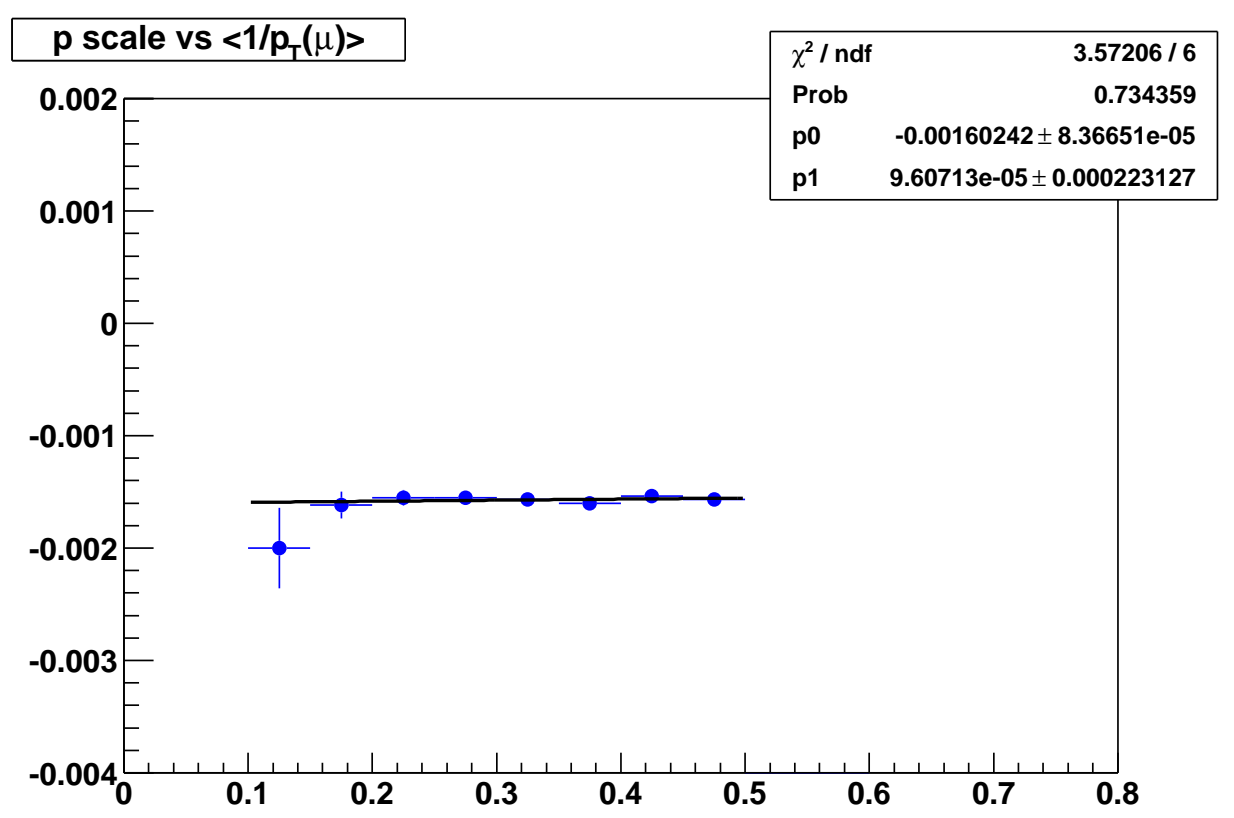

Figure 7.4: $\Delta p / p$ vs. mean curvature for $J / \psi$ events after material scaling. The scale is made independent of mean curvature by globally scaling the ionizing material in the detector simulation by 0.98 . We extrapolate the scale to zero curvature to obtain the final momentum scale from $J / \psi \rightarrow \mu \mu$.

Since we are interested in the scale at high $p_{T}$ (small curvature) we take the $y$-intercept of the plot of scale versus curvature as the momentum scale from $J / \psi \rightarrow \mu \mu$. This value is

$$
\frac{\Delta p}{p}(J / \psi)=(-1.60 \pm 0.08) \times 10^{-3}
$$

where the uncertainty is purely statistical.

\subsubsection{Systematic Uncertainties on the $J / \psi$ Scale}

There are several sources of systematic uncertainty on the measurement of the $J / \psi$ momentum scale, the largest of which is from QED radiation off the final state di-muon pair. 
To estimate this, we vary the $Q$-scale used when evaluating the form-factor (equation 7.1) to minimize the $\chi^{2}$ value of the inclusive $J / \psi$ invariant mass fit. We observe a change in momentum scale of $0.20 \times 10^{-3}$ associated with this variation. As a cross-check of this procedure, we measure the change in scale as we vary the lower boundary of the fit to the invariant mass peak. This gives an uncertainty of $0.08 \times 10^{-3}$.

The second largest systematic uncertainty comes from non-uniformity of the magnetic field. We investigate this by looking at the scale dependence on $\cot \theta$, as shown in Figure 7.5. We fit cubic and quadratic functions to this dependence and then use these to correct the data. We observe at most a change in momentum scale of $0.10 \times 10^{-3}$ after the corrections are applied, so we take this as the systematic uncertainty.

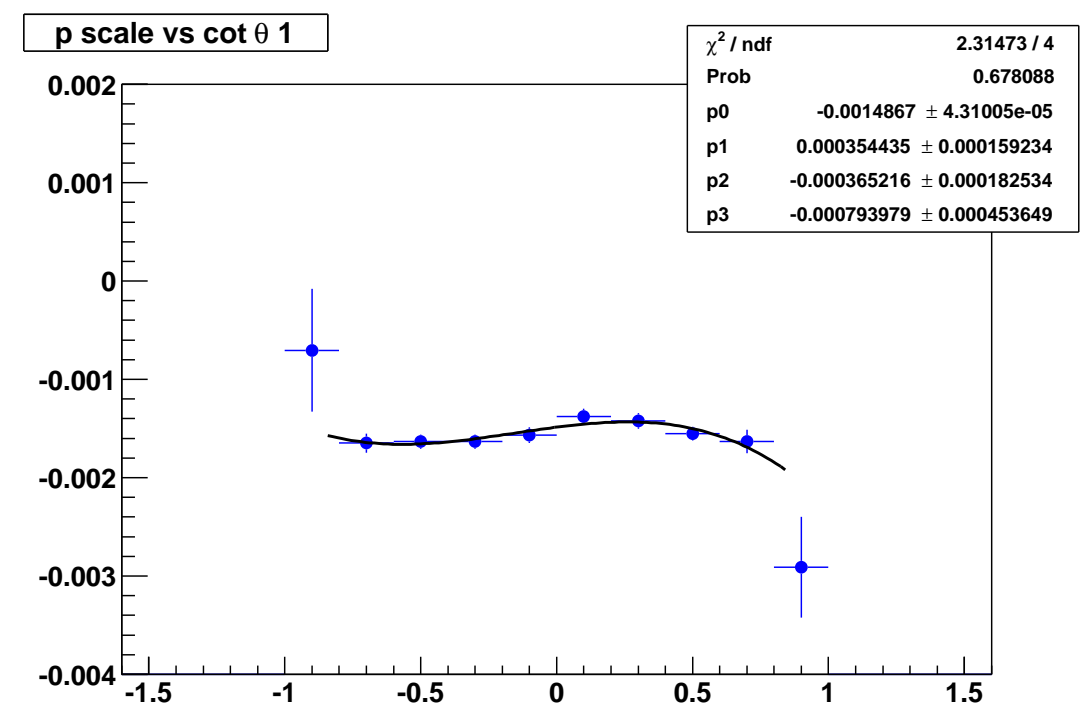

Figure 7.5: $\Delta p / p$ vs. $\cot \theta$ before corrections. This particular plot shows a cubic function fit to the data in the region $-0.1<\cot \theta<0.1$. The variation in momentum scale associated with applying this function to the data is taken as a systematic uncertainty.

All other systematic uncertainties, with brief descriptions of how they are estimated, are included in the Table 7.1.

As a check of any $\phi$ dependent systematic uncertainty on the momentum scale, we 


\begin{tabular}{|c|l|c|}
\hline Systematic Source & \multicolumn{1}{|c|}{ Evaluation Method } & Value $\left(\times 10^{-3}\right)$ \\
\hline QED & $\begin{array}{l}\text { Vary } Q \text { value in form factor to minimize } \\
\chi^{2} \text { of inclusive fit }\end{array}$ & 0.20 \\
\hline Muon Energy Loss & $\begin{array}{l}\text { Vary material scale over } \pm 1 \sigma \text { changes } \\
\text { in slope of scale vs. } 1 /<p_{T}^{\mu}>\text { plot }\end{array}$ & 0.01 \\
\hline Kinematics & Vary muon $p_{T}$ cuts by $\pm 100 \mathrm{MeV} / c$ & 0.04 \\
\hline Backgrounds & $\begin{array}{l}\text { Vary normalization to minimize } \chi^{2} \text { of } \\
\text { COT Resolution }\end{array}$ & 0.03 \\
\hline Non-uniformity of B field & Correct data to make scale vs. cot $\theta$ flat & 0.10 \\
\hline Misalignment & Vary cot $\theta$ and curvature corrections & 0.05 \\
\hline Fitting Procedure/Window & Use fit windows $20 \%$ larger/smaller & 0.05 \\
\hline
\end{tabular}

Table 7.1: Systematic uncertainties on the $J / \psi \rightarrow \mu \mu$ momentum scale. The quadrature sum of these uncertainties gives $0.25 \times 10^{-3}$ as the overall systematic uncertainty on the momentum scale from $J / \psi \rightarrow \mu \mu$.

plot the scale as a function of $\phi$ of the muon track, see Figure 7.6. The fluctuations observed are consistent with the overall uncertainty quoted on the scale.

\subsection{COT Momentum Scale from $\Upsilon(1 S) \rightarrow \mu \mu$}

As mentioned before, a benefit of using the $\Upsilon$ sample to measure the COT momentum scale is that it allows us to investigate any systematic uncertainties associated with beamconstraining tracks. In addition, with a mass of $9460.30 \pm 0.26 \mathrm{MeV} / c^{2}$ [7], it provides a measurement of the scale at an intermediate point between the lighter $J / \psi$ and the heavier $W$ and $Z$ bosons. 


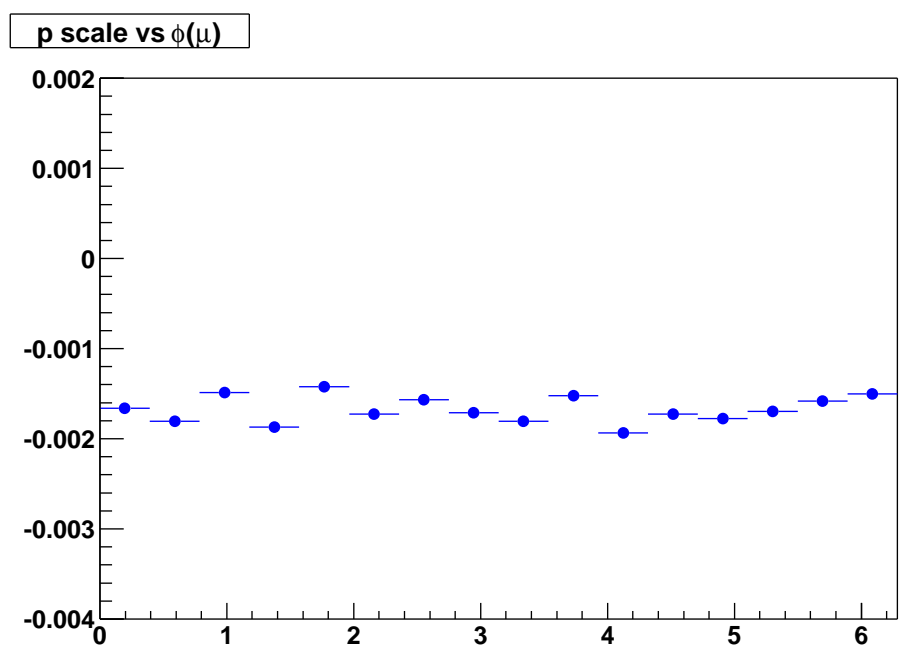

Figure 7.6: $\Delta p / p$ vs. muon $\phi$.

\subsubsection{Fitting templates}

Using a procedure exactly analogous to the generation of $J / \psi$ events, we use PYTHIA to generate $10^{6} \Upsilon(1 S) \rightarrow \mu \mu$ events. QED is included via a form factor and we apply the kinematic tuning obtained from the $J / \psi$ generation. To model the background we fit the $\Upsilon$ invariant mass region with three Gaussians and a linear background, see Figure 7.7. We include this background in the templates.

\subsection{2 $\Upsilon$ Analysis}

We apply the 0.98 material scaling and the curvature corrections (equations 7.3 and 7.4) obtained from the $J / \psi$ data. We determine the COT hit resolution and extract the momentum scale from a fit to the $1 S$ invariant mass peak. For the non beam-constrained sample we use a fit range of $9.27-9.59 \mathrm{GeV} / c^{2}$ and for the beam-constrained sample we use $9.30-9.56 \mathrm{GeV} / c^{2}$, the difference resulting from better resolution for the beamconstrained sample, see Figure 7.8 . 


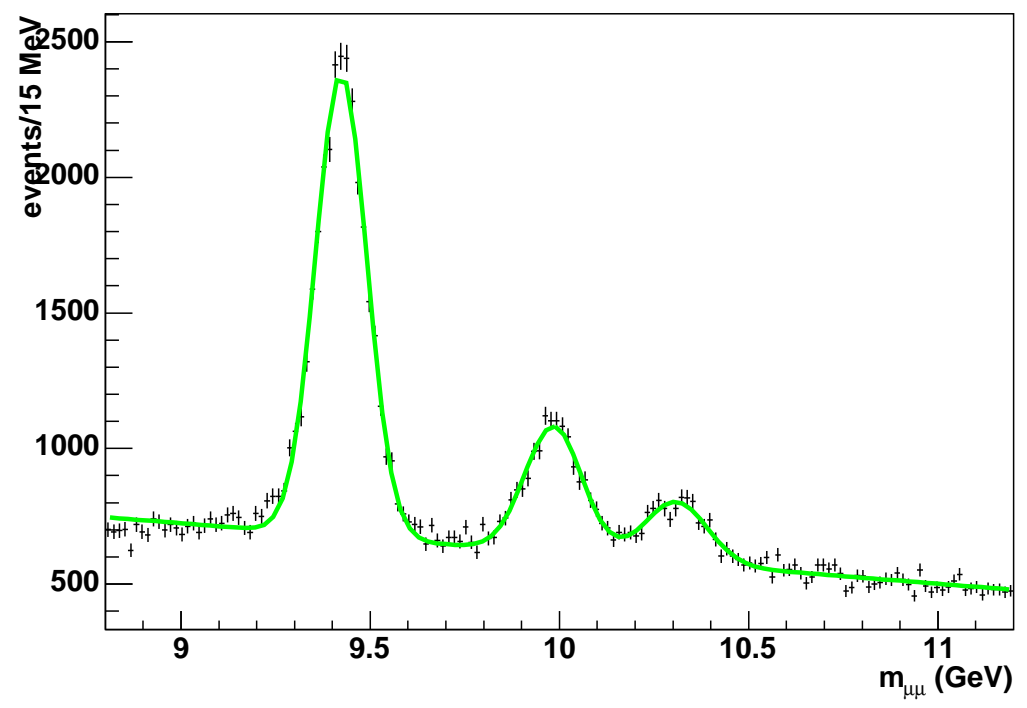

Figure 7.7: Upsilon $1 S, 2 S$, and $3 S$ resonances. We fit the data with three Gaussians and a linear background function. From this we extract the background to be included in the fitting templates.
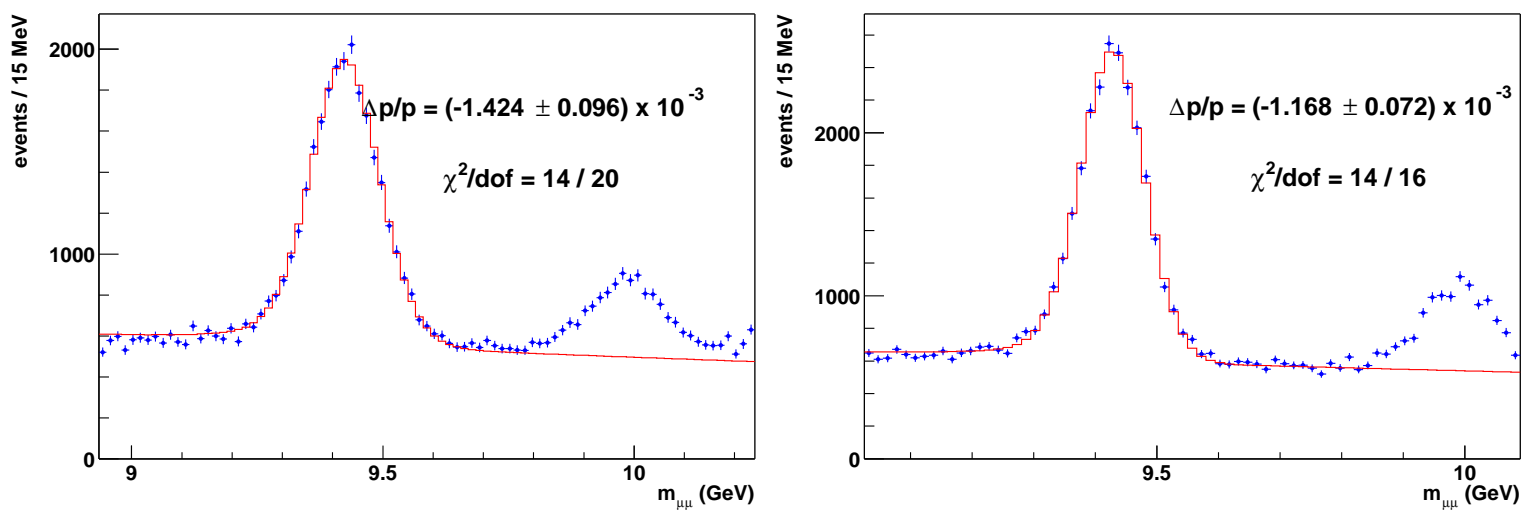

Figure 7.8: Momentum scales obtained from $\Upsilon(1 S) \rightarrow \mu \mu$ events. The left plot shows the fit to the $1 S$ invariant mass peak using non beam-constrained COT tracks. The right plot shows the same for beam-constrained tracks. 
For the non beam-constrained case we obtain a scale of,

$$
\frac{\Delta p}{p}\left(\Upsilon_{\text {non BC }}\right)=(-1.42 \pm 0.10) \times 10^{-3}
$$

For the beam constrained case we obtain a scale of,

$$
\frac{\Delta p}{p}\left(\Upsilon_{\mathrm{BC}}\right)=(-1.17 \pm 0.07) \times 10^{-3}
$$

The difference between beam-constrained and non beam-constrained momentum scales is statistically significant if we do not consider the systematic uncertainties on the scales. However, if we include systematic uncertainties we find the scales are in reasonable agreement. Nevertheless, to account for any potential discrepancy due to beam-constraining, we include an additional systematic uncertainty in the combined $\Upsilon$ scale of half the difference between the beam-constrained and non beam-constrained scales. As a further check, we computed these scales using data samples that were processed with an updated version of the COT alignment. We obtained values of $\frac{\Delta p}{p}\left(\Upsilon_{\text {non BC }}\right)=(-1.38 \pm 0.10) \times 10^{-3}$ and $\frac{\Delta p}{p}\left(\Upsilon_{\mathrm{BC}}\right)=(-1.26 \pm 0.07) \times 10^{-3}$, which are (i) consistent with the quoted scales above, and (ii) now consistent with each other.

\subsubsection{Systematic Uncertainties on the $\Upsilon$ Scale}

Systematic uncertainties on the $\Upsilon$ scale are estimated in a similar fashion to those for the $J / \psi$ scale, with the largest uncertainty once again coming from QED and the difference between the beam-constrained and non beam-constrained scales, see Table 7.2.

\subsection{Combined Momentum Scale}

We use a Best Linear Unbiased Estimator (BLUE) [45] method to find the combined momentum scale and calculate the overall uncertainty. First the $\Upsilon$ scales (beam con- 


\begin{tabular}{|c|l|c|}
\hline Systematic Source & \multicolumn{1}{|c|}{ Evaluation Method } & Value $\left(\times 10^{-3}\right)$ \\
\hline QED & Vary fit range in invariant mass fit & 0.13 \\
\hline Muon Energy Loss & $\begin{array}{l}\text { Vary material scale over } \pm 1 \sigma \text { changes } \\
\text { in slope of scale vs. } 1 /<p_{T}^{\mu}>\text { plot }\end{array}$ & 0.05 \\
\hline Kinematics & Vary muon $p_{T}$ cuts by $\pm 100 \mathrm{MeV}$ & 0.02 \\
\hline Backgrounds & Vary slope of background fit & 0.01 \\
\hline COT Resolution & Vary hit resolution by $\pm 10 \mu$ m & 0.03 \\
\hline Non-uniformity of B field & Apply correction obtained from $J / \psi$ & 0.12 \\
\hline Misalignment & Sample & 0.03 \\
\hline Fitting Procedure/Window & Use fit windows $20 \%$ larger/smaller & 0.02 \\
\hline$\Upsilon_{\text {BC vs. } \Upsilon_{\text {non BC }}}$ & Half the difference between scales & 0.13 \\
\hline Correlated statistical & Common statistical uncertainty be- & 0.07 \\
\hline
\end{tabular}

Table 7.2: Systematic uncertainties on the $\Upsilon(1 S) \rightarrow \mu \mu$ momentum scale. Note that these uncertainties apply to both the non beam-constrained and beam-constrained scales. We take the common statistical uncertainty between the two as $100 \%$ correlated and include a systematic uncertainty of half the difference between the beam-constrained and non beam-constrained scales. The quadrature sum of all the uncertainties gives an overall systematic uncertainty of $0.23 \times 10^{-3}$ on the combined $\Upsilon(1 S)$ scale. 
strained and non-beam constrained) are combined. This value is then combined with the $J / \psi$ scale to obtain the final momentum scale of,

$$
\frac{\Delta p}{p}=\left(-1.45 \pm 0.05_{\text {stat. }} \pm 0.21_{\text {syst. }}\right) \times 10^{-3}=(-1.45 \pm 0.22) \times 10^{-3}
$$

The uncertainty on this final scale translates into an uncertainty of $18 \mathrm{MeV} / c^{2}$ on the $W$ mass. Combining this with the curvature correction uncertainty of $10 \mathrm{MeV} / c^{2}$ discussed in section 6.2, we obtain an overall momentum scale uncertainty of $21 \mathrm{MeV} / c^{2}$.

\subsection{Momentum Scale Cross-check with $Z \rightarrow \mu \mu$}

As a cross-check, we apply the combined momentum scale to the $Z \rightarrow \mu \mu$ sample and construct the invariant mass. Recall that we model QED radiation of the di-muon pair and include Drell-Yan and $Z / \gamma$ interference terms in the final templates as described in sections 4.1.5 and 4.2.2. We fit the data to Monte Carlo in the region, $83<M_{Z}<99$ $\mathrm{GeV} / c^{2}$, see Figure 7.9.

We find a $Z$ mass of,

$$
\begin{aligned}
M_{Z} & =\left(91.181 \pm 0.043_{\text {stat. }} \pm 0.022_{\text {syst. }}\right) \mathrm{GeV} / c^{2} \\
& =(91.181 \pm 0.051) \mathrm{GeV} / c^{2}
\end{aligned}
$$

where the systematic uncertainty comes from the measured momentum scale extrapolated to the $Z$ mass and the $10 \mathrm{MeV} / c^{2}$ curvature correction systematic. The measured $Z$ mass is consistent with the world average value of $(91.188 \pm 0.002) \mathrm{GeV} / c^{2}[7]$.

We also determine the COT hit resolution to use for the $W \rightarrow \mu \nu$ events from the width of the $Z$ peak. An iterative process to minimize the $\chi^{2}$ of the invariant mass fit yields a value of $(200 \pm 15) \mu \mathrm{m}$. The uncertainty is obtained from a change of one unit of $\chi^{2}$ in the fit. This translates to a $11 \mathrm{MeV} / c^{2}$ systematic uncertainty on the $W$ mass. 


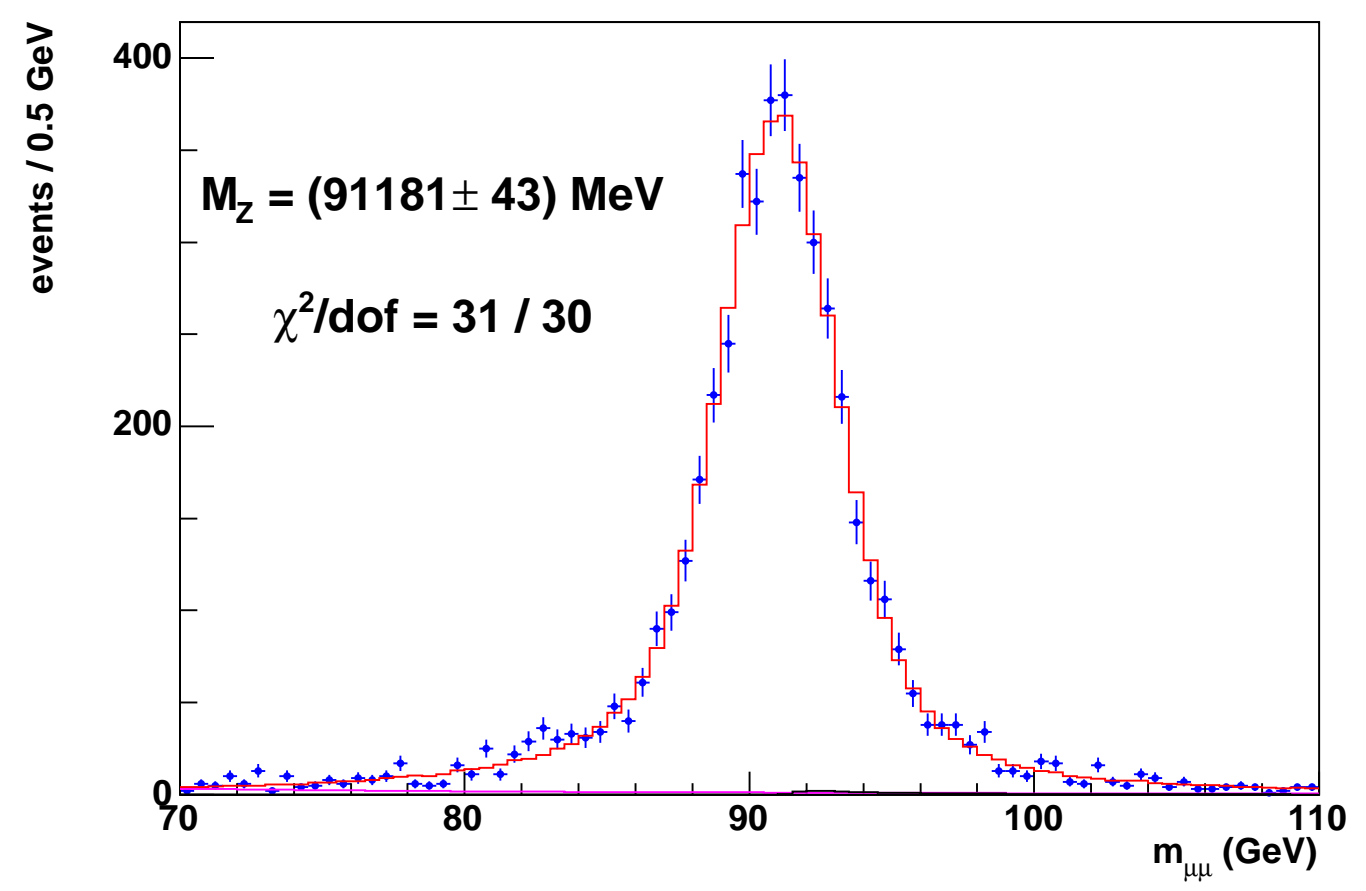

Figure 7.9: $Z \rightarrow \mu \mu$ invariant mass fit. The combined momentum scale of $-1.45 \times 10^{-3}$ has been applied. The fit value is consistent with the world average value of $(91.188 \pm$ $0.002) \mathrm{GeV} / c^{2}$. 


\section{Chapter 8}

\section{Results}

The final stage of the measurement is extracting the $W$ mass from a fit to the muon $p_{T}$ spectrum. This is done using a specially designed fitter that is described below. As a cross-check, we also determine the $W$ mass from fits to the $\mathbb{H}_{T}$ and $M_{T}^{W}$ spectra. The correlations between the three fit methods are investigated using Monte Carlo.

\subsection{Fitting Data to Monte Carlo}

The final stage of the measurement of the $W$ mass is fitting the data to the templates. As mentioned in section 6 we run generator level Monte Carlo at 80 and $81 \mathrm{GeV} / c^{2}$ through the fast simulation. A linear extrapolation is performed between these two fully simulated templates to produce a series of templates suitable for fitting to the data, see Figure 8.1.

These templates are input to a fitter that adds background contributions and normalizes the Monte Carlo to the data in the fit region. We then compute the Poisson probability of the data in each bin using the expected number of events as given by the Monte Carlo templates. A likelihood function is calculated as the product of the Poisson probabilities over all bins in the fit region. The best fit $W$ mass is selected as the value that maximizes the likelihood function. 


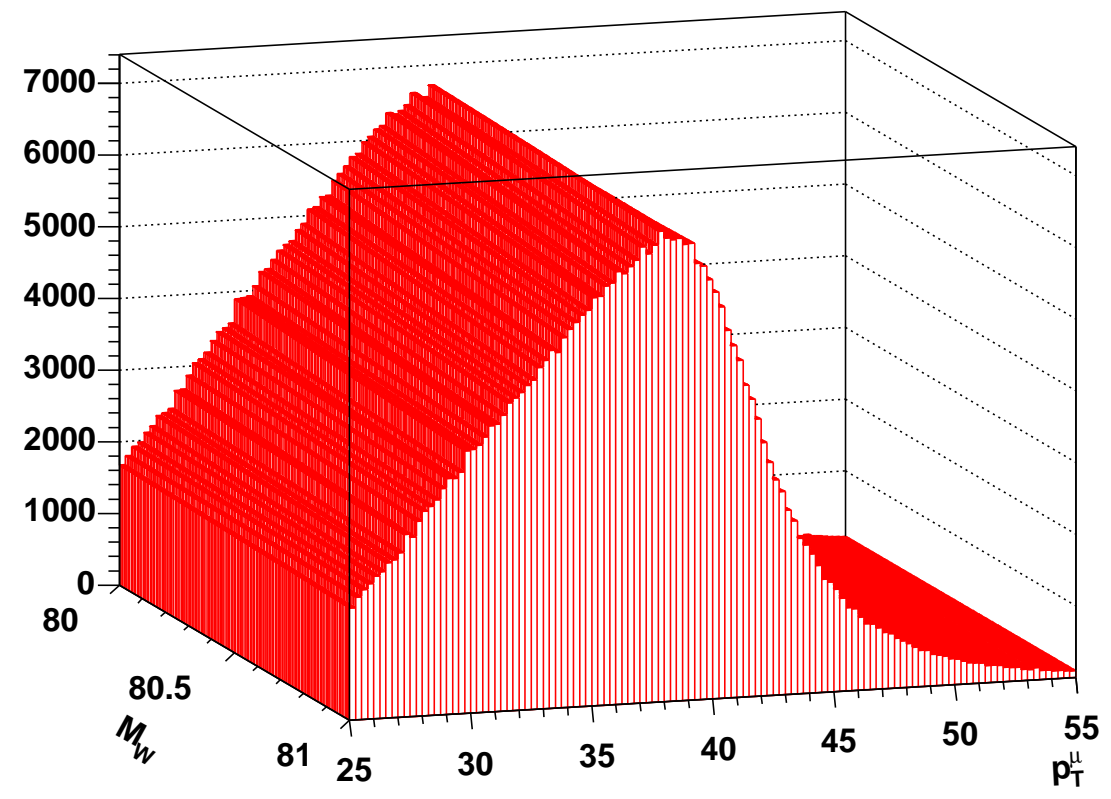

Figure 8.1: Illustration of the $p_{T}^{\mu}$ templates generated by the fast simulation.

\subsection{Correlations between Mass Fits}

We investigate correlations between the $p_{T}^{\mu}, \mathscr{E}_{T}$, and $M_{T}^{W}$ fits using large Monte Carlo samples run through the fast simulation. We generate $60 \times 10^{6} \mathrm{~W} \rightarrow \mu \nu$ events with $M_{W}=80.450 \mathrm{GeV} / c^{2}$. We split the sample into subsamples with the same statistics as the $W$ data. We run each of these subsamples through the fast simulation and record the best fit mass from each of the kinematic fits: $p_{T}^{\mu}, M_{T}^{W}$, and $\mathscr{H}_{T}$. For each pair of fits we compute a correlation factor,

$$
r_{x y}=\frac{\sum_{i}^{N} x_{i} y_{i}-\sum_{i}^{N} x_{i} \sum_{i}^{N} y_{i}}{N \sigma_{x} \sigma_{y}}
$$

where $x_{i}$ is the $i^{\text {th }}$ value of fit type $x, y_{i}$ is the $i^{t h}$ value of fit type $y, \sigma_{x}$ and $\sigma_{y}$ are the statistical uncertainties on $x$ and $y$, and $N$ is the number of subsamples.

Scatter plots of the results are shown in Figure 8.2. The measured correlations are 

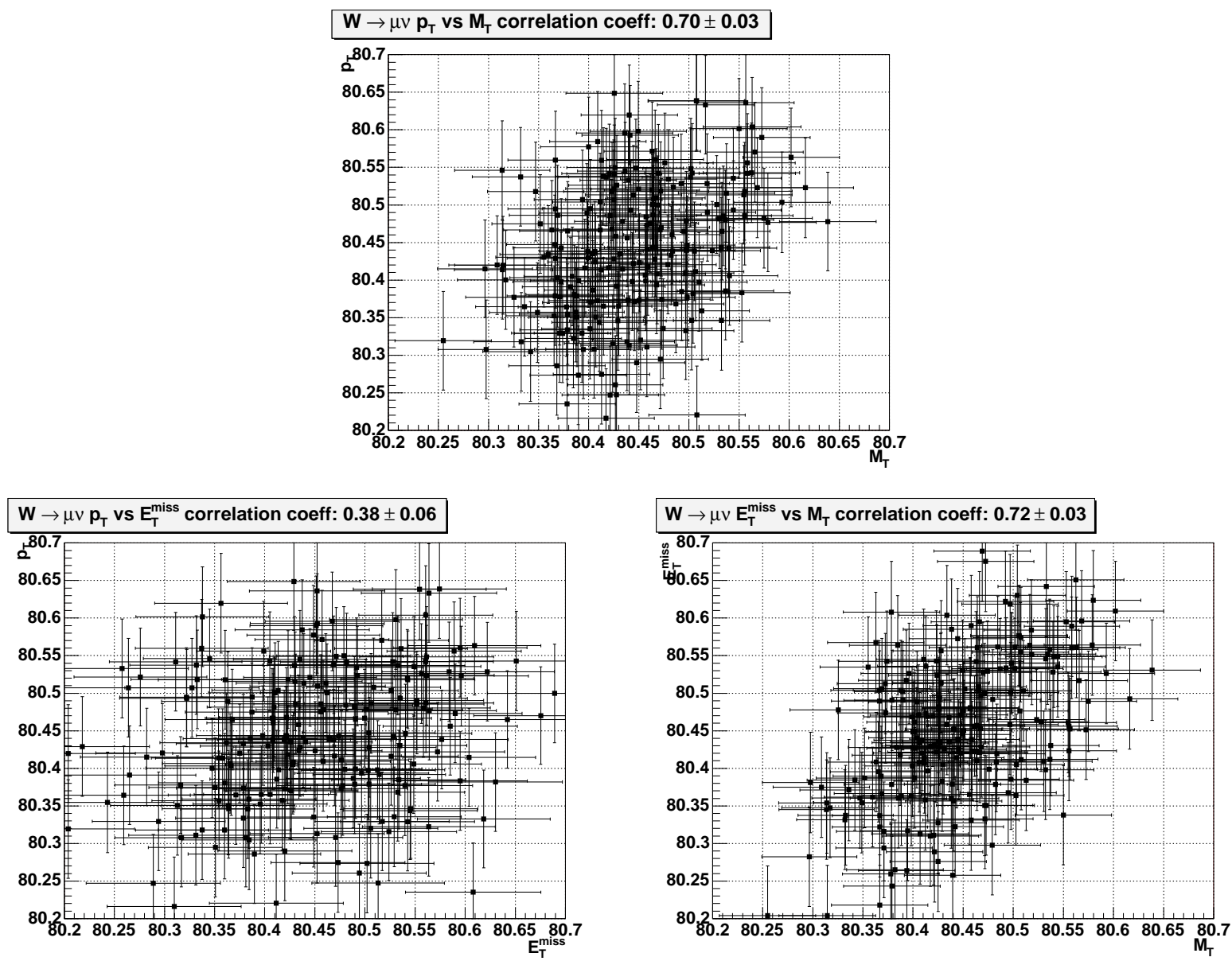

Figure 8.2: Correlations between $W$ mass fit types.

shown in Table 8.1.

\begin{tabular}{|c|c|}
\hline Fit Types & Correlation Factor $(r)$ \\
\hline$p_{T}^{\mu}$ vs. $\not \not_{T}$ & $0.38 \pm 0.06$ \\
\hline$M_{T}^{W}$ vs. $\not \not_{T}$ & $0.72 \pm 0.03$ \\
\hline$p_{T}^{\mu}$ vs. $M_{T}^{W}$ & $0.70 \pm 0.03$ \\
\hline
\end{tabular}

Table 8.1: Measured correlations between $W$ mass fit types in the muon channel. We can see $M_{T}^{W}$ is fairly strongly correlated with both $p_{T}^{\mu}$ and $\not_{T}$ since both these quantities are used in computing the transverse mass. $p_{T}^{\mu}$ and $\mathscr{H}_{T}$ are much less correlated. 


\subsection{Systematic Uncertainties on the $p_{T}$ Fit}

The systematic uncertainties contributing to the $W$ mass measurement in the muon channel are presented in Table 8.2.

\begin{tabular}{|c|c|}
\hline Systematic Uncertainty & Value $\left(\mathrm{MeV} / c^{2}\right)$ \\
\hline Momentum Scale & 21 \\
\hline COT Momentum Resolution & 11 \\
\hline Recoil Scale & 5 \\
\hline Recoil Resolution & 7 \\
\hline$u_{\|}$Efficiency & 5 \\
\hline Backgrounds & 20 \\
\hline$p_{T}^{W}$ Model & 27 \\
\hline QED & 17 \\
\hline \hline$\Gamma_{W}$ & 18 \\
\hline Tarton Distribution Functions & 12 \\
\hline \hline
\end{tabular}

Table 8.2: Systematic uncertainties on the $W$ mass for the $p_{T}^{\mu}$ method.

One class of uncertainties comes from the $W$ production model, which includes QED, QCD, and PDFs. The QED uncertainty of $17 \mathrm{MeV} / \mathrm{c}^{2}$ comes from a fitter systematic uncertainty, the bias introduced by ISR and interference terms, varying the $\delta_{s}$ parameter in WGRAD (photon energy cut), and gauging the impact of a second photon. These results are summarized in Table 4.5. In section 4.2 .4 we vary the phenomenological parameters $g_{2}$ and $g_{3}$ in RESBOS to estimate the systematic uncertainty associated with QCD. We find an uncertainty of $27 \mathrm{MeV} / c^{2}$. PDFs (section 4.4) are accounted for using a set of error PDFs, giving $18 \mathrm{MeV} / c^{2}$.

The event selection process introduces a $5 \mathrm{MeV} / \mathrm{c}^{2}$ uncertainty via the muon ID 
efficiency as a function of $u_{\|}$(section 5.1.4).

The recoil model parameters for the response function, $R$, and the resolution are discussed in section 6.4.2. Varying the parameters $a$ and $b$ in the response function by their uncertainties gives a systematic uncertainty of $5 \mathrm{MeV} / c^{2}$. Varying the recoil resolution parameters $s_{h a d}$ and $N$ within their uncertainties gives a systematic uncertainty of $7 \mathrm{MeV} / c^{2}$.

The systematic uncertainties arising from the calibration of the COT tracker come from the uncertainty on the combined momentum scale, with the dominant uncertainty coming from QED radiation. We obtain a $21 \mathrm{MeV} / \mathrm{c}^{2}$ systematic, which includes the uncertainty from curvature corrections, presented in sections 6.2 and 7.3. We use the width of the $Z \rightarrow \mu \mu$ distribution to constrain the COT hit resolution (section 7.4). This variation in COT hit resolution results in an $11 \mathrm{MeV} / c^{2}$ uncertainty on the $W$ mass.

Backgrounds are evaluated using a variety of techniques. The $Z \rightarrow \mu \mu$ and $W \rightarrow \tau \nu$ backgrounds are modelled with Monte Carlo. A QCD rich subsample is obtained from data and then is used in conjunction with $W \mathrm{MC}$ or $Z$ data to estimate the QCD background. The kaon background is evaluated from the track $\chi^{2}$ distribution and crosschecked with the $\Delta \phi(u, l)$ distribution. The cosmic ray background is investigated via the $t_{0}$ distribution. All backgrounds are shown in Figure 5.6 and contribute a total systematic uncertainty of $20 \mathrm{MeV} / c^{2}$.

In addition to the above, we attribute a systematic uncertainty from the $W$ width. In all our calculations we use a value of $(2.12 \pm 0.07) \mathrm{GeV}$. Translating the $70 \mathrm{MeV}$ uncertainty to the $W$ mass gives a an uncertainty of $12 \mathrm{MeV} / c^{2}$.

\subsection{Result}

We measure the $W$ mass from a fit to the $p_{T}^{\mu}$ spectrum in the range $30<p_{T}^{\mu}<45 \mathrm{GeV} / c$. We find a value of, 


$$
\begin{aligned}
M_{W} & =\left(80.316 \pm 0.066_{\text {stat. }} \pm 0.051_{\text {syst. }}\right) \mathrm{GeV} / c^{2} \\
& =(80.316 \pm 0.083) \mathrm{GeV} / c^{2}
\end{aligned}
$$

We can see in Figure 8.3 there is good agreement between data and Monte Carlo. Figure 8.4 shows the $\log$ likelihood is a smooth function with a well-defined minimum. Figure 8.5 shows the bin-by-bin difference between data and Monte Carlo divided by the uncertainty on the data (signed $\chi$ statistic).

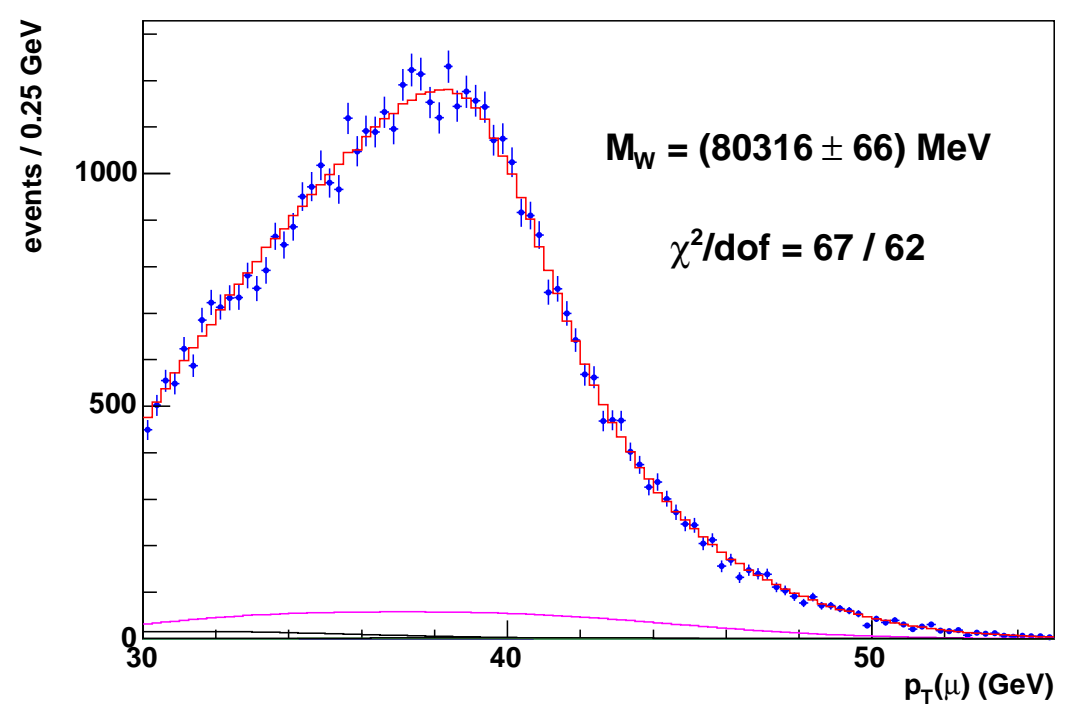

Figure 8.3: $W$ mass from a fit to the $p_{T}^{\mu}$ spectrum.

\subsection{Cross-checks}

We perform several cross-checks of the measurement. The first of these is made by measuring the mass using the $\mathbb{H}_{T}$ and $M_{T}^{W}$ methods.

The $\not_{T}$ fit gives a result of $(80.453 \pm 0.101) \mathrm{GeV} / c^{2}$ and the $M_{T}^{W}$ fit gives $(80.370 \pm$ 0.075) GeV/ $/ c^{2}$. Given the measured correlations we use a BLUE method [45] to combine 


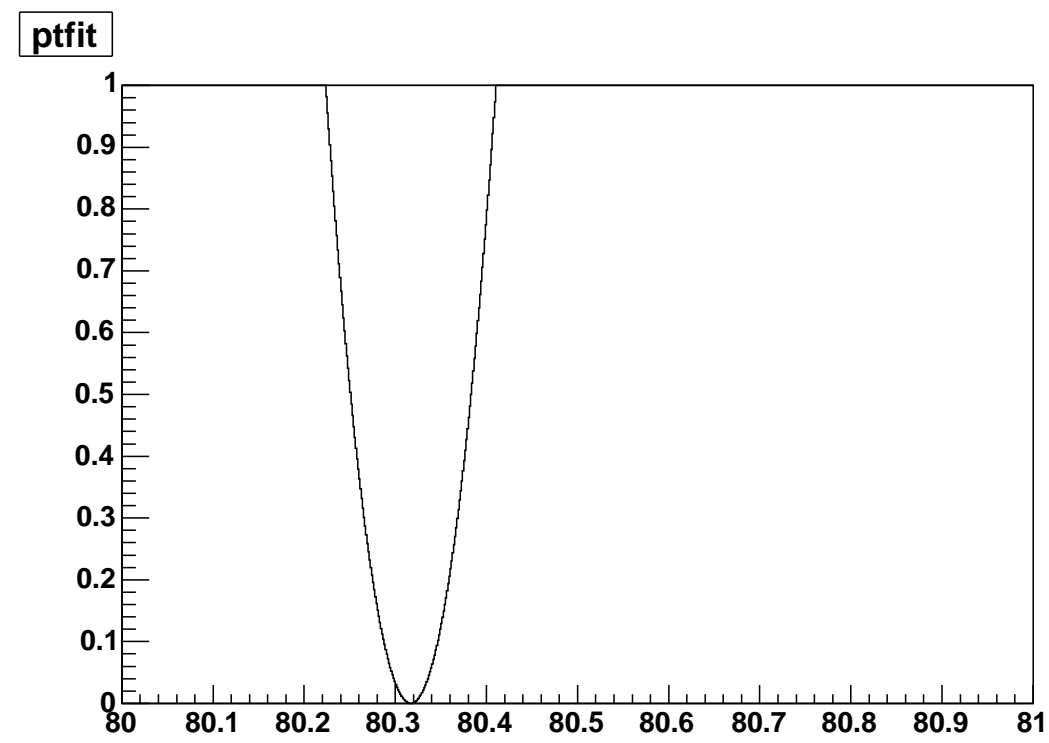

Figure 8.4: The $\log$ likelihood of the fit to the $p_{T}^{\mu}$ spectrum. The minimum of this function defines the best-fit $W$ mass.

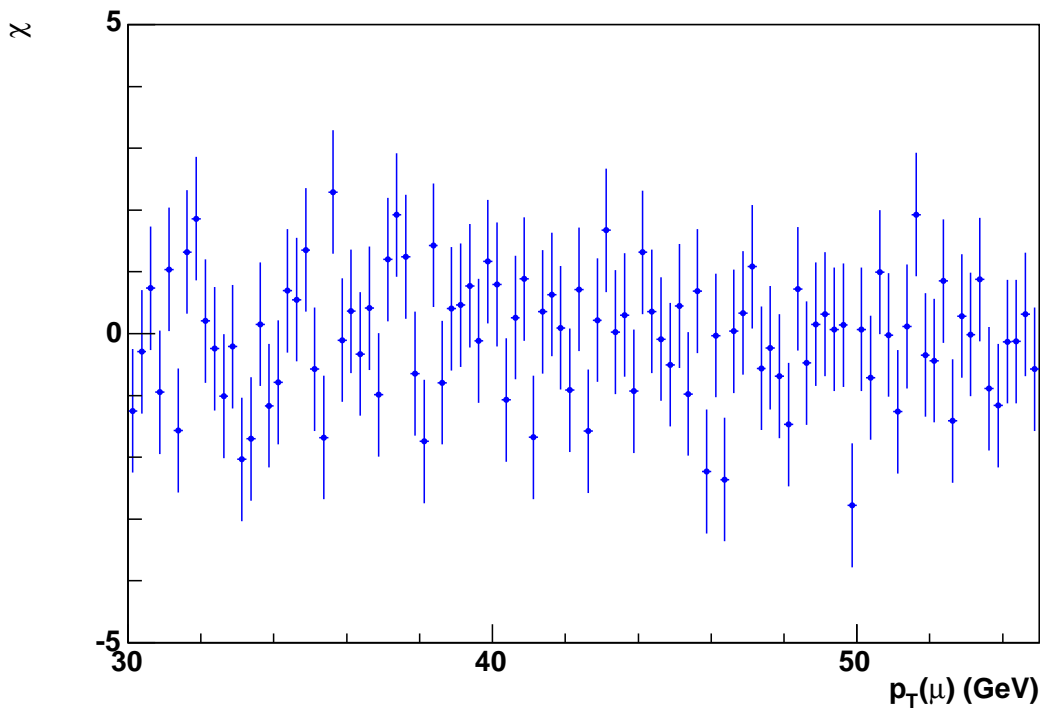

Figure 8.5: Signed $\chi$ plot of the fit to the $p_{T}^{\mu}$ spectrum. There are no apparent discrepancies between data and Monte Carlo. 
the results and compute their self-consistency. We find a $\chi^{2} /$ dof value of $3 / 2$, indicating the results are consistent with each other.

We carry out further cross-checks of the measurement. We compute the mass for various subsamples: $W^{+}$vs. $W^{-}$, lower half of the COT vs. upper half, and early data vs. late data. The results are shown in Table 8.3. The $W^{+}$vs. $W^{-}$split shows a $\sim 2 \sigma$ discrepancy indicating that the curvature corrections may need further tuning. However, it should be noted that since the inclusive $W$ sample contains both the positive and negative charges, the final fit value is quite robust under any changes in the curvature corrections. The other two subsamples show no statistically significant discrepancies.

\begin{tabular}{|c|c|}
\hline Cross check & Result $\left(\mathrm{MeV} / c^{2}\right)$ \\
\hline$M_{W}\left(\mu^{+}\right)-M_{W}\left(\mu^{-}\right)$ & $294 \pm 136$ \\
\hline$M_{W}\left(\phi_{\mu}>0\right)-M_{W}\left(\phi_{\mu}<0\right)$ & $-17 \pm 133$ \\
\hline$M_{W}($ early $)-M_{W}($ late $)$ & $-74 \pm 136$ \\
\hline
\end{tabular}

Table 8.3: Cross-checks of the $W$ mass measurement. The upper half of the COT corresponds to $\phi_{\mu}>0$. Early vs. late data corresponds to splitting the data taking period into two roughly equal segments.

\subsection{Impact}

This measurement of the $W$ mass is consistent with the world average value of $(80.392 \pm$ $0.029) \mathrm{GeV} / c^{2}$. When this measurement is included, the world average value becomes $(80.384 \pm 0.027) \mathrm{GeV} / c^{2}$. See Table 8.4 for a comparison with previous $W$ mass measurements.

To illustrate the significance of this result we can consider what the uncertainties on the muon $p_{T}$ mass measurement might be with increasingly larger data samples. We can divide all systematic uncertainties into two categories, one that scales with statistics 


\begin{tabular}{|c|c|}
\hline Experiment & Mass $\left(\mathrm{MeV} / c^{2}\right)$ \\
\hline L3 & $80270 \pm 55$ \\
DELPHI & $80336 \pm 67$ \\
OPAL & $80416 \pm 53$ \\
ALEPH & $80440 \pm 51$ \\
CDF Run I & $80433 \pm 79$ \\
D0 Run I & $80483 \pm 84$ \\
This measurement & $80316 \pm 83$ \\
\hline \hline LEP Average & $80376 \pm 33$ \\
Tevatron Run I Average & $80452 \pm 59$ \\
\hline World Average & $80392 \pm 29$ \\
\hline New World Average & $80384 \pm 27$ \\
\hline
\end{tabular}

Table 8.4: Current world's best $W$ mass measurements. The value obtained in this thesis is $0.9 \sigma$ lower than the current world average value. The "new world average" is the current world average including this measurement.

and one that doesn't. Those that don't scale with statistics are from the $W$ production model: QED, $p_{T}^{W}$ model, and PDFs. Combining the uncertainties from these sources in quadrature gives $37 \mathrm{MeV} / \mathrm{c}^{2}$. The remaining systematic uncertainties combine to give $35 \mathrm{MeV} / c^{2}$, which combined with the statistical uncertainty of $66 \mathrm{MeV} / c^{2}$ gives $75 \mathrm{MeV} / c^{2}$. The statistical uncertainty as well as the non-production model systematic uncertainties scale with $1 / \sqrt{N}$, where $N$ is the data sample size. With a $2 \mathrm{fb}^{-1}$ sample (10 times the current sample size) the $75 \mathrm{MeV} / c^{2}$ would decrease to $24 \mathrm{MeV} / c^{2}$; with $8 \mathrm{fb}^{-1}$, this would become $12 \mathrm{MeV} / \mathrm{c}^{2}$. This would leave the $37 \mathrm{MeV} / c^{2}$ production model uncertainty as the dominant uncertainty if it is not improved upon. We can see this is a good time to focus our efforts on improving these methods, especially since the quality of transverse mass measurement will degrade due to decreased $\mathscr{E}_{T}$ resolution. 
Already there is potential for improvement in the near future: there are several recent calculations of a second photon (the dominant QED uncertainty) as well as a program that calculates the combined effect of NLO QCD with soft-gluon resummation and a single photon in the final state (RESBOS-A). In addition, we could use a larger sample of $Z$ bosons to help constrain the $g$ parameters of RESBOS. These improvements could reduce the production model systematic uncertainty by as much as a factor of two in the next few years. If we consider this coupled with the $2 \mathrm{fb}^{-1}$ data sample, the total uncertainty on the muon channel measurement would be $30 \mathrm{MeV} / c^{2}$, already less than the LEP average.

\subsection{Conclusion}

Using $191 \mathrm{pb}^{-1}$ of data collected by the CDF II detector, we measure the $W$ mass

from a fit to the $p_{T}$ spectrum of the muon in $W$ decay to be $\left(80.316 \pm 0.066_{\text {stat. }} \pm\right.$ $\left.0.051_{\text {syst. }}\right) \mathrm{GeV} / c^{2}=(80.316 \pm 0.083) \mathrm{GeV} / c^{2}$. Many of the uncertainties investigated in this thesis will be relevant for future measurements of the $W$ mass since the $p_{T}$ fit is likely to be the primary method for obtaining the $W$ mass. 


\section{Bibliography}

[1] M. Gaillard et al. The Standard Model of Particle Physics. Rev. Mod. Phys., 71:96, 1999.

[2] G. Arnison et al. Experimental observation of isolated large transverse energy electrons with associated missing energy at $\hat{s}=540 \mathrm{GeV}$. Phys. Lett. B, 122:103, 1983.

[3] The LEP Electroweak Working Group. http://lepewwg.web.cern.ch/LEPEWWG/ plots/summer2006, 2006.

[4] S. Glashow. Partial-symmetries of weak interactions. Nucl. Phys., 22:579, 1961.

[5] S. Weinberg. A model of leptons. Phys. Rev. Lett., 19:1264, 1967.

[6] M. Gell-Mann. A schematic model of baryons and mesons. Phys. Lett., 8:214, 1964.

[7] W.-M. Yao et al. Review of Particle Physics. J. Phys. G, 33:1, 2006.

[8] P. Higgs. Broken Symmetries and the Masses of Gauge Bosons. Phys. Rev. Lett., 13:508, 1964.

[9] The ALEPH, DELPHI, L3, OPAL, SLD Collaborations, the LEP Electroweak Working Group, the SLD Electroweak and Heavy Flavour Groups. Precision Electroweak Measurements on the Z Resonance. hep-ex/0509008, page 302, 2005.

[10] S. M. Thurman-Keup et al. W Boson Physics at Hadron Colliders. Rev. Mod. Phys., 73:267, 2001. 
[11] E. Mirkes. Angular decay distribution of leptons from W-bosons at NLO in hadronic collisions. Nucl. Phys. B, 387:3, 1992.

[12] J. Collins and D. Soper. Angular distribution of dileptons in high-energy hadron collisions. Phys. Rev. D, 16:2219, 1977.

[13] V. Buge et al. Prospects for the precision measurement of the $W$ mass with the CMS detector at the LHC. CMS Note, 061, 2006.

[14] The CDF II Detector Technical Design Report. FERMILAB-Pub-96/390E, 1996.

[15] C. Balazs and C.-P. Yuan. Soft gluon effects on lepton pairs at hadron colliders. Phys. Rev. D, 56:5558, 1997.

[16] U. Baur, S. Keller, and D. Wackeroth. Electroweak radiative corrections to $W$ boson production in hadronic collisions. Phys. Rev. D, 59:013002, 1998.

[17] T. Affolder et al. Measurement of the $W$ Boson Mass with the Collider Detector at Fermilab. Phys. Rev. D., 64:052001, 2001.

[18] F.A. Berends et al. QED Radiative Corrections And Radiative Decays Of The Intermediate Weak Bosons Produced In Proton - Anti-Proton Collisions. Z. Phys. $C, 27: 155,1985$.

[19] E. Barberio et al. PHOTOS: A Universal Monte Carlo for QED radiative corrections in decays. Comp. Phys. Commun, 66:115, 1991.

[20] G.P. Lepage. A new algorithm for adaptive multidimensional integration. J. Comp. Phys., 27:192, 1978.

[21] M. Lancaster and D. Waters. A Monte Carlo Program for $W$ Production with Electroweak Radiative Corrections. CDF Note, 5240, 2000.

[22] F.A. Berends and R. Kleiss. Z. Phys. C, 27:365, 1985. 
[23] U. Baur. Private Comunication.

[24] C.M. Carloni Calame et al. Higher-order QED corrections to W-boson mass determination at hadron colliders. Phys. Rev. D., 69:037301, 2004.

[25] A. Gibson et al. An Event Generator for Drell-Yan Dilepton Production with Electroweak Radiative Corrections. CDF Note, 6334, 2003.

[26] I. Hinchliffe. The Papageno Partonic Monte Carlo Program. LBL-34372.

[27] J. Strologas and Steven Errede. Study of the angular coefficients and corresponding helicity cross sections of the $\mathrm{W}$ boson in hadron collisions. Phys. Rev. D, 73:8, 2005.

[28] W.T. Giele et al. Higher-order corrections to jet cross sections in hadron colliders. Nucl. Phys. B, 403:633, 1993.

[29] B. Abbott et al. Measurement of the $W$ boson mass. Phys. Rev. D, 58:092003, 1998.

[30] M. Lancaster. A Recoil Model for the Run 1B $W$ Mass Analysis. CDF Note, 4048, 1997.

[31] F. Landry et al. Fermilab Tevatron run-1 Z boson data and the Collins-SoperSterman resummation formalism. Phys. Rev. D, 67:073016, 2003.

[32] F. Landry et al. New fits for the non-perturbative parameters in the CSS resummation formalism. Phys. Rev. D, 63:013004, 2000.

[33] C. P. Yuan and Q. H. Cao. Combined Effect of QCD Resummation and QED Radiative Correction to W Boson Observables at the Tevatron. Phys. Rev. Lett, 93:042001, 2004.

[34] J. Pumplin et al. New generation of parton distributions with uncertainties from global QCD analysis. JHEP, 7:12, 2002. 
[35] A. Martin et al. MRST partons and uncertainties. hep-ph/0307262, 2003.

[36] T. Sjostrand et al. PYTHIA 6.2 Physics and Manual. hep-ph/0108264, 2001.

[37] A. Kotwal et al. Identification of Cosmic Rays using Drift Chamber Hit Timing. Nucl. Instrum. Methods A, 506:110, 2003.

[38] A. Kotwal et al. Fast Detector Simulation for the $W$ Mass Measurement. CDF Note, $7573,2005$.

[39] K. Rinnert. A Fast-Access Material Map for the SVX II/ISL Silicon Tracking Volume at CDF 2. CDF Note, 5846, 2004.

[40] S. Agostinelli et al. GEANT 4 - a simulation toolkit. Nucl. Instrum. Methods A, 506:250, 2003.

[41] S. Lai et al. Measurement of the beam width at CDF. CDF Note, 7270, 2004.

[42] D. Attwood et al. The scattering of muons in low Z materials. hep-ex/0512005, 2006.

[43] A. Kotwal and C. Hays. COT Internal Alignment using Cosmic Rays. COT Workshop, U. of Chicago, 2003.

[44] A. Kotwal et al. Measurement of the $W$ Boson Mass in Run 2. CDF Note, 7104, 2004.

[45] L. Lyons et al. Combining correlated estimates of a single physical quantity. Nucl. Instrum. Methods A, 270:110, 1988. 